\title{
CITEseq analysis of non-small-cell lung cancer lesions reveals an axis of immune cell activation associated with tumor antigen load and TP53 mutations
}

\author{
Andrew M. Leader ${ }^{1,2,3}$, John A. Grout ${ }^{1,2,3}$, Christie Chang ${ }^{1,4}$, Barbara Maier ${ }^{1,2,3}$, Alexandra \\ Tabachnikova ${ }^{1,2,3}$, Laura Walker ${ }^{1,4}$, Alona Lansky ${ }^{1,2,3}$, Jessica LeBerichel ${ }^{1,2,3}$, Naussica Malissen ${ }^{1,2,3}$, \\ Melanie Davila, ${ }^{1,4}$,Jerome Martin ${ }^{1,2,3,5}$, Giuliana Magri ${ }^{1,2,3,}$, Kevin Tuballes ${ }^{1,4}$, Zhen Zhao ${ }^{6}$, Francesca \\ Petralia $^{7,8}$, Robert Samstein ${ }^{1,2,3,9}$, Natalie Roy D'Amore ${ }^{10}$, Gavin Thurston ${ }^{11}$, Alice Kamphorst ${ }^{1,2,3}$, Andrea

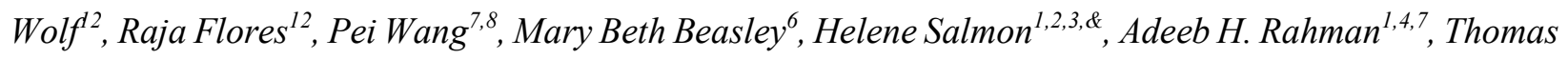 \\ U. Marron $^{1,2,13}$, Ephraim Kenigsberg ${ }^{1,7,8, \$}$, Miriam Merad $^{1,2,3,4, \$}$
}

\footnotetext{
${ }^{1}$ The Precision Immunology Institute, Icahn School of Medicine at Mount Sinai, New York, NY, USA.

${ }^{2}$ The Tisch Cancer Institute, Icahn School of Medicine at Mount Sinai, New York, NY, USA.

${ }^{3}$ Department of Oncological Sciences, Icahn School of Medicine at Mount Sinai, New York, NY, USA.

${ }^{4}$ Human Immune Monitoring Center, Icahn School of Medicine at Mount Sinai, New York, NY, USA.

${ }^{5}$ Nantes Université, CHU Nantes, Inserm, Centre de Recherche en Transplantation et Immunologie, UMR 1064 , F-44000 Nantes, France : CHU Nantes, Nantes Université, Laboratoire d'Immunologie, F-44000 Nantes, France

${ }^{6}$ Department of Pathology, Icahn School of Medicine at Mount Sinai, New York, NY, USA.

${ }^{7}$ Department of Genetics and Genomic Sciences, Icahn School of Medicine at Mount Sinai, New York, NY, USA.

${ }^{8}$ Icahn Institute for Genomics and Multiscale Biology, Icahn School of Medicine at Mount Sinai, New York, NY, USA.

${ }^{9}$ Department of Radiation Oncology, Icahn School of Medicine at Mount Sinai, New York, NY, USA.

${ }^{10}$ Immuno-oncology Drug Discovery Unit, Millennium Pharmaceuticals, Inc. a wholly owned subsidiary of Takeda Pharmaceutical Company Limited.

${ }^{11}$ Department of Oncology \& Angiogenesis, Regeneron Pharmaceuticals Inc., Tarrytown, NY, 10591, USA

${ }^{12}$ Department of Thoracic Surgery, Icahn School of Medicine at Mount Sinai, New York, NY, USA.

${ }^{13}$ Division of Hematology/Oncology, Icahn School of Medicine at Mount Sinai, New York, NY, USA.
}

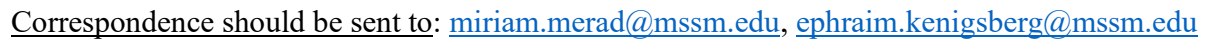

\author{
${ }^{\$}$ Authors contributed equally to this work \\ \#Present address: Program for Inflammatory and Cardiovascular Disorders, Institut Hospital del Mar d'Investigacions Mèdiques \\ (IMIM), 08003 Barcelona, Spain \\ \&Present address: INSERM U932, Institut Curie, 26 rue d’Ulm, 75005 Paris, France
}




\section{SUMMARY}

2 Immunotherapy is becoming a mainstay in the treatment of NSCLC. While tumor mutational

3 burden (TMB) has been shown to correlate with response to immunotherapy, little is known about

4 the relation of the baseline immune response with the tumor genotype. Here, we profiled 35 early

5 stage NSCLC lesions using multiscale single cell sequencing. Unsupervised clustering identified

6 in a subset of patients a key cellular module consisting of PDCD1+CXCL13+ activated T cells,

$7 \quad \mathrm{IgG}+$ plasma cells, and $S P P 1+$ macrophages, referred to as the lung cancer activation module

8 (LCAM $\left.^{\text {hi }}\right)$. Transcriptional data from two NSCLC cohorts confirmed a subset of patients with

9 LCAM $^{\text {hi }}$ enrichment, which was independent of overall immune cell content. The LCAM ${ }^{\text {hi }}$ module

10 strongly correlated with TMB, expression of cancer testis antigens, and with TP53 mutations in

11 smokers and non-smokers. These data establish LCAM as a key mode of immune cell activation 12 associated with high tumor antigen load and driver mutations. 


\section{INTRODUCTION}

15 Lung cancer is the most common cause of cancer-related death ${ }^{1}$, and the most common subgroup

16 of lung cancer is non-small cell lung cancer (NSCLC) ${ }^{2}$. In recent years, immune checkpoint

17 blockade (ICB) targeting the PD-1/PD-L1 axis has become first-line therapy for a majority of

18 patients with metastatic and locally advanced disease ${ }^{3}$. Though ICB studies have achieved

19 improved overall survival, fewer than half of patients achieve significant clinical benefit, though

20 still may experience physical and financial toxicity. Biomarkers are lacking to determine optimal

21 treatment regimens for patients, as our understanding of tumor-associated immune phenotypes and

22 immune correlates of response to ICB remains incomplete.

While multiple studies have used single-cell assays to profile NSCLC tumor-infiltrating

24 immune cells in comparison to patient-matched, non-involved lung (nLung) $)^{4,5}$, blood ${ }^{6}$, or both bo, $^{7,8}$,

25 or characterized tumor-infiltrating lymphocytes (TIL) $)^{9-12}$, we continue to lack a comprehensive

26 understanding of how immune cell phenotypes vary across patients. In particular, it remains

27 unclear which immune cell populations and phenotypes are associated with robust, tumor-directed

28 T cell responses and response to ICB, and how these features are connected to tumor-cell intrinsic

29 characteristics such as tumor mutational burden $(\mathrm{TMB})^{13,14}$. A deeper analysis is further required

30 for uncovering the cell types and states associated with immunostimulatory versus

31 immunoregulatory presentation of tumor-associated antigens, as well as parsing the tumor-related

32 effects on tissue-resident and migratory innate cell types. Attempts to integrate these data across

33 the innate and adaptive arms of the immune system are of crucial importance to optimizing rational

34 design of immunotherapies. Furthermore, while response to ICB has been associated with specific

35 patient groups, individual driver mutations, the degree of immune infiltrate, and TMB, the complex

36 interplay between these factors remains poorly understood. 
Here, we sought to define the molecular immune states induced in the tumor

38 microenvironment by profiling an expanded patient cohort compared to previous related studies ${ }^{5,6}$

39 via multiscale single-cell analyses. We integrated the results of single-cell RNA sequencing

40 (scRNAseq) of immune cells with cellular indexing of transcriptomes and epitopes by sequencing

41 (CITEseq $)^{15}$, a method allowing for combined scRNAseq and multiplexed single-cell surface

42 protein measurement. To further elucidate the TCR landscape across T cell phenotypes, we

43 analyzed these results together with joint scRNAseq/TCRseq. We revealed a pattern of inter-tumor

44 variability involving innate and adaptive immune responses which we validated in two bulk RNA

45 datasets, allowing us to detect an association with TMB and tumor driver mutations.

\section{$47 \quad$ RESULTS}

49 Integrative analyses unify phenotypic mappings across substrates and datasets. To probe

50 transcriptional states of immune cells in the lung cancer microenvironment, we set out to profile

51 cells from a cohort of untreated, early-stage NSCLC patients undergoing resection with curative

52 intent (Figure 1A). The cohort was diverse with respect to age, smoking status, sex, and

53 histological subtype (Figure 1B). We generated three datasets integrating antibody profiling of

54 surface marker proteins using CITEseq ${ }^{15}$, scRNAseq, and TCRseq with single cell resolution

55 (Tables S1-S3). We performed CITEseq on matched tumor and non-involved lung (nLung) tissues

56 from 7 patients, in addition to performing scRNAseq of matched tumor and nLung in 28 additional

57 patients. Finally, to expand on our annotation of T cell clusters based on the distribution of clonally

58 expanded populations, we performed paired single-cell TCRseq and scRNAseq on T cells isolated

59 from 3 patients. 
ScRNA profiles in tumor and nLung were clustered together using a batch-aware algorithm

61 we recently developed in order to combine data across patients, while accounting for batch-specific

62 background noise ${ }^{16}$ (Figures $1 \mathrm{C}$ and S1A-C). To develop a general gene-expression model of

63 clusters representing different cell types and states, we relied only on 19 nLung and 22 tumor

64 samples processed with 10X Chromium 3' V2 chemistry. We then used this model to classify cells

65 from additional samples processed with different protocols or from different datasets showing

66 similar transcriptional profiles. (Figure S1B-D, methods). The RNA-based clustering identified

6749 immune clusters within 6 compartments including subsets of T cells, B cells, plasma cells, mast

68 cells, plasmacytoid dendritic cells (pDC), and mononuclear phagocytes (MNP) consisting of

69 macrophages (MФ), monocytes, putative monocyte-derived dendritic cells (MoDC), and

70 conventional dendritic cells (cDC; Figure 1C and S1E, F). Overall, 377,549 single cells from 35

71 tumors and 32 matched nLung samples from patients at Mount Sinai were classified into 6

72 compartments and 30 annotated transcriptional states. CITEseq data further confirmed cell

73 identities using well-established protein markers (Figure 1D). For example, annotation of pDC

74 was based on expression of transcripts associated with this lineage (LILRA4, IRF8; Figure 1C) and

75 high expression of known population-defining surface markers (CD123; Figure 1D). While cluster

76 frequencies varied widely among patients, clusters mapped between 590 and 23812 cells, and all

77 clusters included cells from multiple patients (Figure 1E and S1F).

We first compared the variability of samples from different regions within individual

79 tumors to the variability between different patients' tumors with respect to immune cell type

80 composition. To do this, we examined samples from a study that analyzed three regions per tumor

81 in 8 patients $^{5}$, mapping cells to the clusters produced with our expanded dataset. Clustering the

82 samples by correlation of cell type frequencies among immune cells demonstrated that samples 
83 almost always clustered by patient (Figure S1G), and similarly, the Euclidean distances between

84 patient-matched samples of different tumor regions was strongly reduced compared to the

85 distances between samples from different patients (Figure S1H). Therefore, while the total level

86 of immune content may still vary regionally in and around tumors ${ }^{17}$, these analyses demonstrated

87 that inter-tumor differences drive lung tumor immune variability in terms of the phenotypic

88 makeup of the immune cells that are present.

To understand whether the immune changes between tumor and nLung were distinct across

90 patients or, alternatively, globally similar, we estimated the immune diversity within tumor and

91 nLung using the Euclidean distances between the log-transformed cluster-frequencies. This

92 analysis indicated that nLung samples were significantly more homogeneous (Figure 1F; "nLung-

93 nLung distances") than tumor samples ("Tumor-Tumor distances"; $t=8.3 ; p<2.2 \mathrm{e}-16$ ). We further

94 compared distances among nLung and among tumor to the distances between nLung and tumor.

95 This analysis showed that the diversity between independent (unmatched) tumor and nLung

96 samples was larger than the diversity within tumor samples $(\mathrm{t}=19.6 ; \mathrm{p}<2.2 \mathrm{e}-16)$ and $\mathrm{nLung}$

97 samples $(\mathrm{t}=24.6 ; \mathrm{p}<2.2 \mathrm{e}-16)$, suggesting that immune landscapes within the TME were

98 significantly changed compared to non-involved tissues, and that most tumors harbored many

99 conserved changes (Figure 1E-F).

100 We next sought to test if the differences between nLung and tumor could be observed in 101 an independent cohort. The cell type frequencies of 8 matched tumor-nLung pairs described in 102 ref. ${ }^{5}$ indeed validated the distinct microenvironments we observed in our cohort (Figure $1 \mathrm{G}$ ). This 103 result demonstrated that the observed tumor signatures were robust and reproducible, encouraging 104 us to further study the transcriptional states within it. 

derived DC. We next investigated the heterogeneity with the myeloid compartment, given that different myeloid cells have various important roles in generating or inhibiting tumor directed immune responses, including antigen presentation, T cell co-stimulation, and shaping the cytokine milieu within the $\mathrm{TME}^{13}$. We identified conventional DC1 (cDC1) expressing IRF8, WDFY4, and

111 CLEC9A transcripts (Figure 2A) as well as CD141 and CD26 surface markers (Figure 2B), and 112 cDC2 expressing high $C D 1 C$ and $F C E R 1 A$ transcripts as well as CD1c and CD5 protein. We also 113 detected a DC cluster expressing FSCN1 and CCR7 transcripts and elevated HLADR, CD86, PD$114 \mathrm{~L} 1$, and CD40 surface protein which we described as mature DC enriched in regulatory molecules 115 (mregDC) in great detail elsewhere ${ }^{18}$; in this study we found mregDC were correlated with tumor116 antigen uptake and thus help define antigen-charged $\mathrm{DC}^{18}$. This phenotype was also consistent 117 with an activated DC phenotype detected in lung and liver tumors by others ${ }^{6,19}$. We furthermore 118 identified clusters that expressed cDC2 markers such as CD1c and CLEC10A, but also expressed 119 high levels of monocyte and MФ genes including $S 100 A 8, S 100 A 9, C 1 Q A$, and $C 1 Q B$, lacked 120 surface expression of the pre-DC surface marker $C D 5^{20,21}$, and exhibited increased expression of 121 CD11b and CD14 (Figure 2A, B); we annotated such clusters as MoDC. Importantly, MoDC were 122 distinct from $\mathrm{M} \Phi$ based on higher levels of CD1c surface protein in addition to their upregulation 123 of the DC2-like transcriptional signature (Figure 1C, clusters 52, 29, and 30). Overall, MoDC were 124 the most prevalent DC subtype and were increased in tumors compared to nLung, whereas 125 mregDC were the rarest (Figure $2 \mathrm{C}$ and S2A-C). As we had seen previously ${ }^{7}$, the fraction of cDC1 126 were strongly reduced in tumors (Figure 2C).

Since the activation profile of mregDC is crucial for inducing tumor directed $\mathrm{T}$ cell 128 responses $^{18}$, we examined the mregDC distribution in tumors by multiplexed 
129 immunohistochemical consecutive staining on a single slide (MICSSS ${ }^{22}$. We stained for DC-

130 LAMP and PD-L1, as the transcripts of these genes (LAMP3 and CD274, respectively) were highly

131 enriched in the mregDC cluster (Figure 2D). We found that mregDC expressing DC-LAMP and

132 PD-L1 accumulated in tertiary lymphoid structures (TLS) in close proximity to T cells (Figure

133 2E). CD3-negative areas of TLS, which are putatively analogous to lymph node B cell zones, were

134 frequently populated by MYH11+ follicular dendritic cells ${ }^{23}$, a stromal cell type commonly found 135 in B cell zones (Figure 2F).

To better understand the relationship between MoDC and other MNP, we searched for

137 genes that were mutually exclusive among CD14 monocytes, cDC2, and MФ (Figure S2D, Table

138 S4). Scoring MoDC using these gene lists in comparison with other MNP populations revealed

139 that MoDC were distinct from MФ and CD14+ monocytes. Ordering cells within each of these

140 compartments by the expression of these distinct monocyte- and cDC2 gene programs

141 demonstrated anticorrelation of these gene sets among MoDC but not cDC2 (Figure 2G;

142 rho $=-0.33, \mathrm{p}<2.2 \mathrm{e}-16 ;$ rho $=0.016, \mathrm{p}=0.29$, respectively), demonstrating that MoDC inhabited a

143 phenotypic spectrum between monocytes and cDC2-like cells. While some M $\Phi$ genes were

144 expressed in MoDC higher than in $\mathrm{cDC} 2$ cells, MoDC were distinct from MФ based on higher

145 cDC2 gene expression and lower MФ gene expression (score distributions are detailed in Figure

146 S2E).

To further uncover transcriptional programs that were variable among MoDC and cDC

148 without relying on specific cell classifications, we analyzed the covariance structure of variable

149 genes among all DC. This approach resulted in distinct sets of co-expressed genes (gene

150 "modules") that varied together across cells, independent of cluster assignments (Figure S2F, G,

151 Table S5). Gene module analysis across the DC compartment revealed upregulation in tumors of 
152 multiple modules that were mainly restricted to MoDC and DC2 (Figure S2H, I). The gene

153 modules most upregulated in tumors compared to nlung included genes associated with glycolysis

$154(\bmod 39)$ and cell cycle $(\bmod 38)$, which were mainly expressed in MoDC cluster 52 (Figure S2G-

155 I). Frequent upregulation of many monocyte- or M $\Phi$-like modules $(7,3,4,6,5,37,10)$ was

156 consistent with a higher frequency of MoDC compared to cDC in tumors.

157 We also identified a cDC2 module (mod34) which was enriched in tumor lesions compared 158 to nLung (Figure S2G, H) and included $C D 1 A$ and $C D 207$. These genes mark the lesional cells of 159 Langerhans cell histiocytosis ( $\mathrm{LCH}$ ), a myeloid inflammatory condition driven by enhanced ERK 160 activation ${ }^{24}$; we therefore referred to this module as "LCH-like". LCH cells produce many 161 inflammatory cytokines that promote the accumulation of Tregs and activated $\mathrm{T}$ cells in $\mathrm{LCH}$ 162 lesions ${ }^{25}$. Interestingly, IL22RA2, encoding the IL22 decoy receptor IL22-BP, was also included 163 in this module (Table S5). IL22 modulates epithelial cell growth and plays a role in tissue 164 protection through modulation of tissue inflammation and in promoting tumor growth through 165 induction of tissue repair ${ }^{26}$. Expression of the IL22 receptor (IL22RA1), meanwhile, negatively 166 correlated with survival in KRAS-mutated lung cancer lesions ${ }^{27}$. These genes were mainly induced 167 in the bona fide cDC2 cluster, but were also upregulated in MoDC (Figure 2H). Probing DC 168 expression in an independent scRNAseq dataset of NSCLC immune cells ${ }^{5}$ confirmed upregulation 169 of these genes in tumor associated DC transcriptomes (Figure S2J).

\section{Tumors are dominated by monocyte-derived МФ that are distinct from alveolar МФ.}

172 While previous studies have demonstrated phenotypic differences between M $\Phi$ populating nLung 173 versus tumors ${ }^{5,7}$, they have been limited in their ability to parse specific $М \Phi$ subpopulations with 174 potentially distinct ontogeny and function. Our data showed remarkable heterogeneity within the 
$175 \mathrm{M} \Phi$ compartment as demonstrated by the varying expression of classical marker genes among

176 clusters (Figures $3 \mathrm{~A}$ and S3A). This level of resolution allowed the identification of alveolar M $\Phi$

177 (AMФ) clusters expressing SERPINA1 and PPARG and a cluster expressing genes consistent with

178 interstitial MФ (IMФ), which thus far have only been defined to a limited extent in humans ${ }^{28,29}$. In

179 contrast to AMФ that self-renew locally independent of blood precursors ${ }^{30}$, IM $\Phi$ are thought to be

180 maintained by circulating monocyte pools even in steady state, albeit at lower rates of turnover

181 than in settings of overt inflammation. IM $\Phi$ lacked $P P A R G$ and expressed $M A F$ family

182 transcription factors, MERTK, CSF1R, LYVE1, and CX3CR1 $1^{31}$. CD14+ and CD16+ monocytes

183 were defined by the expression of CD14 or FCGR3A respectively and the lack of MФ markers

184 MRC1, VSIG4, and SIGLEC1. Other MФ clusters expressed genes such as MAFB, CEBPD,

$185 F C G R 2 B$, and CSF1R, which are indicative of monocyte origin and shared by monocytes and

$186 \mathrm{IM} \Phi$; therefore, these clusters were annotated as МоМФ. A remaining population of MФ

187 expressed genes consistent with primary granule formation (AZU1, ELANE, CTSG) but distinct

188 from bone marrow progenitors due to lack of $M P O$, and also lacked elevation of neutrophil marker

189 genes $^{6} C S F 3 R, L R G 1, F F A R 4$, and $V A S P$ compared to other myeloid cells (Figure S3A). This

190 cluster was referred to as $A Z U 1+\mathrm{M} \Phi$.

Using a CITEseq panel of established immune surface markers, we validated the

192 transcription-based cluster annotations and associated new surface markers with the MФ

193 subpopulations. For example, we found that CD10, not previously appreciated as a M $\Phi$ marker,

194 could distinguish AMФ from other lung myeloid populations (Figures 3B and S3B). This staining

195 was consistent with RNA expression patterns (Figure S3A) and was verified by

196 immunohistochemical staining (IHC) of airspace-residing AM $\Phi$ in nLung (Figure S3C). MoMФ

197 expressed higher levels of CD11c and CD14 than other MФ populations, whereas IMФ were 


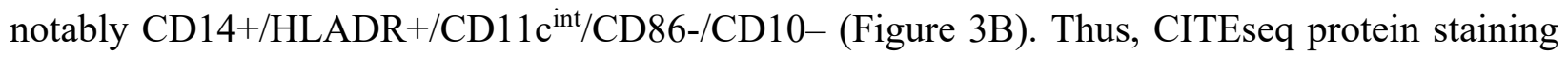
confirmed the main $\mathrm{M} \Phi$ subpopulations identified by the transcriptional classification and defined potential sorting strategies (Figure S3B). cell states reflecting, for example, interferon response (modules 32 and 19), heat shock genes

206 (module 49) cell cycle (module 42), HLA class-II expression (module 28), and glycolysis (module

210 receptor antagonist $I L 1 R N$, and module 47 consisting of genes indicating a glycolytically active

211 state (GAPDH, ENO1, LDHA, ALDOA, TPI1), and lower levels than other M $\Phi$ of C1Q and HLA-

212 class-II transcripts (Figures 3C and S3E, G). MoMФ-III clusters were enriched in module 24

213 (including TREM2 and LILRB4) and module 25 (including APOE and GPNMB). MoM

214 MoMФ-IV were less distinctive than the other MoMac subtypes, but each comprised their own

215 unique gene expression patterns. For example, MoMac-IV expressed the highest levels of module

21627 which included CTSS, $C F D$, and $A L D H 1 A 1$ and also expressed some genes otherwise confined

217 to АМФ (module 36), whereas MoMФ-I was enriched in module 20 (including chemokine ligands

218 CCL13 and CCL2) while MoMac IV was not. Together, these analyses identify multiple tumor

219 MoMФ phenotypes with distinct metabolic and immunomodulatory gene programs that are

220 enriched in the tumor milieu and likely contribute to defining the tumor microenvironment. 
Gene set scores based on mutually exclusive, differentially expressed genes among CD14+

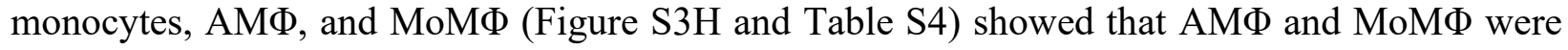
each distinct from CD14+ monocytes (Figure 3D) but that MoMФ expressed a gradient of the

224 CD14+ monocyte score (Figure 3E). Analysis of the gene expression patterns of hundreds of genes within the scores supported the general trends (Figure 3F). MoMФ clusters were also distinct from the IMФ cluster based on many transcripts and surface proteins (Figure 3A, B), although some $\operatorname{MoM} \Phi$, especially those that were the most distant from AMФ, shared some IM $\Phi$ genes such as CSF1R, FOLR2, and MERTK (Figures 3A, F and S3A). strongly depleted from tumors and IMФ frequencies were unchanged (Figure 3G). Monocyte frequencies were also decreased, possibly reflecting their differentiation to MoMФ or MoDC.

232 Given that individual MoMФ-subsets changed between nLung and tumor to different extents, we

233 asked how these differences related to the underlying phenotypic heterogeneity within the MoM $\Phi$ 234 compartment, beyond signatures revealed by module analysis. Selecting for a set of highly 235 expressed transcripts encoding secreted factors demonstrated strong differences between MNP 236 subsets (Figure 3H). MoMФ-II, the most tumor-enriched subset, expressed the highest levels of 237 inflammatory cytokines $T N F$ and $I L 6$, transcripts encoding the pleiotropic factor SPP1, a broad 238 collection of matrix metallopeptidases $M M P-7,-9$, and -12 , as well as CCR2/5 ligands $C C L-2,-8$, 239 and -7. By comparison, other MoMФ populations expressed less distinct secretory profiles. 240 Multiple MNP populations expressed the CXCR3-ligand chemotactic factors CXCL-9,-10,-11 241 including $\mathrm{MoM} \Phi, \mathrm{MoDC}$, and mregDC, while these ligands were distinctly absent from $\mathrm{AM} \Phi$, $242 \mathrm{IM} \Phi, \mathrm{AZU} 1+\mathrm{M} \Phi$, monocytes, $\mathrm{cDC} 1$, and $\mathrm{cDC} 2$. MregDC, meanwhile, expressed distinct 243 cytokines and chemotactic factors associated with T cell engagement, including $I L 12 B, E B I 3$, 
244 CCL17, CCL22, and CCL19, which were expressed to a minimal or greatly reduced degree in

245 monocytes, $\mathrm{M} \Phi$, or MoDC.

246

247 TCRs limited to tumors mark $\mathbf{T}$ cells with distinct phenotypic features. CITEseq

248 characterization of T cells identified populations of CD8+ cells that were characterized by an NK-

249 like signature $\left(\mathrm{T}_{\mathrm{NK}-\text { like }}\right)$, high expression of $G Z M K\left(\mathrm{~T}_{\mathrm{GZMK}}\right)$, expression of genes related to tissue-

250 residence such as ITGA1 transcript and CD103 and CD69 protein (CD8+ $\left.\mathrm{T}_{\mathrm{rm}}\right)$, and a cluster

251 consistent with activated T cells, expressing high levels of IFNG, GZMB, LAG3, CXCL13, and

252 HAVCR2 transcripts, as well as high PD-1, ICOS, and CD39 protein ( $\mathrm{T}_{\text {activated; }}$ Figure 4A, B). Other

253 clusters, which mostly consisted of CD4+ cells, could be separated into $\mathrm{T}_{\text {reg, }}, \mathrm{T}_{\mathrm{rm}}$, cells expressing

254 a profile consistent with either central memory or naïve cells ( T $_{\mathrm{CM} / \mathrm{Naiive-like-I}}$; TCF7, SELL, LEF1,

$255 M A L$, and surface expression of CD127), and a group of clusters expressing both intermediate

256 levels of this signature as well as a tissue-residency signature $\left(\mathrm{T}_{\mathrm{CM} / \mathrm{Naive}-\mathrm{like-II}}\right)$. Cells within the

$257 \mathrm{~T}_{\mathrm{CM} / \mathrm{Naive}-\text { like }}$ clusters did not otherwise segregate by signatures related to antigen experience, TCR

258 engagement, activation, or exhaustion state.

259 While clustering cells using their transcriptional profiles did not result in complete

260 separation of CD4+ and CD8+ cells, CITEseq allowed for the comparison of CD4+ versus CD8+

261 cells within otherwise transcriptionally similar groups. The $\mathrm{T}_{\text {activated }}$ cluster could therefore be

262 separated into CD4+ and CD8+ components (15.5\% and $74.8 \%$, respectively). Differential

263 expression analysis between these subsets showed that, on average, CD4+ cells in this cluster

264 expressed increased levels of $C X C L 13, C D 40 L G, B C L 6$, and IL21 (Figure S4A) consistent with a

265 phenotype similar to T-follicular-helper. We next asked whether we could use profiles of CD8+

266 and CD4+ cells within this cluster to classify CD4 and CD8 cells from samples lacking CITEseq 
surface staining, which was not available for the majority of our dataset. Learning a signature-

268 based classifier from a training set consisting of the $\mathrm{T}_{\text {activated }}$ cells from 2 patients and testing this

269 signature on the remaining patients with CITEseq staining demonstrated that transcriptional based

270 classification guided by antibody signals was highly accurate ( $86 \%$ on test set; Figure S4B). This

271 classification could further discriminate cells that uniquely expressed $C D 8-A / B$ transcripts or $C D 4$

272 transcripts across the remaining cells in the dataset (84\% accuracy; Figure S4C). Applying this

273 classification generally allowed for the separation of CD4 $\mathrm{T}_{\text {activated }}$ from CD8 $\mathrm{T}_{\text {activated }}$ across the

274 dataset (Figure S4D). Similar to a recent report ${ }^{32}$, independent quantification of these cells

275 separately and comparing their frequencies demonstrated a high correlation across tumors (Figure

$276 \mathrm{~S} 4 \mathrm{E} ; \mathrm{rho}=0.58, \mathrm{p}=2.7 \mathrm{e}-4)$, so they were continued to be grouped for further analysis.

277 While $\mathrm{T}_{\text {activated }}$ and $\mathrm{T}_{\text {reg }}$ were the most increased $\mathrm{T}$ cell populations in tumors compared to

278 nLung (Figure 4C), another cluster, characterized by high expression of cell-cycle genes MKI67

279 and STMN1, and surface expression of HLA-DR and CD38, was also significantly increased in

280 tumors $\left(\mathrm{T}_{\text {cycle }}\right.$; Figure $\left.4 \mathrm{~A}-\mathrm{C}\right)$. Other than expressing these hallmarks of proliferation, the $\mathrm{T}_{\text {cycle }}$

281 cluster was diverse with respect to RNA and protein expression (Figure 4A, B). Analyzing the

282 cells comprising $\mathrm{T}_{\text {cycle }}$ by gene scores constructed from genes differentially expressed among the

283 other clusters demonstrated that $\mathrm{T}_{\text {cycle }}$ is a mixture of multiple $\mathrm{T}$ cell phenotypes that share the

284 cycling state (Figure S4F and Table S4). While tumors expressed overall higher frequencies of 285 cycling $\mathrm{T}$ cells (Figures $4 \mathrm{C}$ and $\mathrm{S} 4 \mathrm{G}$ ), $\mathrm{T}_{\text {activated }}$ and $\mathrm{T}_{\text {reg }}$ showed the highest frequencies of cycling 286 cells compared to other phenotypes (Figure S4H).

287 To understand the clonal relationships among $\mathrm{T}$ cell phenotypes in tumor and nLung

288 tissues, we performed paired scRNAseq and TCRseq using a nested PCR approach on paired 289 tissues from 3 patients. Classification of the transcriptomes among the clusters and analysis of the 
290 T cell repertoires among these phenotypes confirmed that cells mapping to the $T_{\text {activated }}$ cluster were

291 the most clonal population in tumors (Figure S4I). Furthermore, dividing clones into groups based

292 on their expansion in nLung or tumor determined the phenotypes of shared clones compared to

293 clones detected in either tissue specifically (Figures 4D, E,

294 S4J, K). In nLung samples, the phenotypic distribution of T cells with TCRs either shared with

295 tumor samples or only present only in nLung was similar (Figure 4Ei). In tumors, however, we

296 observed differences in phenotypic distributions between cells with shared versus tissue-specific

297 TCRs (Figure 4Eii). Specifically, no $\mathrm{T}_{\mathrm{NK}-\text { like }}$ cells in tumors had TCRs that were uniquely expanded

298 in tumors. Furthermore, the proportion of $\mathrm{T}_{\text {cycle, }} \mathrm{T}_{\text {reg, }}$, and $\mathrm{T}_{\text {activated }}$ among cells with TCRs uniquely

299 expanded in tumors were all markedly increased compared to their proportions among cells with

300 TCRs present in both tissues, and these relationships were not observed in nLung. By controlling

301 for the distribution of cells with shared TCRs in the tumor, we found that the clonal enrichment in

302 these populations was not simply due to enrichment of these phenotypes within tumor. Together,

303 the finding that $\mathrm{T}_{\text {activated }}$ are enriched in clonally expanded and cycling $\mathrm{T}$ cells at the tumor suggests

304 that their accumulation is at least in part due to local clonal expansion.

305

$B$ cells and plasma cells are increased in tumors, but the B:plasma cell ratio is conserved

307 between tumor and nLung.

308 B and plasma cells represented the most globally increased lineage among immune cells in tumors

309 compared to nLung across multiple datasets (Figure 1C-E); B cells were increased as a proportion

310 of immune cells by a median of 6.4-fold (IQR: 2.5-8.4), while plasma cells were similarly

311 increased by a median of 4.1-fold (IQR: 2.2-9.4). B cells and plasma cells were strongly distinct

312 both on the RNA and surface marker level (Figure 4F, G). Plasma cell clusters included rare IgD+ 
313 plasma cells, which were also the only CD38 ${ }^{\text {int }}$ population. B cell frequencies overwhelmed plasma

314 cell frequencies with $\mathrm{IgD}+$ and $\mathrm{IgM}+$ plasma cells being the rarest, but lineage-normalized

315 frequencies were not different between nLung and Tumor (Figures 1E and S4L). B cells and

316 plasma cells were therefore found to increase in tumors without significant overall perturbation of

317 the B:plasma cell ratio or plasma cell isotype ratios.

Ligand-receptor interactions identify potential drivers of an adaptive activation module. In

320 order to identify links between cellular phenotypes that may drive patient diversity, we performed

321 correlation analyses across cell type frequencies in tumors normalized within lineage (Figure 5A).

322 Among the most highly correlated cell types were $\mathrm{T}_{\text {activated, }} \mathrm{IgG}+$ plasma cells, and MoMФ-II; we

323 therefore called these cell types collectively the lung cancer activation module (LCAM). The cell

324 types that were most anticorrelated to this module included $\mathrm{B}$ cells, $\mathrm{T}_{\mathrm{cm} / n \text { niive-II }}, \mathrm{AM} \Phi$, resting cDC,

325 and AZU1+ MФ. Sorting patients by these cell types revealed that patients could be broadly

326 grouped into those with high or low frequencies of LCAM cell types (Figure 5B). We called these

327 groups $\mathrm{LCAM}^{\text {hi }}$ and $\mathrm{LCAM}^{\text {lo }}$, respectively. Including samples from external datasets in this

328 stratification supported the overall pattern (Figure 5B and S5A). This stratification was not

329 strongly associated with changes in lineage frequencies among total immune cells, and

330 accordingly, samples from both $\mathrm{LCAM}^{\mathrm{hi}}$ and $\mathrm{LCAM}^{\mathrm{lo}}$ groups generally displayed lineage-

331 population shifts in line with overall tumor versus nLung differences, such as decreased NK and

332 increased B lineage frequencies (Figure 5C). Therefore, while LCAM cell types included some of

333 the populations that were most enriched in tumor compared to nLung on average, the LCAM axis

334 was importantly not a reflection of tumor sample purity. 
To identify tumor-specific immune dysregulation that may contribute to shaping the

336

337

338

339

340

341

342

343

344

345

346

347

348

349

350

351

352

353

354

355

356

357

LCAM $^{\text {hi }}$ vs. LCAM ${ }^{\text {lo }}$ cellular organization among patients, we performed an unbiased analysis of ligand-receptor pairs between immune subsets, leveraging a dataset of secreted ligands and their experimentally validated receptors ${ }^{16,33}$ (Figure S5B, C and Table S6), comparing differences in ligand-receptor (LR) intensity scores ${ }^{16}$ between $\mathrm{LCAM}^{\mathrm{hi}}$ and $\mathrm{LCAM}^{\mathrm{lo}}$ groups, as well as between each group and their respective adjacent nLung tissues (Table S7). Overall, both LCAM ${ }^{\text {hi }}$ and $\mathrm{LCAM}^{\mathrm{lo}}$ patients demonstrated correlated modes of LR activation in tumors compared to nLung (Figure 5D). In particular, tumors in both LCAM $^{\text {hi }}$ patients and LCAM $^{\text {lo }}$ patients exhibited strong intensity scores between T-cell derived CXCL13 and B cell CXCR5, which is likely contributing to the influx of B and plasma cells seen in tumors (Figure 5E). T cells in LCAM ${ }^{\text {hi }}$ but not LCAM ${ }^{\text {lo }}$ patients also produced other factors in tumors but not nLung capable of stimulating B cells through the IFNG-IFNGR1 axis and the BTLA-TNFRSF14 axis (Figure 5E). In addition, B cells from LCAM $^{\text {hi }}$ but not LCAM ${ }^{\text {lo }}$ patients highly expressed TNFSF9 (41BBL), which ligates TNFRSF9 (41BB), that we found highly expressed on $\mathrm{T}_{\text {activated }}$ cells (Figure S5D), indicating B cells from LCAM $^{\text {hi }}$ patients participate in activation of T cells via TNFSF9-TNFRSF9 interaction.

In addition, we observed increased IFNG-IFNGR signaling between T cells and myeloid cells in LCAM $^{\text {hi }}$ patients (Figures 5F and S5E, F). Potentially in result, LCAM ${ }^{\text {hi }}$ patients displayed higher activation of the CXCL9/10/11-CXCR3 axis between myeloid and T cells (Figure 5G-I). Whereas MФ and MoDC demonstrated many conserved ligands upregulated in both LCAM $^{\text {hi }}$ and $\mathrm{LCAM}^{\mathrm{lo}}$ tumors such as IL10 and OSM (Figure 5G, H; note distribution of highlighted LR pairs along the diagonal), tumor $\mathrm{cDC}$ shared few ligands between the two groups, and rather upregulated CCL19 higher in LCAM ${ }^{\text {hi }}$ patients compared to CCL17 in LCAM ${ }^{\text {lo }}$ patients (Figure 5I); MoDC also demonstrated the latter pattern, selectively expressing CCL17 in LCAM ${ }^{\text {lo }}$ patients (Figure 
$5 \mathrm{H})$, suggesting that DC expression of CCL19 may be a unique feature of cDC necessary for activation of $\mathrm{T}$ cells as well as induction of a humoral immune response. Overall, differences in ligand-receptor intensity scores between $\mathrm{LCAM}^{\mathrm{hi}}$ and $\mathrm{LCAM}^{\mathrm{lo}}$ patients supported such a patient stratification, and provide possible mechanistic insight into immune-cell crosstalk underlying the development of the LCAM axis, including IFNg signaling as a major driver.

Projection of bulk-transcriptomic data onto scRNA-derived signatures reveals the presence

366 To identify tumor-related correlates of the LCAM module, we aimed to analyze a larger patient cohort in order to increase statistical power. Therefore, we implemented an unbiased method of scoring bulk transcriptomic signatures along the LCAM axis ${ }^{16,34}$. Specifically, we identified genes that were both differentially expressed between LCAM $^{\text {hi }}$ and LCAM $^{\text {lo }}$ tumor samples (Figure S6A), and also highly specific to the cell types enriched or depleted in the LCAM ${ }^{\text {hi }}$ tumors (Figure 371 S6B, see methods). Using a published tabulation on estimated immune content of 512 lung 372 adenocarcinoma (LUAD) patients available from TCGA based on expression of immune genes of

373 all lineages ${ }^{35}$, we saw as expected that scores generated with either gene set were highly correlated

374 with estimates of overall immune content (Figure S6C, D), but an ensemble LCAM score 375 computed by the difference of these scores (LCAM ${ }^{\text {hi }}$ score - LCAM ${ }^{\text {lo }}$ score) was not (Figure S6E).

376 As predicted by our scRNAseq data, when controlling for the immune content, we generally 377 observed negative correlations of $\mathrm{LCAM}^{\mathrm{hi}}$ and $\mathrm{LCAM}^{\mathrm{lo}}$ gene scores among tumors except for 378 samples with the $10 \%$ lowest immune content (Figure S6F, G), suggesting that the ensemble 379 LCAM score might measure a mode of immune activation that is independent of the overall 380 immune infiltration measured by immune content. We excluded the samples with the lowest $10 \%$ 
381 immune content from further analysis because probing the immune signatures was likely less

382 informative within these samples. Sorting the patients by the ensemble LCAM score revealed the 383 presence of $\mathrm{LCAM}^{\mathrm{hi}}$ and $\mathrm{LCAM}^{\mathrm{lo}}$ patient groups within the cohort (Figure 6A).

384 To see whether a similar pattern was present in additional datasets, we probed the independent CPTAC: LUAD cohort, consisting of 110 treatment-naive LUAD patients undergoing surgical resection on whom bulk RNAseq and WES had been performed (Michael A. Gillette, et revealed the presence of $\mathrm{LCAM}^{\mathrm{hi}}$ and $\mathrm{LCAM}^{\mathrm{lo}}$ patient groups (Figure S6H), further establishing the prevalence of this cellular module in a subset of LUAD patients.

\section{LCAM immune response correlates with tumor-genotype and expression of tumor-antigens}

\section{in LUAD lesions}

393 While the anti-tumor immune response can be modulated by many tumor-intrinsic and tumor-

extrinsic factors, the tumor-infiltrating immune cells exist as part of a complex microenvironment

that includes many other stromal populations ${ }^{13,36}$. To ask whether the ensemble LCAM score is

396 associated with other non-tumor, non-immune stromal populations, we derived gene lists that were

397 specific for individual stromal populations identified in a public dataset of 8 NSCLC patients ${ }^{5}$

398 (Table S4), and used these genes to quantify enrichment of stromal populations in TCGA LUAD

399 data. The ensemble LCAM score correlated with a cancer-associated fibroblast (CAF) enrichment

400 score, anticorrelated with a normal fibroblast enrichment score, and strongly correlated with the

401 difference of these scores (Figure 6B-D). Meanwhile, it exhibited weak or absent correlations with

402 a tumor-associated blood endothelial cell (BEC) enrichment score, an nLung BEC enrichment

403 score, and a lymphatic endothelial score (Figure S6I). These data suggest an intimate link between 
404 development of the LCAM cellular module and a CAF-like fibroblast phenotype, which should be

405 explored in further detail as CAF have been suggested to act as major regulators of TIL 406 function $^{36,37}$.

407 We hypothesized that variability in immune and stromal states captured by the LCAM and 408 CAF signatures could be associated with different tumor properties. While the ensemble LCAM 409 score demonstrated a small but significant increase in large tumors $(t=2.60, p=0.01$ between TNM $410 \mathrm{~T}$-stage $=\mathrm{T} 1$ and $\mathrm{T}$-stage $>\mathrm{T} 1$ ), we observed variable LCAM presence among tumors of all stages

411 (Figure S6J). Furthermore, while PD-L1 expression is the most commonly used biomarker guiding 412 ICB treatment, we also observed a weak correlation between the ensemble LCAM score and total 413 CD274 expression $(\mathrm{r}=0.21, \mathrm{p}=2.7 \mathrm{e}-5)$. TMB, meanwhile, has been demonstrated to be one of the 414 most robust predictors of checkpoint response ${ }^{38}$, and is supported by the key mechanistic 415 hypothesis that tumors with many mutations more easily activate and are targeted by the immune 416 system via the generation of mutated peptides and damage-associated molecular patterns. 417 Strikingly, the data showed that the ensemble LCAM score was strongly correlated with TMB 418 both in TCGA (Figure 6E; $r=0.47 \mathrm{p}<2.2 \mathrm{e}-16)$ and in CPTAC (Figure $\mathrm{S} 6 \mathrm{~K})(\mathrm{r}=0.53 \mathrm{p}=2.3 \mathrm{e}-9)$. By 419 comparison, other scores measuring overall immune content (Immune ESTIMATE ${ }^{35}$ ) or specific 420 aspects of immune state (T cell-inflamed gene expression profile (GEP) score ${ }^{39,40}$ ) had much 421 weaker associations with TMB (Figure S6L, M). Importantly, correlation with TMB was observed 422 broadly across LCAM $^{\mathrm{hi}}$ genes expressed in multiple cell types, whereas conversely, anti423 correlation with TMB was also observed broadly across LCAM ${ }^{\text {lo }}$ genes (Figure S6N). The 424 ensemble LCAM score correlated with TMB to similar extents among patients grouped within 425 each TNM T-stage (Figure S6O). 
In LUAD cases, TMB is strongly associated with smoking history. Consistent with this

427 relationship, the ensemble LCAM score correlated with smoking pack-years $(\mathrm{rho}=0.23, \mathrm{p}=4.4 \mathrm{e}-5)$.

428 Therefore, smoking history confounded the correlation we observed between TMB and the

429 ensemble LCAM score, suggesting that the immune signature could be only indirectly related to

430 mutations and specifically mutated neoantigens, but rather due to alternate modes of immune

431 dysregulation related to smoking exposure. To test this hypothesis, we stratified tumors by the

432 detection of the smoking-related mutational signature characterized by $\mathrm{C}>\mathrm{A}$ de-aminations within

433 specific trinucleotide contexts ${ }^{41,42}$. This approach removed uncertainty related to unreliability of

434 patient-reported smoking statistics and missing clinical data. We observed that both tumors with

435 and without detection of this signature exhibited significant correlations between TMB and the

436 ensemble LCAM score $(r=0.38 ; p=9.2 e-5$ in the undetected smoking signature group) despite

437 having clearly distinct distributions of TMB (Figure 6E), suggesting that this relationship was

438 independent of smoking-driven immunomodulation.

We then asked which additional features of the tumors may influence the ensemble LCAM

440 score beyond the effect caused by differences in TMB. To perform this analysis, we regressed the

441 ensemble LCAM score onto the LogTMB and correlated candidate variables with regression

442 residuals, which quantify the difference between the observed and expected LCAM scores based

443 on this relationship. For example, scores quantifying total predicted single-nucleotide-variant- or

444 Insertion/deletion-induced neoantigens did not correlate with these differences (Figure S6P, Q),

445 indicating that these neoantigen prediction scores did not provide more information regarding the

446 LCAM immune modulation than TMB alone. However, consistent with the hypothesis that

447 generation of tumor-associated antigens was the key mechanism connecting TMB to an LCAM

448 response, we found that a score quantifying total tumor associated but not tumor-specific cancer- 
449 testis antigens (CTA) was correlated with the regression residuals (Figure 6F; r=0.16, $\mathrm{p}=3.4 \mathrm{e}-3$ ),

450 suggesting that additional tumor-associated antigens beyond those directly caused by tumor

451 mutations may also contribute to induction of the LCAM response.

452 Most adenocarcinoma patients have at least one of a small number of common driver

453 mutations, including KRAS, EGFR, STK11, and TP53. Recently, it was shown that LUAD patients

454 responsive to immune checkpoint blockade frequently have tumors harboring TP53 mutations, and

455 that TP53 mutant status was associated with enrichment of CD8 T cells in the TME ${ }^{43,44}$. However,

456 immune-related effects of individual mutations have generally not been considered independently

457 given their correlation with TMB. Specifically, while TP53-mutant tumors had higher ensemble

458 LCAM scores compared to TP53-WT/(EGFR or KRAS or STK11)-mut tumors in both TCGA and

459 CPTAC datasets (Figures 6G and S6R), TP53 was also most strongly associated with increased

460 TMB (Figures 6H and S6S). In order to statistically test whether these mutations were associated

461 with higher LCAM scores while controlling for TMB, we regressed the LCAM score onto the

462 LogTMB and asked whether any individual mutations were correlated with the regression

463 residuals. Interestingly, this analysis showed that TP53-mutant patients had higher LCAM scores

464 than expected by a model assuming only correlation with TMB (Figure 6I; p=1.4e-3). KRAS-

465 mutant patients, meanwhile, had lower LCAM scores than expected by this model (Figure 6J;

$466 \mathrm{p}=1.6 \mathrm{e}-4)$. There was no similar deviation seen in either STK11- or EGFR-mutant patients (Figure

467 S6T). Overall, projection of bulk signatures onto axes defined by variation in our scRNAseq cohort

468 suggested that expression of the LCAM cellular module is a marker of adaptive response against

469 mutated and ectopically-expressed tumor-associated antigens that is independent from the overall

470 level of immune infiltration. 


\section{DISCUSSION}

473 The analysis of matched tumor and nLung tissues from 35 patients as described here provides the

474 largest unbiased single-cell map of the immune response of early-stage lung cancer lesions to date.

475 CITEseq analysis, combining phenotypic classifications based on surface protein expression with

476 transcriptomic profile, serves here to help unite high-dimensional models of cellular classification

477 and refine our understanding of the immune cellular landscape in disease lesions. By further

478 integrating tumor and nLung samples from public datasets, we demonstrated the robustness of the

479 reported signatures across platforms. Importantly, based on high levels of changes conserved

480 across tumor lesions, these data support the notion that common immunotherapy treatment

481 paradigms could be beneficial for large subsets of patients despite existing disease heterogeneity.

482 Among tumors, patients could, however, be stratified along a dominant LCAM axis that

483 was independent of overall immune infiltration or changes in proportions of immune lineages.

484 This axis was defined by a high level of $\mathrm{IgG}+$ plasma cells, activated $\mathrm{T}$ cells that were clonally

485 enriched in the tumor and expressing a proliferation signature, and MoMac-II that expressed SPP1,

486 a glycolysis signature, and a set of inflammatory secreted factors; this module of cell types

487 anticorrelated with B cells, T cells with a Tcm/naïve-like phenotype, resting cDC, AMФ, and MФ

488 expressing $A Z U 1$. We therefore propose that LCAM $^{\text {hi }}$ patients are undergoing a more vigorous

489 antigen-specific antitumor adaptive immune response, whereas LCAM $^{\text {lo }}$ patients fail to mount an

490 adaptive response to such a degree. Unbiased ligand-receptor analyses showed that, while both

491 LCAM $^{\text {hi }}$ and LCAM ${ }^{\text {lo }}$ tumors expressed similar patterns of ligand-receptor pairs among immune

492 cells compared to nLung, LCAM $^{\text {hi }}$ status was specifically related to heightened CXCL13

493 expression by $\mathrm{T}$ cells, IFNg signaling from $\mathrm{T}$ cells to myeloid and B cells, and CXCL-9,10,11

494 signaling from myeloid cells; cDC meanwhile expressed more CCL19 in LCAM ${ }^{\text {hi }}$ tumors 
compared to more CCL17 in LCAM ${ }^{\mathrm{lo}}$ tumors. These factors likely served to modify the immune

496 response around a set of conserved changes compared to nLung observed in both LCAM $^{\text {hi }}$ and

$497 \quad$ LCAM $^{\text {lo }}$ tumors.

498 When analyzed in the context of broader datasets with paired bulk transcriptomics and

499 whole exome sequencing, an ensemble gene score learned from the $\mathrm{LCAM}^{\mathrm{hi}}$ and $\mathrm{LCAM}^{\mathrm{lo}}$ patients

500 and associated cell types strongly correlated with measures indicative of high levels of tumor-

501 associated antigens, namely TMB and a cancer testis antigen score. Interestingly, the LCAM score

502 was not correlated with the overall immune infiltrate, and was independent of the T-cell inflamed

503 gene expression profile score commonly used to reflect immune activation in tumor lesions ${ }^{39,40}$.

504 While the ensemble LCAM score was correlated with smoking status and weakly correlated with

505 stage, the relationship with TMB remained even after controlling for these possible confounders.

506 Given that TMB has demonstrated predictive power with response to ICB response in NSCLC ${ }^{14,38}$,

507 the relationship between TMB and the LCAM cellular module in treatment-naïve patients suggests

508 that this effect may be mediated via a conditioning of the immune system that exists prior to

509 treatment, and that measurement of this cellular module may provide a more direct indicator with

510 respect to the immune system's propensity for ICB response. Specifically, the fact that many

511 factors significantly influence the LCAM score, not just TMB, demonstrates how the immune

512 system integrates multiple types of signals to establish its set point.

513 Importantly, while previously reported immune signatures have been proposed to reflect

514 tumor cytolytic activity or $\mathrm{T}$ cell and IFNg-driven immune response in association with tumor

515 antigens and immune evasion modes ${ }^{39,40,45}$, the LCAM axis presented here represents an integrated

516 assessment of the immune cellular organization, based on all immune cell types as defined by

517 scRNA across patients, likely arising as a direct response to tumor antigens. 
An additional question of clinical interest relates to how different driver mutations affect

519 the conditioning of the immune system and ICB response. The analysis presented here shows that

520 the common LUAD driver mutations EGFR and STK11 had little effect on the LCAM response

521 beyond that explained by their association with TMB. While STK11 mutation status has been

522 shown to be the most prevalent genomic driver of primary resistance to $\mathrm{ICB}^{44,46}$, there were no

523 patients with STK11-mutated tumors in our scRNAseq cohort, so this effect can therefore not be

524 addressed here. Meanwhile, compared to what was expected based on each tumor's TMB alone,

525 TP53 mutation intensified the LCAM response and KRAS mutation blunted it. Interestingly, the

526 latter result is consistent with a recent report demonstrating that pharmacological blockade of

527 KRAS-G12C in preclinical studies resulted in a robust immune response and synergized with

528 anti-PD1 treatment ${ }^{47}$. The mechanisms of these effects remain to be seen, and may relate to the

529 expression of immunomodulatory factors by the tumor, or the re-shaping of the metabolic

530 microenvironment, for example. To elucidate such pathways, close study of the tumor on a broader

531 molecular scale, in conjunction with the immune cell composition and state, is necessary.

A further, surprising result from our bulk RNA analyses was that the LCAM axis was

533 highly consistent with a change in fibroblast phenotype based on signatures derived from

534 scRNAseq of NSCLC stromal clusters ${ }^{5}$. This association could suggest that the development of

535 the tumor fibroblast phenotype is in response to overwhelming immune activation that may be 536 instigated by an adaptive, antigen-specific response.

537 An important limitation of these findings relates to the site of initiation of the LCAM

538 response; while the LCAM cellular module consists of cells undergoing an apparent active immune

539 response, this study does not demonstrate the extent to which the module is instigated or

540 perpetuated in situ at the tumor lesion. Specifically, despite evidence of clonally expanding $\mathrm{T}_{\text {activated }}$ 
541 cells, it remains unclear whether these lineages are primed in situ versus in tumor-draining lymph

542 nodes (TdLN). While understanding the timescale of the tumor specific response will always be

543 challenging due to variation in patient presentation timelines, it will nevertheless be important to

544 correlate the cell types and states present in the TdLN in order to determine whether the LCAM

545 response depends on lymph node priming, as well as to develop a deeper understanding of the

546 spatial dynamics of the LCAM cell types.

547 Overall, the model presented here identifies an immune activation signature, derived from

548 definitions of immune phenotypes defined by single-cell RNA and CITEseq, as an integrator of

549 tumor-associated antigen load and driver mutation status that is not related to overall immune

550 content. We believe that this axis, therefore, can serve as a more direct measure of antigen-specific,

551 anti-tumor immune activation compared to previously suggested immune readouts. 


\section{METHODS}

\section{Human subjects}

556 Samples of tumor and non-involved lung were obtained from surgical specimens of patients 557 undergoing resection at Mount Sinai Hospital (New York, NY) after obtaining informed consent

558 in accordance with a protocol reviewed and approved by the Institutional Review Board at the 559 Icahn School of Medicine at Mount Sinai (IRB Human Subjects Electronic Research Applications 560 10-00472 and 10-00135) and in collaboration with the Biorepository and Department of Pathology.

\section{Tissue processing}

563 Tissues were rinsed in PBS, minced and incubated for 40 minutes at $37^{\circ} \mathrm{C}$ in Collagenase IV

$5640.25 \mathrm{mg} / \mathrm{ml}$, Collagenase D 200U/ml and DNAse I 0.1mg.ml (all Sigma). Cell suspensions were 565 then aspirated through a $18 \mathrm{G}$ needle ten times and strained through a 70 -micron mesh prior to RBC 566 lysis. Cell suspensions were enriched for $\mathrm{CD}^{4} 5^{+}$cells by either bead positive selection (Miltenyi) 567 per kit instructions or FACS sorting on a BD FACSAria flow sorter (as indicated in Table S1) 568 prior to processing for scRNAseq or CITEseq.

\section{ScRNA- and TCR-seq}

571 For each sample, 10,000 cells were loaded onto a 10X Chromium single-cell encapsulation chip 572 according to manufacturer instructions. Kit versions for each sample are indicated in Table S1.

573 Libraries were prepared according to manufacturer instructions. QC of cDNA and final libraries 574 was performed using CyberGreen qPCR library quantification assay. Sequencing was performed 575 on Illumina sequencers to a depth of at least 80 million reads per library. 
TCRseq was performed using the Chromium Single Cell 5' VDJ kit, following

577 manufacturer's instructions. For patients 695 and 706, cells were subject to a CD2+ bead

578 enrichment (Miltenyi) instead of CD45+ enrichment prior to encapsulation.

\section{CITEseq}

581 For each sample, cell suspensions were split and barcoded using "hashing antibodies"48 staining 582 beta-2-microglobulin and CD298 and conjugated to "hash-tag" oligonucleotides (HTOs). Hashed 583 samples were pooled and stained with CITEseq antibodies that had been purchased either from the

584 Biolegend TOTALseq catalog or conjugated using the Thunder-Link PLUS Oligo Conjugation kit

585 (Expedeon). Sample hashing schemes and CITEseq panels are detailed in Tables S1 and S2, 586 respectively. Stained cells were then encapsulated for single-cell reverse transcription using the 587 10X Chromium platform and libraries were prepared as previously described ${ }^{15}$ with minor 588 modifications. Briefly, cDNA amplification was performed in the presence of $2 \mathrm{pM}$ of an antibody589 oligo specific primer to increase yield of antibody derived tags (ADTs). The amplified cDNA was 590 then separated by SPRI size selection into cDNA fractions containing mRNA derived cDNA 591 (>300bp) and ADT-derived cDNAs (<180bp), which were further purified by additional rounds of 592 SPRI selection. Independent sequencing libraries were generated from the mRNA and ADT cDNA 593 fractions, which were quantified, pooled and sequenced together on an Illumina Nextseq to a depth 594 of at least 80 million reads per gene expression library and 20 million reads per ADT library.

596 MICSSS

597 FFPE tissues were stained using multiplexed immunohistochemical consecutive staining on a 598 single slide as previously described ${ }^{22}$. Briefly, slides were baked at $37^{\circ} \mathrm{C}$ overnight, deparaffinized 
599 in xylene, and rehydrated in decreasing concentrations of ethanol. Tissue sections were incubated

600 in citrate buffer ( $\mathrm{pH} 6$ or 9) for antigen retrieval at $95^{\circ} \mathrm{C}$ for 30 minutes, followed by incubation in

$6013 \%$ hydrogen peroxide and in serum-free protein block solution (Dako, X0909) before adding

602 primary antibody for 1 hour at room temperature. After signal amplification using secondary

603 antibody conjugated to streptavidin-horseradish peroxidase and chromogenic revelation using 3-

604 amino-9-ethylcarbazole (AEC), slides were counterstained with hematoxylin, mounted with a

605 glycerol-based mounting medium and scanned for digital imaging (Pannoramic 250 Flash III

606 whole-slide scanner, 3DHISTECH). Then the same slides were successively bleached and re-

607 stained as previously described ${ }^{22}$. Primary antibodies were: anti-human CD10 (200103, R\&D

608 systems), DC-Lamp (1010E1.01, Novus biologicals), pan-cytokeratin (AE1/AE3, Dako), PDPN

609 (D@-40, Ventana), CD163 (10D6, Novus Biologicals) and PD-L1 (E1L3N, Cell Signaling Tech).

\section{Analysis of Sequencing data}

612 Transcriptomic and TCR library reads were aligned to the GRCh38/84 reference genome and

613 quantified using Cellranger (v3.1.0). CITEseq ADT and CITEseq HTO reads were queried for

614 antibody- and cell-specific oligonucleotide sequence barcodes in the designated read positions,

615 including antibody sequences within a Hamming distance of 1 from the reference, using the

616 feature-indexing function of Cellranger. Resulting alignment statistics are reported in Table S3.

617 TCR data was aligned using Cellranger $v d j$ function with default parameters.

\section{CITEseq processing and normalization}

620 Doublets were removed based on co-staining of distinct sample-barcoding ("hashing") antibodies

621 ([maximum HTO counts $] /\left[2^{\text {nd }}\right.$ most HTO counts $\left.]<5\right)$ and cell barcodes with few HTO counts 
622 (maximum HTO counts < 10) were also excluded. Cells were then assigned to samples based on

623 their maximum staining HTO. HTO to sample associations are detailed in Table S1.

624 To normalize ADT counts across experimental batches given different CITEseq staining

625 panels and sequencing runs, we performed a quantile-normalization on the ADT count values for

626 each surface marker for the immune cells in each $10 \mathrm{X}$ encapsulation batch. To do this, the

627 geometric average of the quantile function was computed across batches

628

$$
\overline{F_{m}^{-1}(p)}=\left(\prod_{b=1}^{N}\left[F_{m, b}^{-1}(p)+d\right]\right)^{\frac{1}{N}}
$$

629 where $F_{m, b}^{-1}(p)$ is the quantile function, or inverse cumulative distribution function, for counts of

630 CITEseq marker $m$ on immune cells in each of $N 10 \mathrm{X}$ encapsulation batches $b$ and regularization

631 factor $d=1$ ADT count, evaluated at quantile $p$ in interval $[0,1]$. This geometric average quantile

632 function provided a reference function for a common mapping of cells based on their single-

633 channel, batch-specific staining quantile $p$ to a normalized staining intensity. Of note, this

634 normalization method preserved the relationships between channels while constraining the

635 observed differences in staining across experiments within individual channels.

\section{Unsupervised batch-aware clustering analysis}

638 Immune cells from tumor and nLung samples were filtered for cell barcodes recording > 500 UMI,

639 with $<25 \%$ mitochondrial gene expression, and with less than defined thresholds of expression

640 for genes associated with red blood cells and with epithelial cells (Table S4). Cells were clustered

641 using an unsupervised batch-aware clustering method we have recently described ${ }^{16}$ with minor

642 adjustments. This EM-like algorithm, which was also based on earlier studies ${ }^{49,50}$, iteratively

643 updates both cluster assignments and sample-wise noise estimates until it converges, using a 
644 multinomial mixture model capturing the transcriptional profiles of the different cell-states and

645 sample specific fractions of background noise. We clustered $19 \mathrm{nLung}$ and 22 tumor samples

646 jointly and 46 additional tumor and nLung samples were mapped onto the final model as described

647 below.

648 The model definitions and estimation of model parameters were as described in $\left({ }^{16}\right)$.

649 Specifically, the probability of observing gene $i$ in cell $j$ is defined as:

650

$$
p_{j i}=\frac{1}{Z}\left[K_{r e g}+\left(1-\eta_{b^{j}}\right) \cdot \alpha_{i, m a p^{j}}+\eta_{b^{j}} \cdot \beta_{i, b^{j}}\right]
$$

651 Where $\operatorname{map}^{j}$ and $b^{j}$ are assignments of cells $j$ to cell-type and batch respectively; $\eta_{b^{j}}$ is the fraction

652 of UMIs contributed by background noise; $\alpha_{i, m a p}$ is the probability that a molecule drawn from

653 celltype $m a p^{j}$ is of gene $\mathrm{i}$ (assuming no background noise) $\beta_{i, b^{j}}$ is the probability that a noise UMI

654 drawn from batch $b^{j}$ will be of gene $i$, and $K_{\text {reg }}$ is a small regularization constant.

655 We also used here the pseudo expectation-maximization (EM) algorithm ${ }^{16}$ to infer the 656 model parameters with minor modifications: (1) training set size was 2000 instead of 1000 cells 657 and (2) the best clustering initiation was selected from 1000 instead of 10000 kmeans + runs. For 658 this clustering we included barcodes with more than 800 UMIs and used $K_{\text {reg_ds }}=0.2 ;\left(\mathrm{P}_{1}, \mathrm{P}_{2}\right)=$ $659\left(0^{\text {th }}, 30^{\text {th }}\right)$ percentiles; $K_{\text {reg }}=5 \cdot 10^{-6} ; \mathrm{k}=60$. Genes with high variability between patients were 660 not used in the clustering. Those genes consisted of mitochondrial, stress, metallothionein genes, 661 immunoglobulin variable chain genes, HLA class I and II genes and 3 specific genes with 662 variable/noisy expression: MALAT1, JCHAIN and XIST (Table S4). Ribosomal genes were 663 excluded only from the k-means clustering (Step 2.D as described in $\left({ }^{16}\right)$ ). Samples used to 664 generate this model included only those that were enriched for CD45+ immune cells using bead 665 enrichment and were processed with the 10X Chromium V2 workflow. 


\section{Integration of additional single-cell data}

668 The resulting clustering model was used to analyze additional data that was both generated in-

669 house or downloaded from public datasets. Single cells were mapped to clusters defined by the

670 previously generated model $\alpha$. Similarly to the clustering iterations, this process associates single-

671 cells of a sample with multinomial probability vectors defined by the model and estimates the

672 noise fractions of the sample $\eta_{b}$ by optimizing the likelihood function $\left({ }^{16}\right)$ :

673

$$
f\left(\eta_{b}\right)=\sum_{j} \sum_{i} U_{i j} \log \left(p_{j i}\right)
$$

674 For $p_{j i}$ as defined above, while $\alpha_{i, m a p^{j}}$ are updated using maximum likelihood.

675 Integrating inDrop data from $\left(^{6}\right)$ and $10 \mathrm{X}$ Chromium 5' data required addressing the

676 systematic differences ${ }^{51}$ in gene capture present between these technologies and 10X Chromium

677 3' data that was used to develop the clustering model. Analysis of the differences in gene 678 expression between the technologies suggested that a multiplicative correction factor $C_{i}$ per each

679 gene $i$ could adjust for the capture efficiency differences. The following process was used to 680 estimate the correction parameters:

681 1. Map cells to the original cluster models, as above, assuming absent noise in order to prevent 682 the estimated noise term from being driven by error due to batch differences instead of true 683 noise.

684 2. Re-calculate models using the average expression of the mapped cells for each cluster to 685 form "data-based models" $\alpha^{D}$.

686 3. Calculate a weight matrix $W$, that weights individual genes for each cluster. $W$ is calculated 687 by

$$
W_{i, j}=\max \left(\alpha_{i, j}, \alpha_{i, j}^{D}\right)+w_{r e g}
$$


for original cluster model matrix $\alpha$, data-based cluster model $\alpha^{D}$, gene $i$, cluster $j$, and regularization constant $w_{\text {reg }}=10^{-10}$. Since highly detected genes tend to dominate the mapping results, it is important to account for genes that are highly detected in either the original (10X Chromium V2) platform or the new platform

4. Construct a vector of gene-specific conversion factors that can operate between platforms:

$$
C_{i}=\sum_{j} W_{i, j}\left(\left[c+\alpha^{D}{ }_{i, j}\right] /\left[c+\alpha_{i, j}\right]\right)
$$
for regularization factor $\mathrm{c}=10^{-6}$.

5. Generate transformed cluster models $\alpha^{\prime}{ }_{i, j}$ by multiplying the original models by the conversion vector and dividing by a normalization factor $\mathrm{Z}$ :

$$
\alpha_{i, j}^{\prime}=\frac{1}{Z} * C_{i} \alpha_{i}{ }_{i}
$$

6. Map cells to transformed models without fixing the noise. correspondence between the model and the mapped samples across the different technologies.

703 (Fig S1B).

704

\section{Analysis of public datasets}

706 Fastqs of scRNAseq data of tumors and nLung from 8 NSCLC patients ${ }^{5}$ acquired using 10X

707 Chromium protocols was downloaded from ArrayExpress accessions E-MTAB-6149 and E-

708 MTAB-6653. Sequencing reads were re-aligned using Cellranger as described above . Single-cells

709 were mapped to clusters as described above. Tumor samples included 3 separate samples from the

710 core, middle, and edge of each tumor. Regional tumor samples were considered separately for the 
711 intra- versus inter-patient variability analyses (Figure S1G, H). For remaining analyses, cell counts

712 of projected tumor samples were pooled by patient.

713 scRNAseq data of tumors from 7 NSCLC patients ${ }^{6}$ acquired using inDrop was downloaded

714 from GEO accession GSE127465. Since neutrophils were not detected in 10X Chromium data,

715 cells that were annotated as neutrophils in the

716 GSE127465_human_cell_metadata_54773x25.tsv.gz file were excluded from analysis. Cells were

717 classified by projection as described above, using the modified procedure for inDrop data.

718 TCGA LUAD RNAseq data was downloaded using the GDCquery and GDCdownload

719 functions from the TCGAbiolinks R package. GDCquery options included project="TCGA-

720 LUAD", data.category="Transcriptome Profiling", data.type="Gene Expression

721 Quantification", workflow.type="HTSeq - FPKM", experimental.strategy="RNA-Seq", and

722 legacy $=F$. Whole exome sequencing data was downloaded using the GDCquery_Maf function

723 with arguments tumor="TCGA-LUAD" and pipelines="mutect 2 ". Clinical data was downloaded

724 using the GDCquery_clinic function with arguments project="TCGA-LUAD" and

725 type="clinical".

726 Processed CPTAC lung adenocarcinoma data was downloaded from the CPTAC Data

727 Portal https://cptac-data-portal.georgetown.edu/cptacPublic/.

728

729 Determination of sample-sample distances

730 Sample-sample distances were computed as the Euclidean distance between vectors consisting of

731 the $\log _{10}$-transformed cell type frequencies, where frequencies were computed as a fraction of

732 total immune cells. A regularization factor of $10^{-3}$ was applied prior to applying the log-transform. 


\section{Determination of myeloid cell type-specific gene scores}

735 Lists of mutually-exclusive genes were used to compare monocytes, cDC2, and M $\Phi$ in Figure 2 ,

736 and monocytes, $\mathrm{AM} \Phi$, and MoMФ in Figure 3. For these analyses, genes were identified as

737 "mutually exclusive" if the average expression was at least $2 \mathrm{x}$ greater in a given population than

738 in the other comparison populations. To account for the large diversity of MoMФ clusters, the

739 maximum average expression of each MoМФ subtype was used instead of the overall average

740 expression. Resulting gene lists are presented in Table S4. Cells were scored according to the

741 resulting gene lists as the Log-transformed fraction of UMI belonging to the gene list. Histograms

742 were generated with the R function density using default parameters.

\section{Modules analyses}

745 Gene-gene correlation modules were generated using a similar method to that previously 746 described. Briefly, cells were downsampled to 2000 UMI prior to selecting a set of variable genes,

747 similar to the selection of genes in preparation for seeding the clustering ${ }^{16}$. The gene-gene

748 correlation matrix for this gene set was then computed for each sample over the cell population of

749 interest. Correlation matrices were averaged following a Fisher Z-transformation. Applying the

750 inverse transformation then resulted in the best-estimate correlation coefficients of gene-gene

751 interactions across the dataset. Genes were clustered into modules using complete linkage

752 hierarchical clustering over correlation distance. Histograms of module expression scores were

753 generated with the R function density using default parameters.

754

755 Classification of CD4+ versus CD8+ Tactivated $_{\text {cells }}$ 
756 CITEseq staining on a subset of patients was used to build a gene-set-based classifier that could

757 use mRNA UMI data to discriminate CD4+ versus CD8+ cells within the $\mathrm{T}_{\text {activated }}$ cluster. To

758 identify these gene sets, cells from 2 patients used as a training set were gated based on a $\log _{2} \mathrm{FC}$

759 of raw ADT counts of raw CD4/CD8 $>1$ and compared by differential expression. Genes were

760 filtered by expression $>10^{-4}$ and $\log _{2} \mathrm{FC}>1$, and nonspecific or noise-related genes such as

761 those associated with cell-cycle, long-non-coding RNAs, heat shock proteins, immunoglobulin

762 genes, ribosomal proteins, XIST, and histone transcripts. Resulting gene lists are reported in Table

763 S4. Cells were scored based on the fraction of RNAs belonging to the resulting gene lists, and a

764 discrimination threshold for the ratio of the CD4 vs. CD8 gene lists was determined based on the

765 overall accuracy in discriminating between CITEseq-defined CD4+ vs. CD8+ cells in the training

766 set. This gene score discriminator was validated using cells from a test set comprised of cells from

7674 additional patients analyzed by CITEseq $(584,593,596,630)$, and on cells with unique detection

768 of either $C D 4$ or at least one of $(C D 8 A, C D 8 B)$.

769

\section{$770 \quad$ Analysis of cycling $\mathbf{T}$ cell cluster}

771 To analyze the phenotypic makeup of the cluster of $\mathrm{T}$ cells expressing cell-cycle genes, we

772 generated gene sets based on the other T cell phenotypes described here to score each cell within

773 the cluster. To do this, we pooled the cells of each other T cell phenotype to compute its average

774 expression. We then identified a gene list for each phenotype defined by expression $>1 \mathrm{e}-5$ and

$775 \log 2 \mathrm{FC}>0.25$ compared to the maximum of the other phenotypes. From this list, we excluded

776 variable TCR genes, and other genes associated with noise or cell stress. The gene lists for the

$777 \mathrm{~T}_{\text {naive/CM-like }}$ cell types were grouped, since these phenotypes were very similar. Resulting gene lists

778 are reported in Table S4. 
For each cell in the cycling cluster, we then computed the fraction of UMI belonging to

780 each gene signature after removing UMIs belonging to the list of genes associated with the cycling

781 cluster that was calculated as above. We performed spherical k-means clustering using the function

782 skmeans() in the skmeans R package on these signature fractions in order to group cells within the

783 cycling cluster according to phenotypic subtype by spherical k-means cluster.

784

785 Single-cell TCRseq analysis

786 Single T cells were grouped by clonotype according to their precise combination of $\alpha$ and $\beta$ chains

787 present (uniquely defined by CDR3 sequence and V, D, and J gene usage), with the following

788 acceptations in order to filter for high quality singlets:

789 1. Cells with contigs encoding $>3$ productive $\alpha$ and $\beta$ chains were excluded as multiplets.

2. Cells with contigs encoding $>3$ productive $\alpha$ and $\beta$ chains that completely overlapped

791 with observed cells within the multiplets were also excluded as multiplets.

792 3. Remaining cells with 3 unique $\alpha$ and $\beta$ chains that could be uniquely associated with

793 similar cells displaying 2 unique $\alpha$ and $\beta$ chains were assumed to be clonally related, whereas cells

794 that could be similarly associated with multiple distinct sets of cells expressing 2 unique $\alpha$ and $\beta$

795 chains were excluded as doublets.

796 4. Cells in which a single TCR chain was observed were assumed to be clonally related to

797 any cells with 2 unique $\alpha$ and $\beta$ chains to which they uniquely associated.

798 5. Remaining cells in which a single TCR chain was observed were excluded if they 799 matched ambiguously to multiple cells with 2- or 3-chains.

800 Clonality scores were computed for each T cell type in each patient as 1-Peilou's eveness 801 over the set of unique TCRs as previously described ${ }^{52}$. 


\section{Ligand-receptor analysis}

804 Ligand-receptor intensity scores for a set of secreted ligands ( $\operatorname{ref}\left({ }^{33}\right)$ and Table S6) were calculated 805 as previously reported ${ }^{16}$. Briefly, for each ligand-receptor interaction, for each source cell type and each receiver cell type, the intensity score was equal to the product of ligand generation from the

807 source cell type relative to the total RNA with the expression of the receptor on the receiver cell 808 type. Scores were independently calculated for $\mathrm{LCAM}^{\mathrm{hi}}$ and $\mathrm{LCAM}^{\mathrm{lo}}$ patient sets in nLung and

809 Tumor tissues. To determine these patient sets, patients were sorted by the geometric mean of 810 lineage-normalized cellular frequencies of LCAM ${ }^{\text {hi }}$ and LCAM $^{\text {lo }}$ cell types, and the top half of 811 patients were defined as LCAM $^{\text {hi }}$ with the bottom half defined as LCAM ${ }^{\text {lo }}$. Only patients analyzed 812 using 10X Chromium V2 with immune cells purified with magnetic beads were used for this 813 analysis. The patients included in these groups were: LCAM ${ }^{\text {hi }}:(408,403,522,371,570,714,584$, $814377,406,564,630,578,514)$; LAM $^{\text {lo }}:(571,596,393,593,626,378,370,410,572,558,581$, $815596,729)$.

\section{Identification of LCAM ${ }^{\text {hi }}$ and LCAM ${ }^{\text {lo }}$ bulk-RNA gene signatures}

818 To define genes that could probe the presence of $\mathrm{LCAM}^{\mathrm{hi}}$ or $\mathrm{LCAM}^{\mathrm{lo}}$ cell types in bulk RNA 819 data, we adopted a similar strategy to that used previously for the projection of bulk data onto 820 signatures defined by cellular axes as measured with scRNA ${ }^{16,34}$. Cells were evenly sampled from

$821 \mathrm{LCAM}^{\mathrm{hi}}$ and LCAM ${ }^{\mathrm{lo}}$ patients (1409 cells per patient), and sampled cells were then pooled within 822 the groups. Differentially expressed genes $\left(F D R<10^{-3}\right.$ and $\left.\log 2 \mathrm{FC}>1\right)$ were retained. Genes that 823 were expressed in the filtered epithelial cells $>2 x$ higher than in immune cells on average were

824 removed. Among the remaining differentially expressed genes, those that were expressed on 
825 average within any $\mathrm{LCAM}^{\mathrm{hi}}$ or $\mathrm{LCAM}^{\mathrm{lo}}$ subtype with $\log 2 \mathrm{FC}>3$ compared to the highest

826 expressing subtype in the opposite group were retained. These gene lists were further abbreviated

827 to include no more than 10 genes per cell type, in order to balance the number of genes coming

828 from any individual cell type. In order to increase the differential expression effect sizes observed,

829 only the most extreme $6 \mathrm{LCAM}^{\mathrm{hi}}$ and $\mathrm{LCAM}^{\mathrm{lo}}$ patients processed with CD45+ bead enrichment

830 and 10X Chromium V2 were included in the differential expression analysis. These patients were

831 LCAM ${ }^{\mathrm{hi}}:(408,403,714,522,371,570)$, and $\operatorname{LCAM}^{\mathrm{lo}}:(571,596,393,593,626,378)$.

833 Calculation of LCAM ${ }^{\text {hi }}$, LCAM ${ }^{\mathrm{lo}}$, and ensemble LCAM scores in bulk-RNA datasets

834 Bulk RNA expression datasets were log-transformed and z-scored. For each cell type associated 835 with $\mathrm{LCAM}^{\mathrm{hi}}$ or $\mathrm{LCAM}^{\mathrm{lo}}$, the resulting $\mathrm{z}$-scores of the associated genes were averaged and z-

836 scored. A summary average of these values was then computed across all the cell types associated

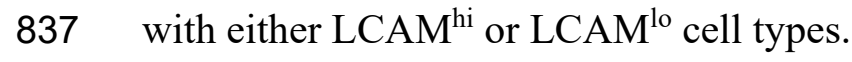

\section{Published statistics for TCGA Lung adenocarcinoma patients}

840 Estimates of total immune content present in each TCGA sample (ESTIMATE score) ${ }^{35}$ were

841 download from https://bioinformatics.mdanderson.org/public-software/estimate/.

842 Scores associating mutational signatures $^{41}$ with individual TCGA samples were 843 downloaded from the mSignatureDB ${ }^{42}$ website http://tardis.cgu.edu.tw/msignaturedb/. For the present 844 study, detection of Signature 4 was used to indicate presence of smoking-related mutations.

845 Counts of Indel Neoantigens, SNV Neoantigens, and CTA score in TCGA cases were 846 accessed from Table S1 of ref. $\left({ }^{53}\right)$.

\section{Generation of stromal cell type scores}


849 Fibroblast and endothelial cell count matrices from tumor and nLung of 8 NSCLC patients ${ }^{5}$ were

850 downloaded

from

851 https://gbiomed.kuleuven.be/english/research/50000622/laboratories/54213024/scRNAseq-

852 NSCLC. Previously-applied ${ }^{5}$ cluster annotations were assumed, where endothelial cluster 6 was

853 defined as "lymphatics", and endothelial and fibroblast clusters were defined based on enrichment

854 in tumor or nLung: endothelial clusters 3 and 4 were pooled as "Tumor BEC", endothelial clusters

8551 and 5 were pooled as "Normal BEC", fibroblast cluster 1 was defined as "Normal fibroblast",

856 and fibroblast clusters 1, 2, 3, 4, 5, and 7 were pooled as "CAF". For each of these cell types, gene

857 scores were defined based on a minimum average expression of $10^{-4}$ and a minimum fold-change

858 threshold of 4 compared to any other stromal cell type. Cell type gene-scores were defined in

859 TCGA lung adenocarcinoma using the average z-scored gene expression of each stromal gene list.

861 DATA AVAILABILITY

862 Human scRNAseq, TCRseq, and CITEseq data is available at GEO accession GSE154826.

\section{ACKNOWLEDGMENTS}

865 This work was supported by National Institutes of Health (NIH) grants 5T32CA078207 (to

866 A.M.L.). We thank A. Magen, P. Hamon, M. Casanova-Acebes for critical comments on the

867 manuscript; and the Mount Sinai flow cytometry core, Human Immune Monitoring Center and

868 Mount Sinai Biorepository for support. Research reported in this paper was supported by the Office

869 of Research Infrastructure of the National Institutes of Health under award numbers

870 S10OD018522 and S10OD026880. The content is solely the responsibility of the authors and does

871 not necessarily represent the official views of the National Institutes of Health. Data used in this 
872 publication were generated by the TCGA Research Network, and the National Cancer Institute

873 Clinical Proteomic Tumor Analysis Consortium (CPTAC). Research support was provided by

874 Regeneron and Takeda. We recognize the patients and their families for their important

875 contributions and sacrifices.

876

\section{AUTHOR CONTRIBUTIONS}

878 MM and EK conceived the project. AML, AR, and MM designed the experiments. AML, EK, and

879 MM wrote the manuscript. AML, EK, and MD performed computational analysis. TM, MB, AW, 880 and RF facilitated access to human samples. AML, JG, CC, BM, AT, LW, JL, NM, GM, and KT

881 performed experiments. NRD and GT funded part of the study. AL conducted patient consents and 882 facilitated regulatory items. JG, JM, GM, ZZ, FP, RS, AK, PW, HS, and TM provided further 883 intellectual input.

\section{DECLARATION OF INTERESTS}

886 Research support for this work was provided by Regeneron and Takeda. The authors declare no 887 other competing financial interests. 
FIGURE LEGENDS

890

Figure 1. scRNA- and CITE-seq establish the diversity of transcriptional states in the tumor microenvironment.

A, Study overview. Resected specimens of tumor tissue and non-involved lung (nLung) were

894 digested to single cell suspensions, enriched for CD45+ cells, and subjected to single cell assays including CITEseq and TCRseq.

B, Clinical data of patients undergoing resection indicating summary pathological stage, smoking

897 history, histological diagnosis, and sex.

898 C, Expression of cell type marker genes across scRNAseq clusters of immune cells, grouped by

899 lineage annotation (MNP: mononuclear phagocyte; pDC: plasmacytoid dendritic cell). Heatmap

900 shows the number of unique molecular identifiers (UMI) per cell. Clusters are shown using an

901 even number of randomly selected cells from 7 matched tumor and nLung sample pairs who were

902 analyzed by CITEseq. Cells were downsampled to $2000 \mathrm{UMI} /$ cell.

903 D, Expression of lineage-defining surface markers on single cells, as measured by CITEseq. Single

904 cells correspond directly to cells shown in (C). CITEseq count values were first quantile

905 normalized across patients, then row-normalized across cells in the heatmap.

906 E, Cells per cluster as a percent of total immune cells across 35 tumor and 32 matched nLung 907 samples. Clusters correspond directly to those shown in (C) and (D).

908 F, Box-plots of Euclidean distances between pairs of samples among nLung only (nLung-nLung),

909 tumor only (tumor-tumor), or between nLung samples and tumor samples (Tumor-nLung).

910 Distances between pairs of patient-matched samples were excluded from the Tumor-nLung 
911 distribution to prevent confounding due to patient-specific effects. $* * * \mathrm{P}<0.001$, Wilcoxon rank-

912 sum test.

913 G, Log-ratios between cell type frequencies in tumor and nLung. Clusters were grouped by cell

914 type annotation. Crosses represent error bars showing the mean \pm SEM of $\log _{2} \mathrm{FC}$ estimates of

915 differences in cell type frequency between tumor and nLung using the cohort collected in the 916 present study (Mount Sinai; $\mathrm{x}$-axis) or the cohort in ref. ${ }^{5}$ (y-axis).

918 Figure 2. Intratumoral DC comprise expanded MoDC and express an LCH-like signature.

919 A, Expression of key genes discriminating scRNAseq clusters of DC, grouped by cell type 920 annotation (MoDC: monocyte-derived DC; cDC: classical DC). Heatmap shows the number of 921 UMI per cell. Clusters are shown using an even number of randomly selected cells from each, 922 drawing from patients who were analyzed by CITEseq with the DC panel shown in (B) (4 matched 923 tumor-nLung tissue pairs). Cells were downsampled to $2000 \mathrm{UMI} /$ cell.

924 B, Expression of DC surface markers on single cells, as measured by CITEseq. Single cells 925 correspond directly to cells shown in (A). CITEseq count values were first quantile normalized 926 across patients, then row-normalized across cells in the heatmap.

927 C, Differences between tumor and nLung of DC frequencies normalized to total $\mathrm{DC} ; * \mathrm{P}<0.05$, $928 * * \mathrm{P}<0.01, * * * \mathrm{P}, 0.001$ (Wilcoxon signed-rank test with Bonferroni correction; $\mathrm{N}=25$ matched 929 tissue pairs with $>50$ DC observed in each tissue).

930 D, Barplots showing average expression of LAMP3 (DC-LAMP) and CD274 (PD-L1) across DC 931 clusters.

932 E, MICSSS imaging showing spatial distribution of DC-LAMP+/PD-L1+ DC in proximity to T 933 cells in a TLS. 
934 F, Expression of follicular dendritic cell marker MYH11 in TLS in an adjacent section to that

935 shown in $(\mathbf{E})$.

936 G, Expression among CD14+ monocytes and DC of monocyte, cDC2, and M $\Phi$ cell type specific

937 gene signatures (See Figure S2D, E). Heatmaps show expression of 20 genes from each score

938 among single-cells evenly sampled by cell type (left) and as corresponding summary scores. Cells

939 were ordered by the ratio of monocyte:cDC2 summary scores and were downsampled to 2000

940 UMI.

$941 \mathbf{H}$, Boxplots showing average expression of LCH-like signature genes across DC populations in

942 distinct nLung and tumor samples.

944 Figure 3. Tumors exclude AMФ and exhibit a diversity of MoMФ populations.

945 A, Average cluster expression of lineage-defining monocyte and M $\Phi$ clusters based on literature

946 review, grouped by cell type annotation.

947 B, Expression of myeloid surface markers on single cells, as measured by CITEseq. Clusters are

948 shown using an even number of randomly selected cells from each, from patients who were

949 analyzed with the panel shown (4 matched tumor-nLung pairs). CITEseq count values were first

950 quantile normalized across patients, then row-normalized across cells in the heatmap.

951 C, Histograms of gene module scores per cell type (see also Figure S3D-J).

952 D-F, Expression among CD14+ monocytes, MoMФ, and AMФ of cell type specific gene scores.

953 Gene scores were generated based on sets of mutually exclusive, differentially expressed genes

954 among $\mathrm{AM} \Phi, \mathrm{MoM} \Phi$, and CD14+ monocytes (see Figure S3I). Cells are plotted by AM $\Phi$ and

955 MoМФ score, and cell-annotations are indicated by colored dots or contour plots (D). Cells are 
956 plotted on similar axes and colored by CD14+ monocyte score (E), or by expression of individual 957 genes $(\mathbf{F})$.

958 G, Differences between tumor and nLung of lineage-normalized monocyte and M $\Phi$ frequencies;

$959 * \mathrm{P}<0.05 ; * * \mathrm{P}<0.01, * * * \mathrm{P}, 0.001$ (Wilcoxon signed-rank test with Bonferroni correction; $\mathrm{N}=32$

960 matched tissue pairs).

$961 \mathbf{H}$, Average cell type expression of secreted factors across MNP cell types.

\section{Figure 4. CITEseq and TCR analysis of the adaptive immune compartment.}

964 A, Expression of key genes discriminating scRNAseq clusters of T cells, grouped by cell type 965 annotation. Heatmap shows the number of UMI per cell. Clusters are shown using an even number 966 of randomly selected cells from each, drawing from patients who were analyzed by CITEseq with

967 the T cell panel shown in (B) (2 matched tumor-nLung tissue pairs). Cells were downsampled to $9682000 \mathrm{UMI} / \mathrm{cell}$.

969 B, Expression of T cell surface markers on single cells, as measured by CITEseq. Single cells 970 correspond directly to cells shown in (A). CITEseq count values were first quantile normalized 971 across patients, then row-normalized across cells in the heatmap.

972 C, Differences between tumor and nLung of population frequencies normalized by total NK and

$973 \mathrm{~T}$ cells; $* \mathrm{P}<0.05 ; * * \mathrm{P}<0.01, * * * \mathrm{P}<0.001$ (Wilcoxon signed-rank test with Bonferroni correction, $974 \quad \mathrm{~N}=32$ matched tissue pairs).

975 D, E, Phenotypic distribution of T cells among tissue-stratified clonotypes. Frequencies of unique 976 TCRs observed by scTCRseq in nLung (x-axis) or tumor in a representative patient (D). In (E), 977 cells were first grouped by TCR tissue tropism categories as defined in (D); for 3 patients, the 978 phenotypic makeup of the cells with unique TCRs, tissue-specific TCRs, or TCRs shared across 
979 tissues is plotted for nLung (i) and tumor tissues (ii) is plotted as a percent of cells with similarly

980 tissue-distributed TCRs. Each patient is indicated by shape.

981 F, Expression of key genes discriminating scRNAseq clusters of B and plasma cells, grouped by

982 cell type annotation. Heatmap shows the number of UMI per cell. Clusters are shown using an

983 even number of randomly selected cells from each, drawing from patients who were analyzed by

984 CITEseq with the B cell panel shown in (G) (4 matched tumor-nLung tissue pairs). Cells were

985 downsampled to $2000 \mathrm{UMI} / \mathrm{cell}$.

986 G, Expression of B and plasma cell surface markers on single cells, as measured by CITEseq.

987 Single cells correspond directly to cells shown in (F). CITEseq count values were first quantile

988 normalized across patients, then row-normalized across cells in the heatmap.

Figure 5. Cell-cell interactions drive an axis of adaptive activation.

991 A, Spearman correlation of cell type frequencies after normalization within lineage. Analysis

992 includes 23 tumors that were processed similarly using 10X Chromium V2 and CD45+ magnetic

993 bead enrichment.

994 B, Lineage-normalized cell type frequencies of $\mathrm{LCAM}^{\mathrm{hi}}$ and $\mathrm{LCAM}^{\mathrm{lo}}$ cell types among pooled 995 nLung and Tumor samples from Mount Sinai and refs. $\left({ }^{5,6}\right)(50$ tumor patients with 40 matched 996 nLung samples). nLung samples are ordered to match the order of tumor samples based on 997 frequencies of LCAM celltypes.

998 C, Immune lineage frequencies of nLung and Tumor samples; with columns corresponding to 999 patient ordering in (B).

1000 D-I, Log2 Ratio of ligand-receptor (LR) intensity scores between tumor and nLung of LCAM ${ }^{\text {hi }}$ 1001 patients, ("LR ratio"; y-axis) and LCAM ${ }^{\text {lo }}$ patients (x-axis). All interactions among T cells, B cells, 
$\mathrm{M} \Phi, \mathrm{MoDC}, \mathrm{cDC}$, and monocytes, colored by indication of significance (permutation test, D).

1003 Dashed diagonal line indicates unity.

1004 E-I, Showing same data as in (D), but highlighting in bold LR ratios for interactions between T 1005 cell ligands and B cell receptors $(\mathbf{E}), \mathrm{T}$ cell ligands and cDC receptors $(\mathbf{F}), \mathrm{M} \Phi$ ligands and T cell receptors $(\mathbf{G})$, MoDC ligands and T cell receptors $(\mathbf{H})$, and $\mathrm{cDC}$ ligands and T cell receptors $(\mathbf{I})$.

1007 Labelled interactions are plotted in red.

1008

Figure 6. Tumor features related to the LCAM immune response.

1010 A, Normalized expression of $\mathrm{LCAM}^{\mathrm{hi}}$ and $\mathrm{LCAM}^{\mathrm{lo}}$ bulk-RNA signature genes, determined as

1011 shown in Figure S6A, B and as described in the methods, in TCGA lung adenocarcinoma

1012 dataset. Cell type association with sets of genes for each signature is shown. Patients are sorted

1013 along y-axis by ensemble LCAM score.

1014 B-D, Scatter plots of the ensemble LCAM score (y-axis) with signature scores based on genes

1015 that are specific for CAFs (B), normal fibroblasts (C), or the difference between these scores (D)

1016 in TCGA lung adenocarcinoma data. Stromal signatures are based on the stromal data reported in 1017 ref. ${ }^{5}$

1018 E, Scatter plot of LogTMB and ensemble LCAM score. Patients are divided into those with 1019 presence of a smoking-related mutational signature (black) and those without presence of the 1020 signature (red). Black and red lines indicate linear regression relationships computed over each 1021 group of patients independently $(\mathrm{r}=0.38 ; \mathrm{p}=9.2 \mathrm{e}-5$ in the undetected smoking signature group; $1022 \mathrm{r}=0.34 ; \mathrm{p}=1.1 \mathrm{e}-12$ in the detected signature group).

1023 F, Scatter plot of Cancer testes antigen expression score (CTA score), as computed in ref. ${ }^{53}$, and 1024 the residuals of the regression of the ensemble LCAM score on the LogTMB. 
$1025 \mathbf{G}$ and $\mathbf{H}$, Boxplots showing either the ensemble LCAM score $(\mathbf{G})$, or TMB $(\mathbf{H})$ among TCGA

1026 lung adenocarcinoma patients, divided by combinations of driver mutations.

1027 I and $\mathbf{J}$, Histograms of residuals of the regression of the ensemble LCAM score on the LogTMB, 1028 with patients stratified by TP53 (I) or KRAS (J) mutational status (Two-sided t-test).

1030 Figure S1. Integration of scRNA samples and datasets for common cell type analysis.

1031 A, Comparison of per-sample estimated noise levels in the training set of cells used for clustering 1032 and model formation ( $\mathrm{x}$-axis) compared to the per-sample estimated noise in a withheld test set of 1033 cells that were mapped to the model clusters by probabilistic projection.

1034 B and C, Illustration of how incorporating a fit noise component improves the concordance 1035 between predicted expression and of cells mapped to the $\mathrm{T}_{\text {reg }}$ cluster and observed expression. $\mathrm{Y}$ 1036 axis shows the predicted expression of $\mathrm{T}_{\text {regs }}$ in individual samples without accounting for noise (B) 1037 or accounting for noise (C), against the observed average expression (X-axis). Genes were color1038 coded by the ratio between the observed expression and the model without accounting for noise. 1039 Estimation of the noise component is detailed in the methods.

1040 D, Per-sample estimated noise levels in 10X chromium V2 samples that were used for clustering, 1041 10X chromium V2 samples that were analyzed by projection onto the clustering model and not 1042 used in the clustering, 10X chromium 5' samples that were analyzed by projection, and samples 1043 from external datasets ${ }^{5,6}$ that were analyzed by projection.

1044 E, Boxplots showing the distribution of UMI per cell in each cluster.

1045 F, Barplots showing number of cells in Mount Sinai dataset mapping to each cluster. 
1046 G, Heatmap showing row-normalized cell type frequencies in a public dataset ${ }^{5}$ with samples 1047 spanning 3 regions each in a cohort of 8 NSCLC patients. Samples are clustered by spearman 1048 correlation distance. Sample names are colored by patient.

$1049 \mathbf{H}$, Box plots of Euclidean distances based on log-transformed cluster frequencies between samples 1050 of different patients or from the same patient, as in $(\mathbf{G})$, from ref. ${ }^{5}$. $* * * \mathrm{P}<0.001$, Wilcoxon rank1051 sum test.

\section{Figure S2. Module analysis of DC.}

1054 A, Barplots showing total number of cells mapped to each individual DC cluster in the Mount 1055 Sinai cohort.

1056 B, Boxplots showing number of cells mapped to each individual DC cluster per tumor sample in 1057 the Mount Sinai cohort.

1058 C, Differences between tumor and nLung of DC frequencies normalized to total MNP; $* \mathrm{P}<0.05$, $1059 * * \mathrm{P}<0.01, * * * \mathrm{P}, 0.001$ (Wilcoxon signed-rank test with Bonferroni correction; $\mathrm{N}=26$ matched 1060 tissue pairs with $>250$ MNP observed in each tissue).

1061 D, $\log _{2} \mathrm{FC}$ and expression level distributions of gene sets that are mutually exclusively expressed 1062 in CD14+ monocytes, MФ, and cDC2 (See Figure 2G).

1063 E. Histograms of cDC2 and MФ scores, using gene lists generated as shown in (D).

1064 F-I, Gene module analysis of DC clusters. Correlation of gene module expression across all DC 1065 (F), five example genes from each module, ranked by correlation to the other genes in the module 1066 and colored by total expression in DC $(\mathbf{G})$, boxplots showing $\log _{2} \mathrm{FC}$ of module expression among 1067 all DC between patient matched tumor and nLung samples $(\mathbf{H})$, and normalized average cluster 1068 expression of modules (I). 
J, Boxplots showing expression of LCH-like signature genes across DC populations in distinct

1070 nLung and tumor samples from ref. ${ }^{5}$.

\section{Figure S3. Diversity of nlung and tumor-infiltrating МФ populations.}

1073 A, Expression of key genes discriminating scRNAseq clusters of monocytes and MФ, grouped by 1074 cell type annotation. Heatmap shows the number of UMI per cell. Clusters are shown using an 1075 even number of randomly selected cells from each, drawing from 35 tumor and 32 nLung samples. 1076 Cells were downsampled to $2000 \mathrm{UMI} / \mathrm{cell}$.

1077 B, Scatter plots showing normalized CITEseq CD10 and CD206 surface marker counts on AMФ, $1078 \mathrm{MoM} \Phi$, and CD14+ monocytes in nLung of a representative patient.

1079 C, IHC of CD10 staining AM $\Phi$ in the airspaces of nLung tissue.

1080 D-G, Gene module analysis of monocyte and MФ clusters. Correlation of gene module expression 1081 across all monocytes and MФ (D). Module groups illustrate groups of correlated modules which 1082 are expressed most specifically on $\mathrm{AM} \Phi$, MoMФ, and monocytes (see G). Five example genes 1083 from each module, ranked by correlation to the other genes in the module and colored by total 1084 expression in monocytes and $\mathrm{M} \Phi(\mathbf{E})$, boxplots showing $\log _{2} \mathrm{FC}$ of module expression among all 1085 monocytes and $\mathrm{M} \Phi$ between patient matched tumor and nLung samples $(\mathbf{F})$, and normalized 1086 average cluster expression of modules $(\mathbf{G})$.

$1087 \mathbf{H}, \log _{2} \mathrm{FC}$ and expression level of gene sets that are mutually exclusively expressed in CD14+ 1088 monocytes, АMФ, and MoMФ (See Figure 3D-F).

1089 
1091 A, Differential expression within the $\mathrm{T}_{\text {activated }}$ cluster of cells staining for CD4 versus CD8 by

1092 CITEseq (y-axis) vs. average $\mathrm{T}_{\text {activated }}$ expression (x-axis).

1093 B, Classification of $\mathrm{T}_{\text {activated }}$ cells as CD4+ or CD8+ based on the ratio of CD4-related or CD81094 related gene signatures learned from cells of 2 patients (training set; open circles) and validated on 1095 cells of 4 additional validation patients (test set; black dots). Red line indicates gene ratio threshold 1096 learned from the training set. Only cells where the CITEseq CD4:CD8 count ratio is $>2$ or $<1 / 2$ 1097 are considered.

1098 C, Validation of CD4/CD8 classification scheme shown in (B) for cells without CITEseq staining. 1099 Cells were considered to be CD4+ or CD8+ based on unique RNA detection of either CD4 (blue 1100 points) or at least one $C D 8 A$ or $C D 8 B$ transcript (green points). The discriminant line is equivalent 1101 to the gene ratio threshold learned from CITEseq data, shown in $(\mathbf{B})$.

1102 D, Expression of key genes in CD4-related and CD8-related gene signatures for discriminating 1103 CD4+ and CD8+ activated T cells. Cells are sorted by ratio of these gene signatures, and the line 1104 is drawn to indicate the cells discriminated based on the threshold in panel (B). Heatmap shows 1105 the number of UMI per cell. Cells represent $\mathrm{T}_{\text {activated }}$ cells from 35 tumors, and were downsampled 1106 to $2000 \mathrm{UMI} / \mathrm{cell}$.

1107 E, Frequency of CD8+ or CD4+ $\mathrm{T}_{\text {activated }}$ cells across 35 patients, as determined by gene signature 1108 scores learned from CITEseq (as in A-D).

1109 F-H, Spherical k-means sub-clustering on cell type scores of cells within the cycling T cell cluster 111018 based on gene scores generated from other T cell clusters. Heatmap of single-cell expression of 1111 cell type scores, grouped by sub-cluster $(\mathbf{F})$, number of cells in each sub-cluster (G; nLung shown 1112 in blue, tumor in brown; lines dividing bars horizontally discriminate groups of cells from distinct 
1113 patients), and the frequency of cycling T cells of each $\mathrm{T}$ cell phenotype (H; data points represent

1114 samples with at least 50 cells of the given phenotype).

1115 I, TCR clonality score of phenotypic groups in nLung (blue) and tumor (brown). Dots represent

1116 individual samples with at least 30 cells of indicated phenotype. $\mathrm{N}=3$ patients with tumor-nLung

1117 pairs.

$1118 \mathbf{J}$, Number of cells within each TCR category, determined as in Figure 5D, in matched nLung and 1119 tumor samples of 3 patients, each patient indicated by shape.

$1120 \mathbf{K}$, Number of unique TCRs represented in each TCR category, determined as in Figure 5D.

1121 L, Differences between tumor and nLung of lineage-normalized B and plasma cell type

1122 frequencies. All comparisons were not significant $(\mathrm{P}>0.05$, Wilcoxon signed-rank test, $\mathrm{N}=32$ 1123 matched tissue pairs).

\section{Figure S5. Ligand-receptor intaractions in LCAM ${ }^{\text {hi }}$ and LCAM LA $^{\text {lo }}$ tumors.}

1126 A, Lineage-normalized cell type frequencies of all cell types among pooled nLung and Tumor

1127 samples from Mount Sinai and refs. ${ }^{5,6}$ (50 tumor patients with 40 matched nLung samples).

1128 B and C, Column-normalized expression of highly expressed secreted ligands (B) and associated

1129 receptors $(\mathbf{C})$ across all immune cell types, connected by lines linking ligands to receptors.

1130 Connectors are colored by association with LCAM $^{\text {hi }}$ patients (purple), LCAM ${ }^{\text {lo }}$ patients (green), 1131 or all tumors (orange).

1132 D-F, Log2 Ratio of ligand-receptor (LR) intensity scores between tumor and nLung of LCAM ${ }^{\text {hi }}$ 1133 patients, ("LR ratio"; y-axis) and LCAM ${ }^{\mathrm{lo}}$ patients (x-axis) as in Figure 5D, highlighting in bold 1134 LR ratios for interactions between B cell ligands and T cell receptors $(\mathbf{D}), \mathrm{T}$ cell ligands and MoDC 1135 receptors $(\mathbf{E})$, and T cell ligands and $M \Phi$ receptors $(\mathbf{F})$. Labelled interactions are plotted in red. 
1137 Figure S6. Projection of bulk RNA samples onto signatures defined by the LCAM scRNA

1138 axis.

1139 A and B, Derivation of the LCAM $^{\text {hi }}$ and LCAM $^{\text {lo }}$ gene signatures for scoring bulk RNA samples.

1140 Identification of differentially-expressed genes between averaged scRNAseq samples of LCAM ${ }^{\text {hi }}$

1141 and $\mathrm{LCAM}^{\mathrm{lo}}$ patients (A), and identification of differentially expressed genes that are specific to

1142 genes in the LCAM ${ }^{\text {hi }}$ or LCAM ${ }^{\text {lo }}$ cell types $(\mathbf{B})$.

1143 C-E, Scatter plots of immune ESTIMATE score ${ }^{35}$ with the LCAM ${ }^{\text {hi }}$ signature score $(\mathbf{C})$, the

1144 LCAM $^{\mathrm{lo}}$ signature score (D), or the difference between the LCAM $^{\text {hi }}$ and LCAM ${ }^{\mathrm{lo}}$ signature scores

1145 (i.e. the ensemble LCAM score; E).

1146 F, Spearman correlation of the LCAM $^{\text {hi }}$ and LCAM $^{\text {lo }}$ signature scores among the deciles of 1147 immune content. Error bars represent the 95\% confidence interval around the estimate of the 1148 spearman correlation.

1149 G, Scatter plots of the LCAM ${ }^{\text {hi }}$ and LCAM ${ }^{\text {lo }}$ signature scores, showing the $1^{\text {st }}($ black$), 3^{\text {rd }}($ green), 1150 and $10^{\text {th }}$ (red) deciles of immune content. Labelled trend lines are shown for other deciles.

$1151 \mathbf{H}$, Normalized expression of $\mathrm{LCAM}^{\mathrm{hi}}$ and $\mathrm{LCAM}^{\mathrm{lo}}$ bulk-RNA signature genes in the CPTAC lung 1152 adenocarcinoma dataset.

1153 I, Scatter plots of the ensemble LCAM score (y-axis) with signature scores based on genes that 1154 are specific for tumor blood endothelial cells (BEC; left), normal BEC (center), and lymphatic 1155 endothelial cells. Stromal signatures are based on the stromal data reported in ref. 5.

$1156 \mathbf{J}$, Boxplots showing ensemble LCAM scores among TCGA lung adenocarcinoma patients by 1157 TNM T-stage. 
$\mathbf{K}$, Scatter plot of LogTMB and ensemble LCAM score in CTPAC lung adenocarcinoma patients, 1159 with linear regression line.

$\mathbf{L}$ and $\mathbf{M}$, Scatter plots of the LogTMB and immune ESTIMATE $\operatorname{score}^{35}(\mathbf{L})$ and the T-cell 1161 inflamed gene expression profile $\left(\mathrm{GEP}^{39,40} ; \mathbf{M}\right)$ in TCGA lung adenocarcinoma patients.

$1162 \mathbf{N}$, Correlation between individual genes comprising the LCAM $^{\text {hi }}$ and LCAM ${ }^{\text {lo }}$ bulk gene 1163 signatures and LogTMB in TCGA lung adenocarcinoma patients.

$1164 \mathbf{O}$, Scatter plots of LogTMB and the LCAM ensemble score for patients by T-stage.

$1165 \mathbf{P}$ and $\mathbf{Q}$, Scatter plots of the number of indel-induced neoantigens $(\mathbf{P})$ and SNV-induced 1166 neoantigens $(\mathbf{Q})$ as computed in ref. ${ }^{53}$, and the residuals of the regression of the ensemble LCAM 1167 score on the LogTMB.

$1168 \mathbf{R}$ and $\mathbf{S}$, Boxplots showing either the ensemble LCAM score $(\mathbf{G})$, or TMB $(\mathbf{H})$ among CPTAC 1169 lung adenocarcinoma patients, divided by combinations of mutated driver mutations.

1170 T and $\mathbf{U}$, Histograms of residuals of the regression of the ensemble LCAM score on the LogTMB, 1171 with patients stratified by $\operatorname{STK} 11$ (T) or EGFR (U) mutational status (Two-sided t-test).

\section{SUPPLEMENTAL TABLES}

1174 Table S1. Sample table, with information about patient, tissue, 10X loading, and QC metrics

1175 Table S2. CITEseq panels used

1176 Table S3. QC table of GEX, HTO, and ADT libraries

1177 Table S4. Gene lists used in paper

1178 Table S5. Gene modules

1179 Table S6. Ligand-receptor pairs used in the analysis

1180 Table S7. Ligand-receptor statistics 


\section{REFERENCES}

1183 1. Siegel, R.L., Miller, K.D. \& Jemal, A. Cancer statistics, 2019. CA Cancer J Clin 69, 7-34 (2019).

1184 2. Travis, W.D., Brambilla, E., Burke, A.P., Marx, A. \& Nicholson, A.G. Introduction to The 2015 World Health Organization Classification of Tumors of the Lung, Pleura, Thymus, and Heart. $J$ Thorac Oncol 10, 1240-1242 (2015).

3. Remon, J., et al. Immune Checkpoint Inhibitors in Thoracic Malignancies: Review of the Existing

4. Laughney, A.M., et al. Regenerative lineages and immune-mediated pruning in lung cancer metastasis. Nat Med 26, 259-269 (2020).

5. Lambrechts, D., et al. Phenotype molding of stromal cells in the lung tumor microenvironment. Nat Med 24, 1277-1289 (2018).

6. Zilionis, R., et al. Single-Cell Transcriptomics of Human and Mouse Lung Cancers Reveals Conserved Myeloid Populations across Individuals and Species. Immunity 50, 1317-1334 e1310 (2019).

7. Lavin, Y., et al. Innate Immune Landscape in Early Lung Adenocarcinoma by Paired Single-Cell Analyses. Cell 169, 750-765.e717 (2017).

8. Kargl, J., et al. Neutrophils dominate the immune cell composition in non-small cell lung cancer. Nature Communications 8, 14381 (2017).

9. Guo, X., et al. Global characterization of $\mathrm{T}$ cells in non-small-cell lung cancer by single-cell sequencing. Nature Medicine 24, 978-985 (2018).

10. Thommen, D.S., et al. A transcriptionally and functionally distinct PD-1+ CD8+ T cell pool with predictive potential in non-small-cell lung cancer treated with PD-1 blockade. Nature Medicine 24,

11. Li, H., et al. Dysfunctional CD8 T Cells Form a Proliferative, Dynamically Regulated Compartment within Human Melanoma. Cell 176, 775-789 e718 (2019).

12. $\mathrm{Wu}, \mathrm{T} . \mathrm{D} .$, et al. Peripheral $\mathrm{T}$ cell expansion predicts tumour infiltration and clinical response. Nature 579, 274-278 (2020).

13. Binnewies, M., et al. Understanding the tumor immune microenvironment (TIME) for effective therapy. Nat Med 24, 541-550 (2018).

14. Rizvi, N.A., et al. Mutational landscape determines sensitivity to PD-1 blockade in non-small cell lung cancer. Science 348, 124 (2015).

15. Stoeckius, M., et al. Simultaneous epitope and transcriptome measurement in single cells. Nature methods 14, 865-868 (2017). 
16. Martin, J.C., et al. Single-Cell Analysis of Crohn's Disease Lesions Identifies a Pathogenic Cellular Module Associated with Resistance to Anti-TNF Therapy. Cell 178, 1493-1508.e1420 (2019).

1217 17. Rosenthal, R., et al. Neoantigen-directed immune escape in lung cancer evolution. Nature 567, 1218 479-485 (2019).

1219 18. Maier, B., et al. A conserved dendritic-cell regulatory program limits antitumour immunity. Nature

19. Zhang, Q., et al. Landscape and Dynamics of Single Immune Cells in Hepatocellular Carcinoma.

20. See, P., et al. Mapping the human DC lineage through the integration of high-dimensional

21. Dutertre, C.A., et al. Single-Cell Analysis of Human Mononuclear Phagocytes Reveals Subset-

22. Remark, R., et al. In-depth tissue profiling using multiplexed immunohistochemical consecutive Defining Markers and Identifies Circulating Inflammatory Dendritic Cells. Immunity 51, 573-589 e578 (2019).

23. Ioannidis, I. \& Laurini, J.A. Use of Smooth Muscle Myosin Heavy Chain as an Effective Marker

24. Allen, C.E., Merad, M. \& McClain, K.L. Langerhans-Cell Histiocytosis. New England Journal of Medicine 379, 856-868 (2018).

25. Senechal, B., et al. Expansion of regulatory T cells in patients with Langerhans cell histiocytosis.

26. Dudakov, J.A., Hanash, A.M. \& van den Brink, M.R. Interleukin-22: immunobiology and pathology. Annu Rev Immunol 33, 747-785 (2015).

27. Khosravi, N., et al. IL22 Promotes Kras Mutant Lung Cancer by Induction of a Pro-Tumor Immune

28. Chakarov, S., et al. Two distinct interstitial macrophage populations coexist across tissues in

29. Leach, S.M., et al. Human and mouse transcriptome profiling identifies cross-species homology in

30. Hashimoto, D., et al. Tissue-Resident Macrophages Self-Maintain Locally throughout Adult Life

31. Gibbings, S.L., et al. Three Unique Interstitial Macrophages in the Murine Lung at Steady State.

32. Singh, D., et al. CD4+ follicular helper-like T cells are key players in anti-tumor immunity. bioRxiv 1250 (2020). 
33. Ramilowski, J.A., et al. A draft network of ligand-receptor-mediated multicellular signalling in human. Nature Communications 6, 7866 (2015).

34. Neftel, C., et al. An Integrative Model of Cellular States, Plasticity, and Genetics for Glioblastoma.

35. Yoshihara, K., et al. Inferring tumour purity and stromal and immune cell admixture from

36. Salmon, H., Remark, R., Gnjatic, S. \& Merad, M. Host tissue determinants of tumour immunity.

37. Salmon, H., et al. Matrix architecture defines the preferential localization and migration of T cells

38. Samstein, R.M., et al. Tumor mutational load predicts survival after immunotherapy across multiple cancer types. Nat Genet 51, 202-206 (2019).

39. Ayers, M., et al. IFN- $\gamma$-related mRNA profile predicts clinical response to PD-1 blockade. The Journal of Clinical Investigation 127, 2930-2940 (2017).

40. Cristescu, R., et al. Pan-tumor genomic biomarkers for PD-1 checkpoint blockade-based

41. Alexandrov, L.B., et al. Mutational signatures associated with tobacco smoking in human cancer.

42. Huang, P.-J., et al. mSignatureDB: a database for deciphering mutational signatures in human

43. Dong, Z.-Y., et al. Potential Predictive Value of TP53 and KRAS Mutation Status for Response to

44. Biton, J., et al. TP53, STK11, and EGFR mutations predict tumor immune profile and the response

45. Rooney, Michael S., Shukla, Sachet A., Wu, Catherine J., Getz, G. \& Hacohen, N. Molecular and Genetic Properties of Tumors Associated with Local Immune Cytolytic Activity. Cell 160, 48-61 (2015).

46. Skoulidis, F., et al. STK11/LKB1 mutations and PD-1 inhibitor resistance in KRAS-mutant lung

47. Canon, J., et al. The clinical KRAS(G12C) inhibitor AMG 510 drives anti-tumour immunity. Nature 575, 217-223 (2019).

48. Stoeckius, M., et al. Cell Hashing with barcoded antibodies enables multiplexing and doublet detection for single cell genomics. Genome Biol 19, 224 (2018).

49. Paul, F., et al. Transcriptional Heterogeneity and Lineage Commitment in Myeloid Progenitors. Cell 163, 1663-1677 (2015). 
1287 50. Jaitin, D.A., et al. Massively parallel single-cell RNA-seq for marker-free decomposition of tissues $1288 \quad$ into cell types. Science 343, 776-779 (2014).

1289 51. Ding, J., et al. Systematic comparison of single-cell and single-nucleus RNA-sequencing methods. $1290 \quad$ Nature Biotechnology (2020).

1291 52. Kirsch, I., Vignali, M. \& Robins, H. T-cell receptor profiling in cancer. Mol Oncol 9, 2063-2070 $1292 \quad$ (2015).

1293 53. Thorsson, V., et al. The Immune Landscape of Cancer. Immunity 48, 812-830.e814 (2018). 


\section{bioRxiv preprint doi: https://doi.org/10.1101/2020.07.16.207605; this version posted July 22, 2020. The copyright holder for this preprint (which was not certified by peer review) is the author/funder. All rights reserved. No reuse allowed without permission.}

Figure 1

A

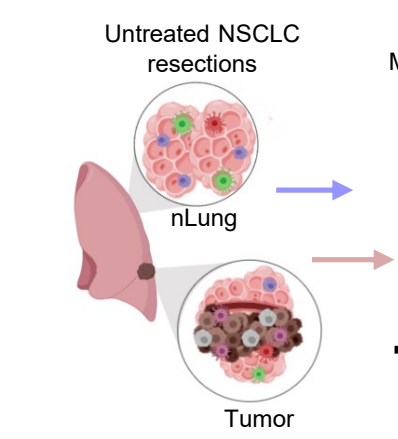

\section{Study overview}

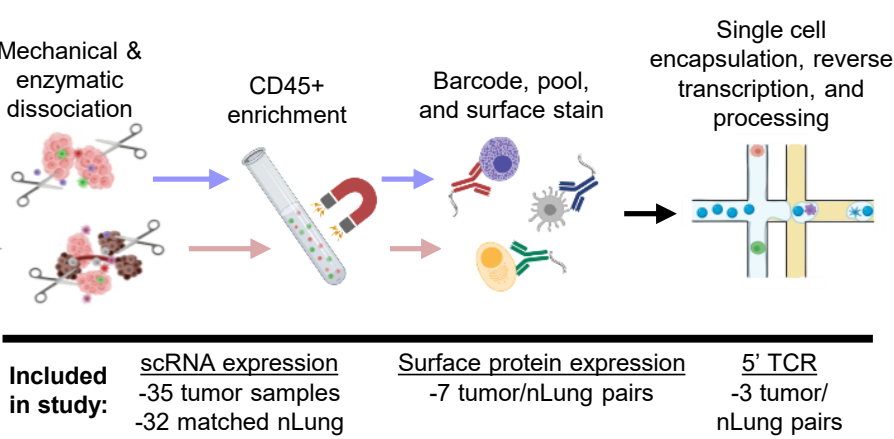

B Patient characteristics

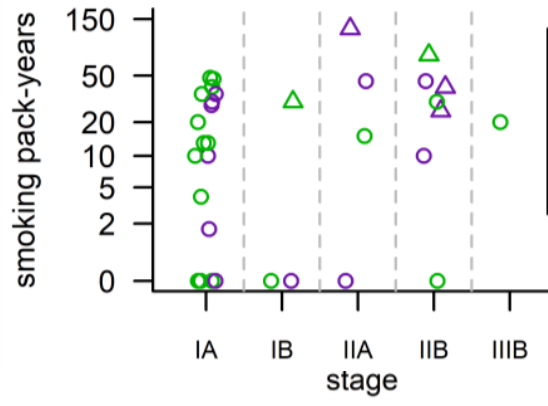

C

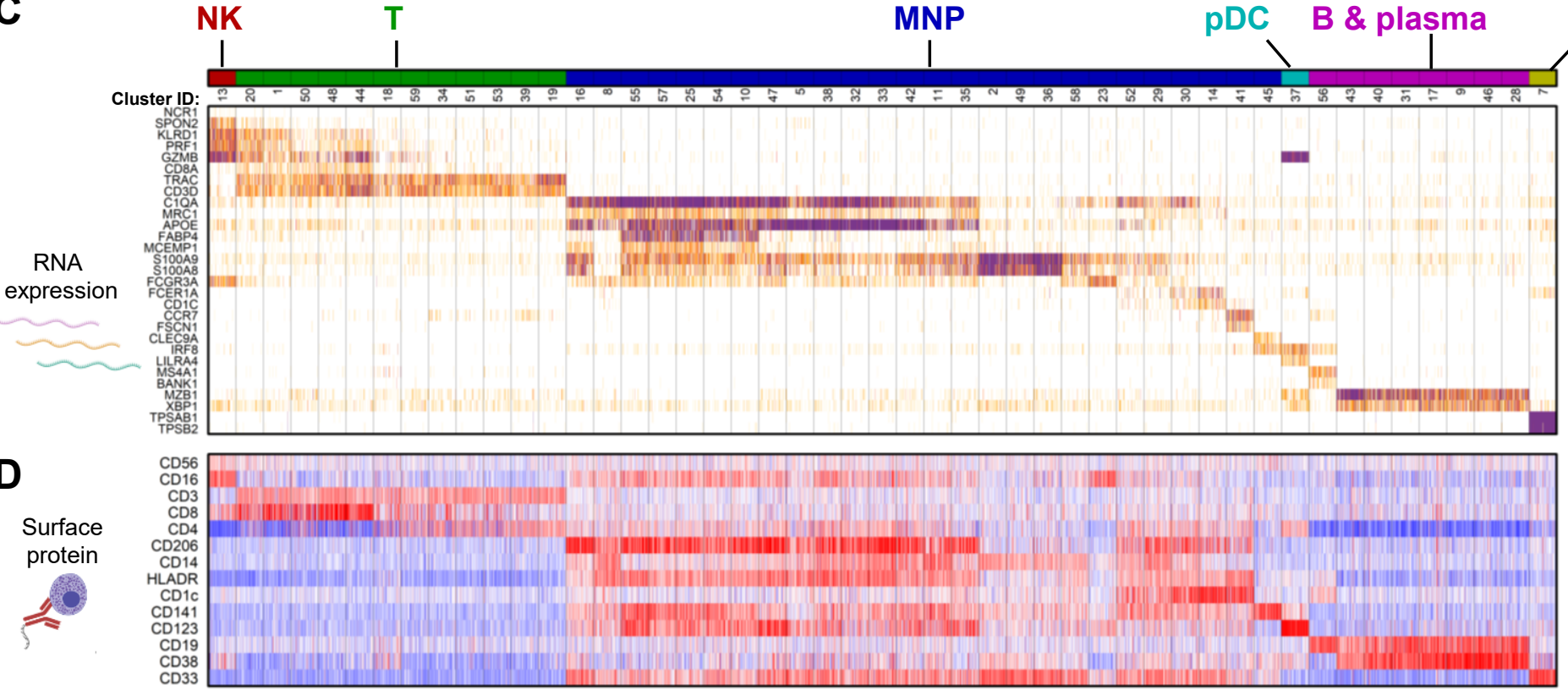

- Female

- Male

- LUAD

$\triangle$ LUSC

E

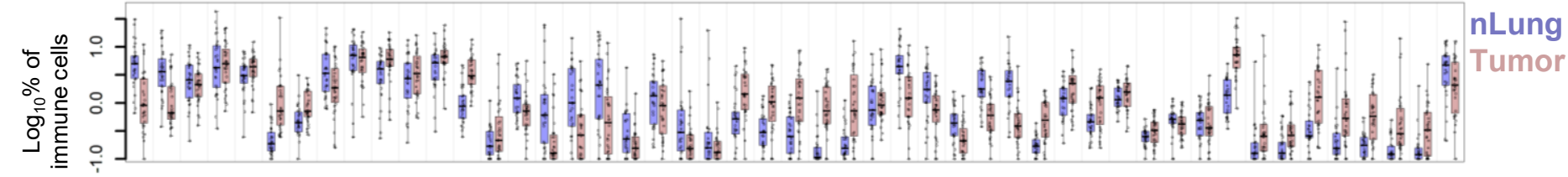

$\mathbf{F}$

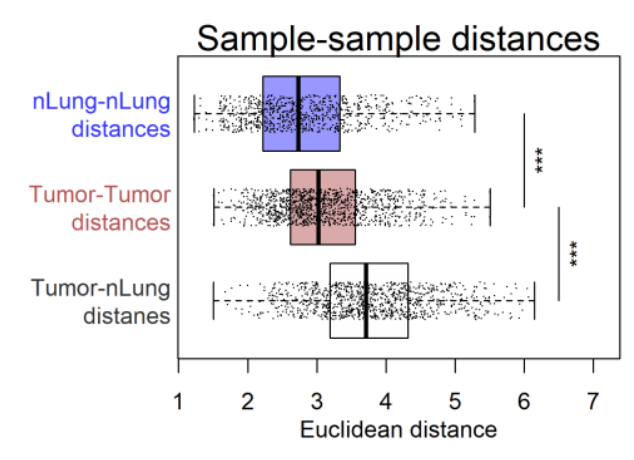

G

Log2FC: Tumor vs. nLung

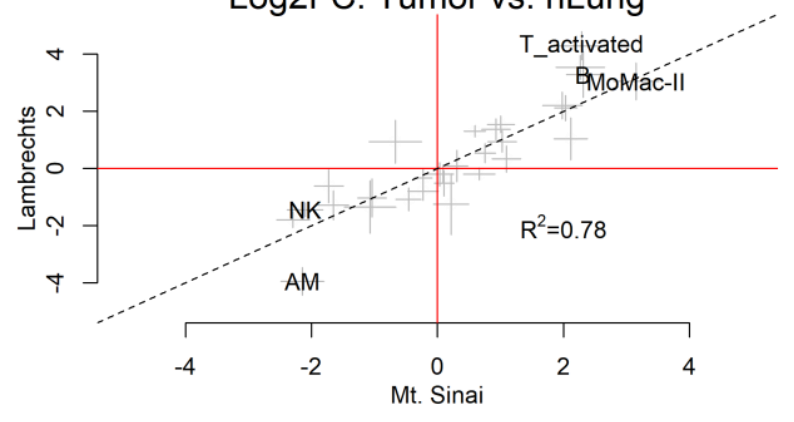

Log2(counts / row median)

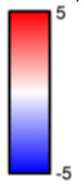




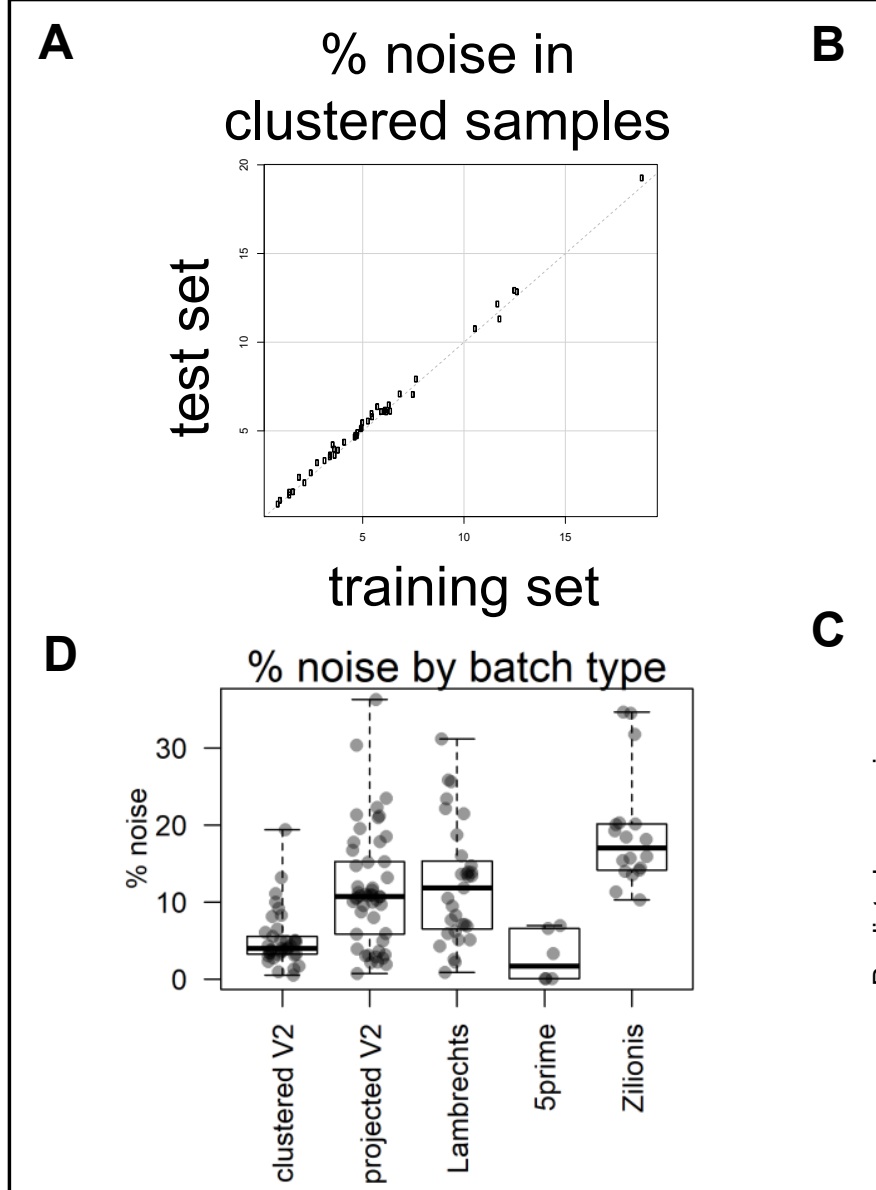

E

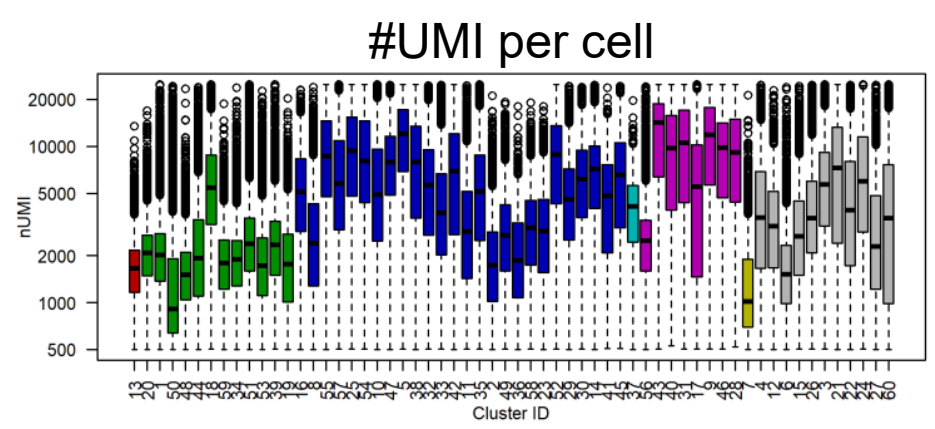

G

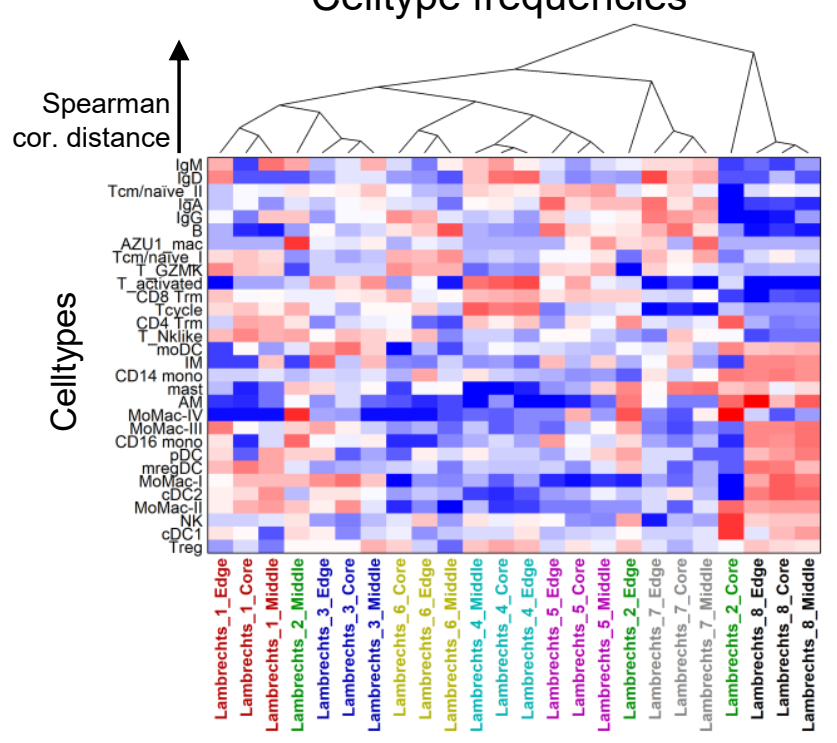

Sample

\section{Noise correction example: Treg model vs. Treg observed}

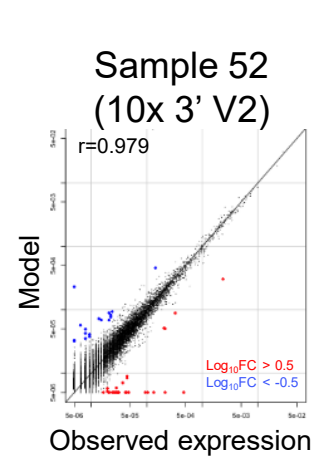

Sample 52

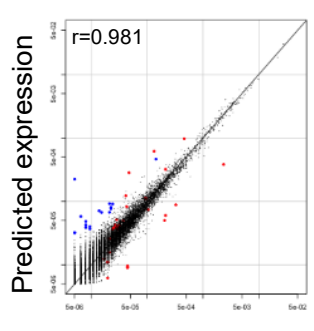

Observed expression

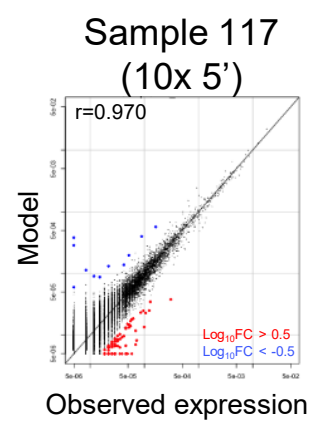

Sample 117

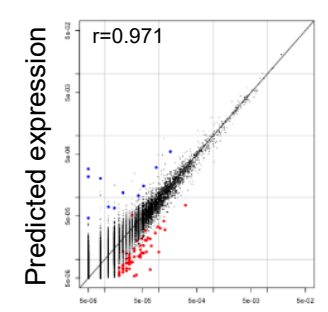

Observed expression
Sample 380

( 3 ' indrop from

Zilionis et al)

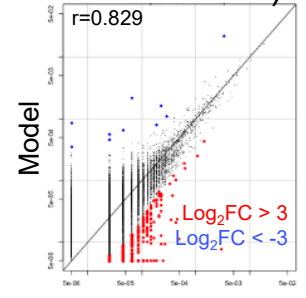

Observed expression

Sample 380

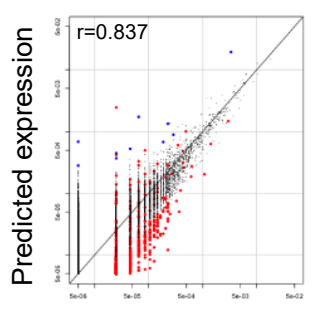

Observed expression

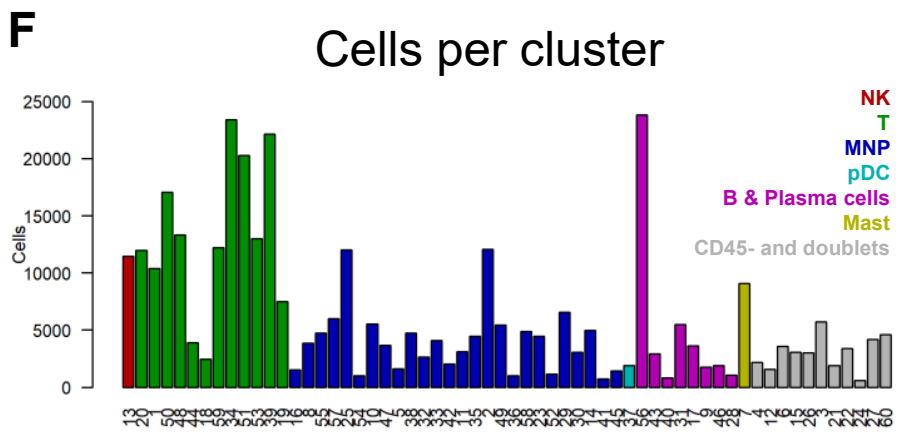

H

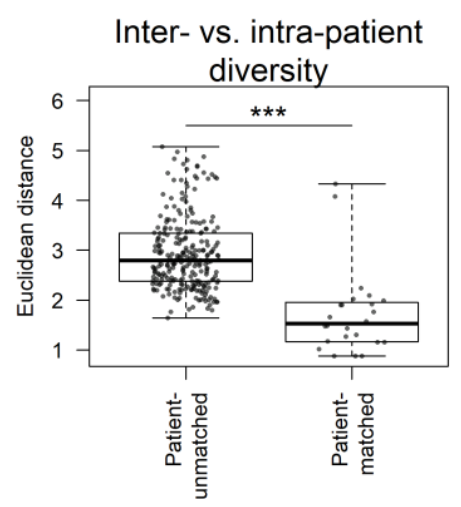


bioRxiv preprint doi: https://doi.org/10.1101/2020.07.16.207605; this version posted July 22, 2020. The copyright holder for this preprint (which
Fiaure ?

Figure 2 was not certified by peer review) is the author/funder. All rights reserved. No reuse allowed without permission.

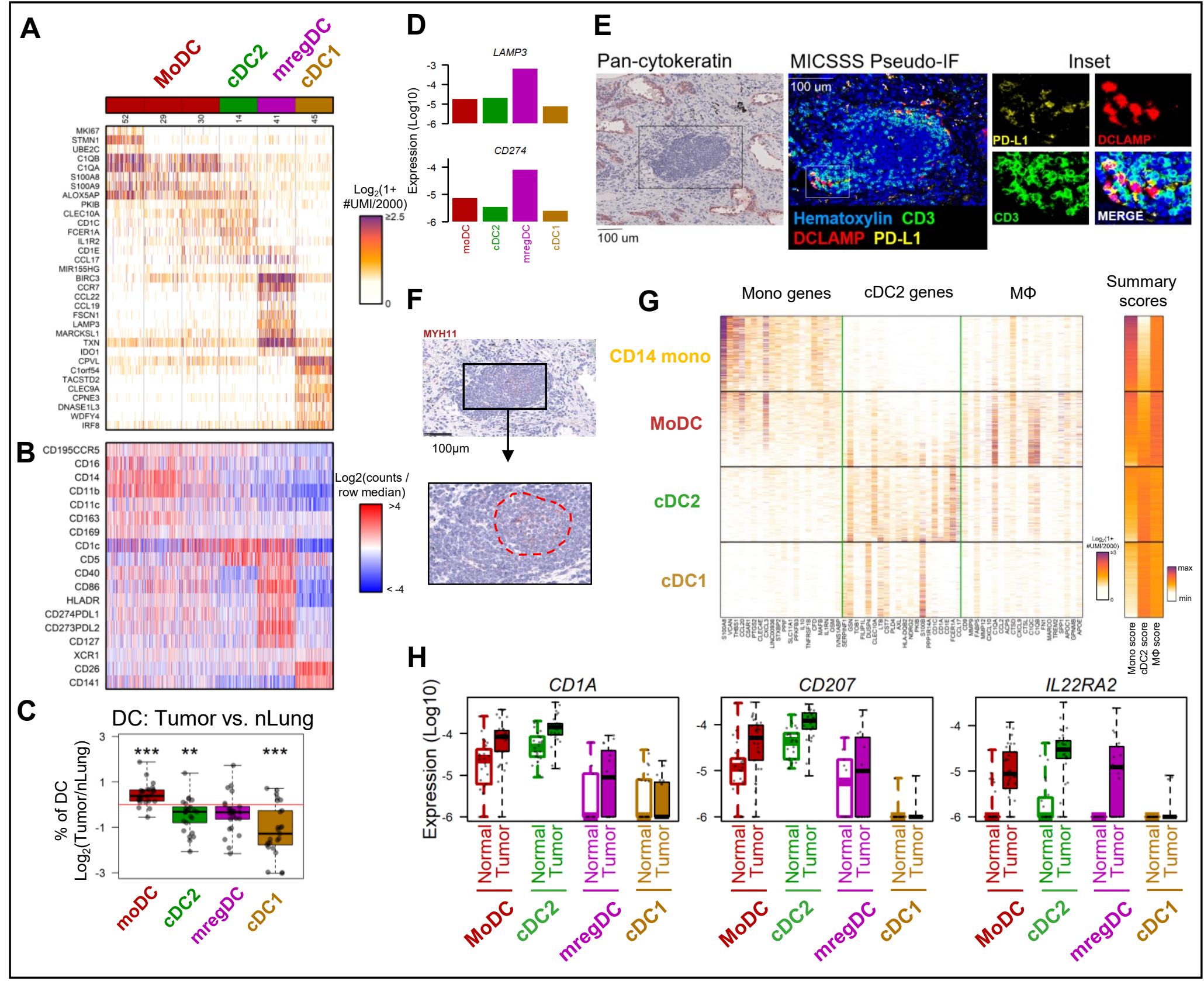


Eja broRyy preprint doi: https://doi.org/10.1101/2020.07.16.207605; this version posted July 22, 2020. The copyright holder for this preprint (which Figure S2 was not certified by peer review) is the author/funder. All rights reserved. No reuse allowed without permission.

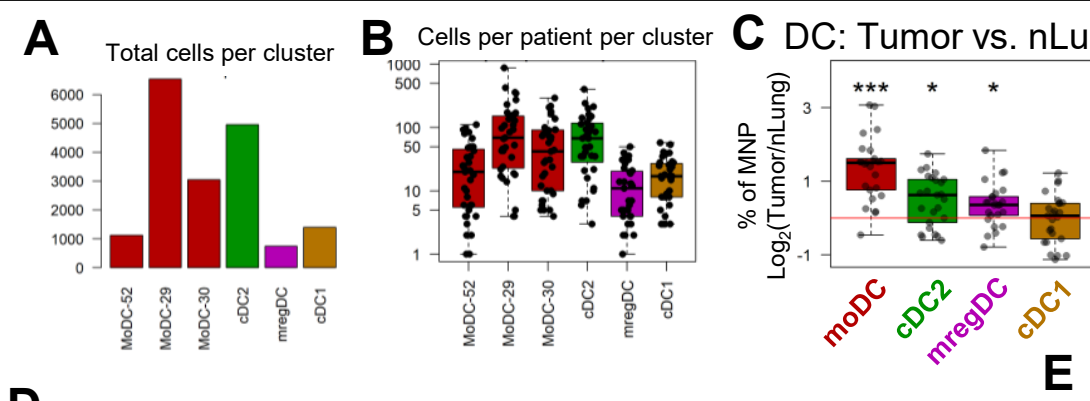

D

A Total cells per cluster $\mathbf{B}$ Cells per patient per cluster $\mathbf{C}$ DC: Tumor vs. nLung
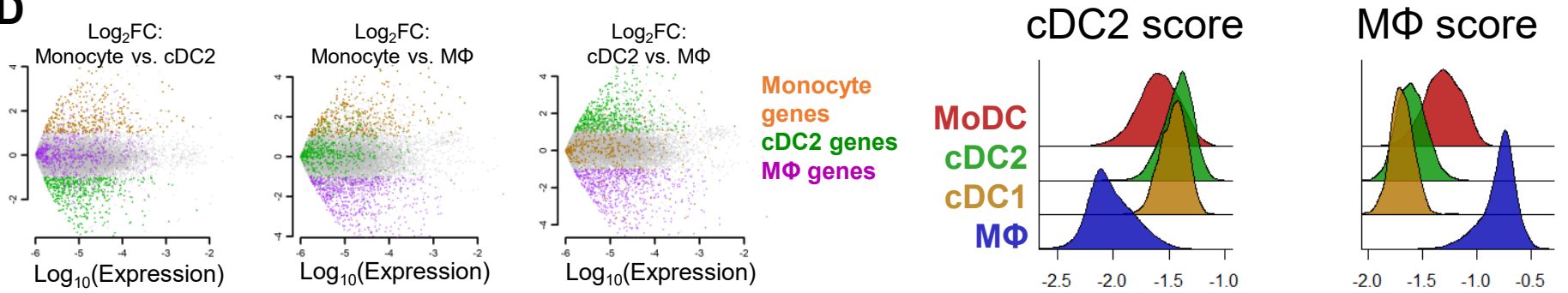

$\mathbf{F}$

Gene module correlation in DCs

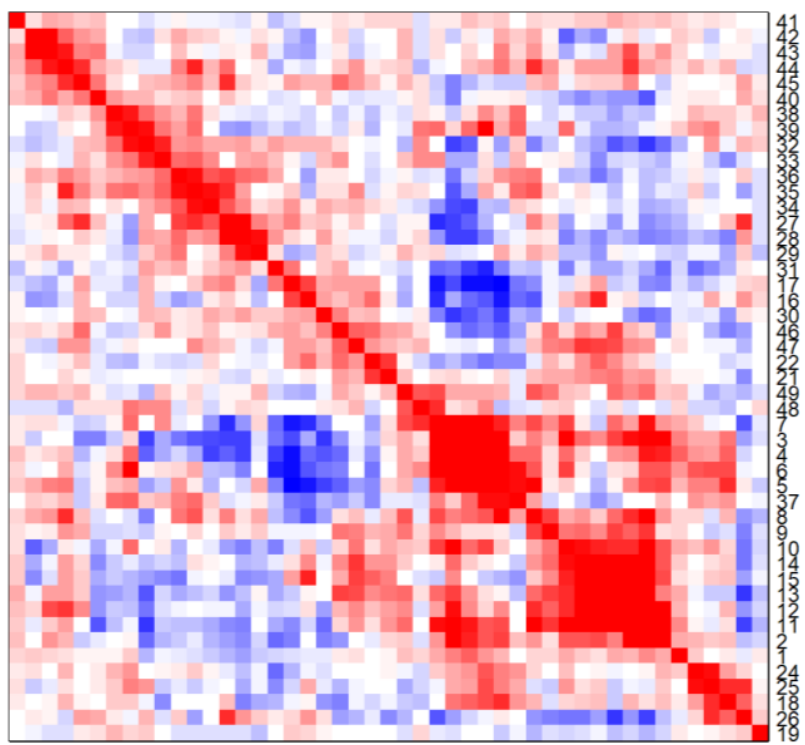

G Most correlated genes

H within each module

Inter-module cor

$<-0.35>0.35$
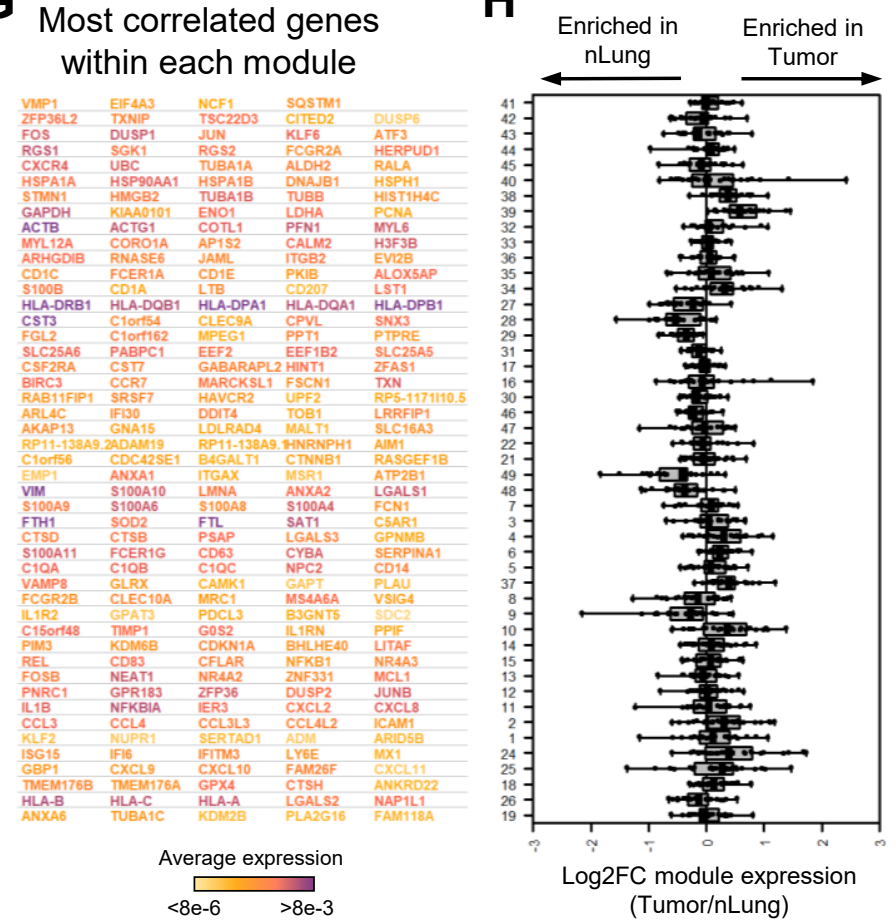

Average module expression
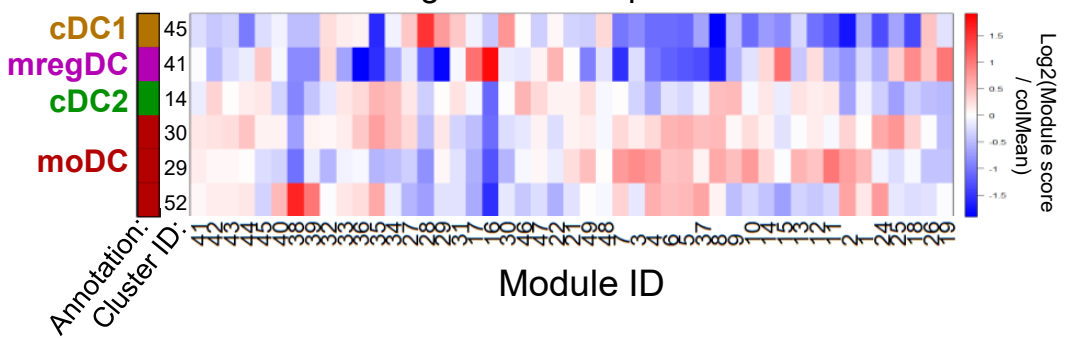

DC from Lambrechts, et al.
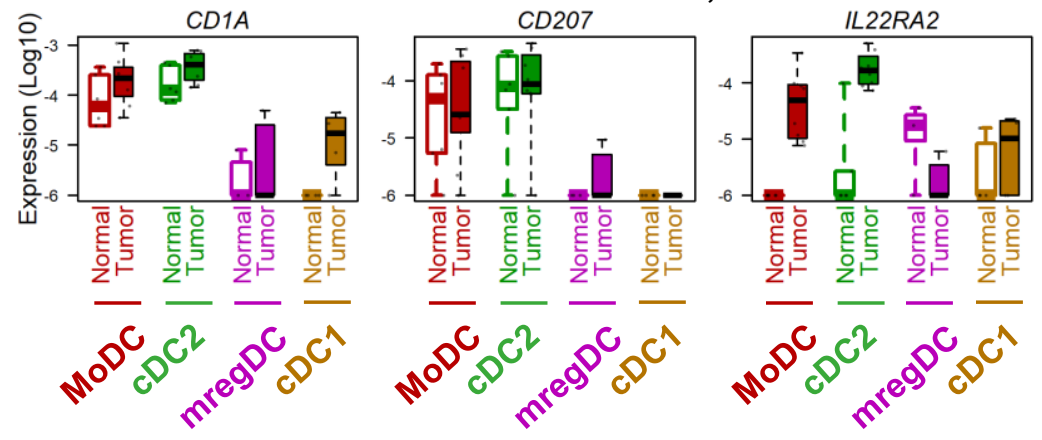


\section{A Cluster averages}

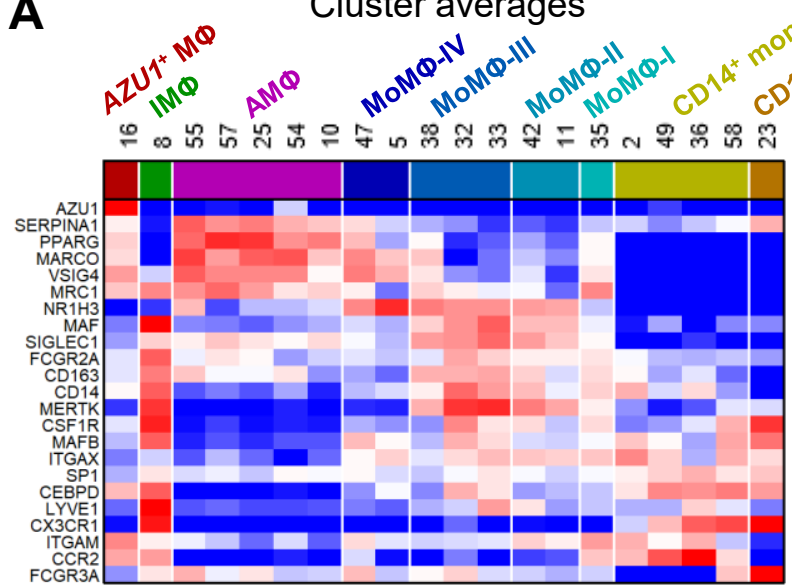

\section{B}

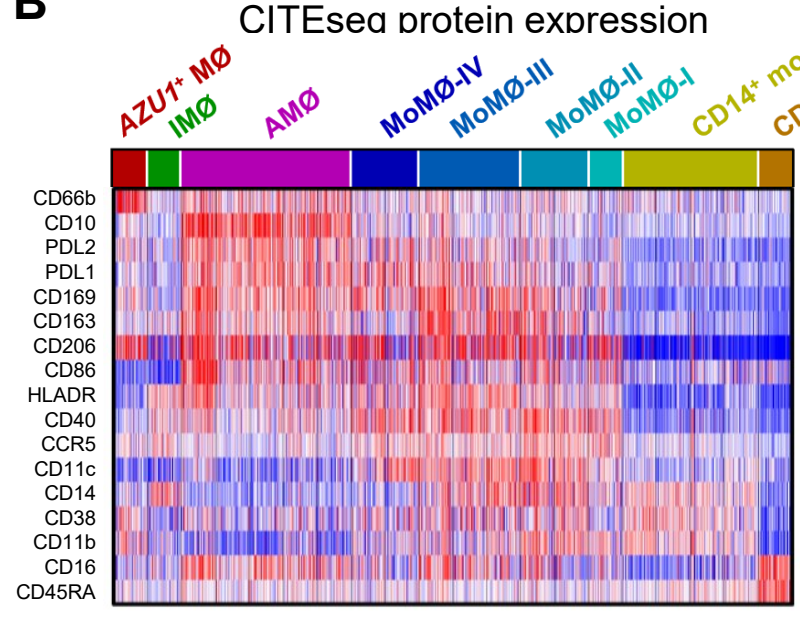

G

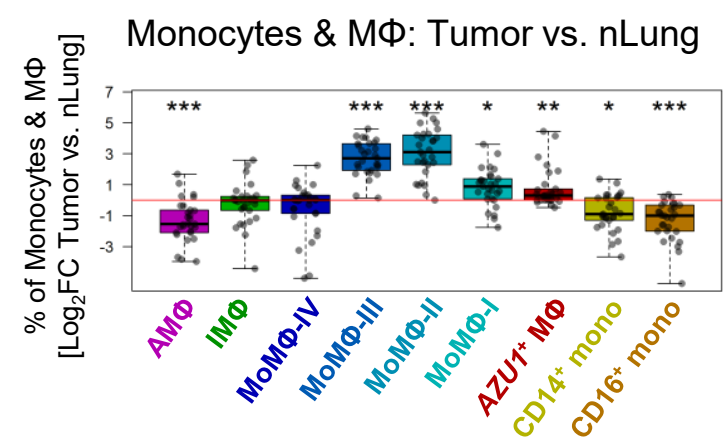

H

\section{Secreted factors}

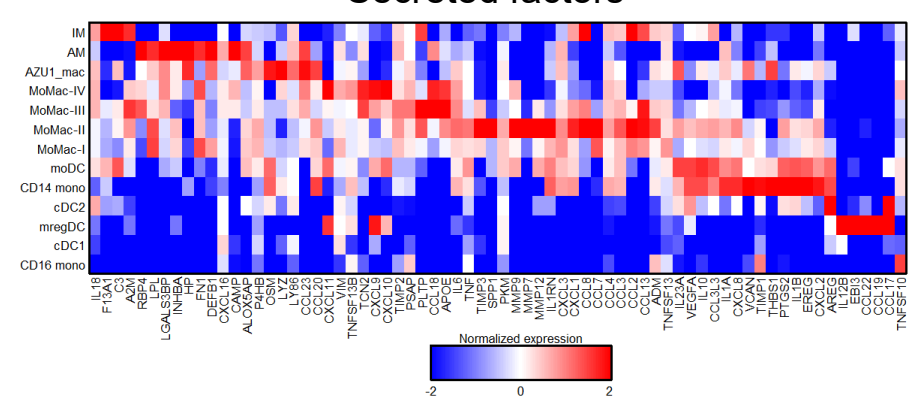

C

Module expression scores Mod. ID: 29: C1Q genes
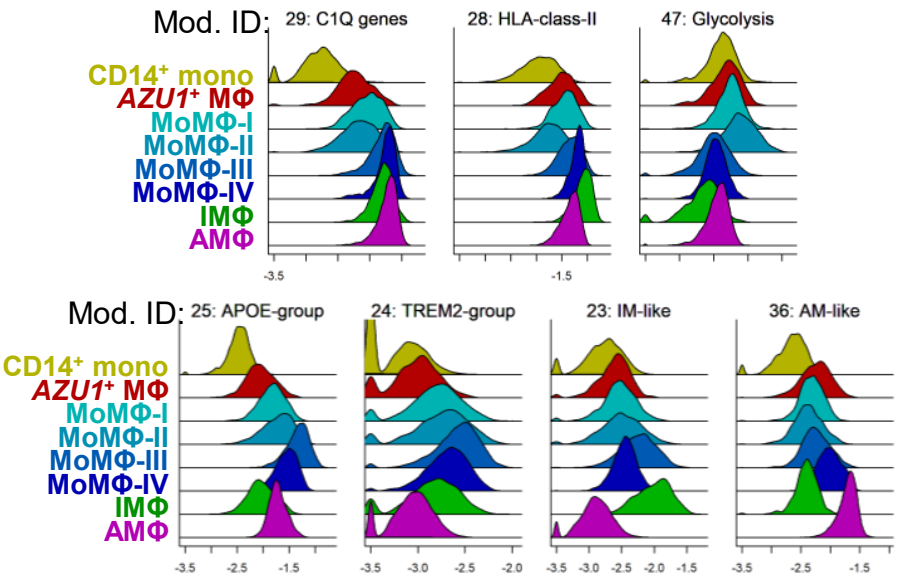

D

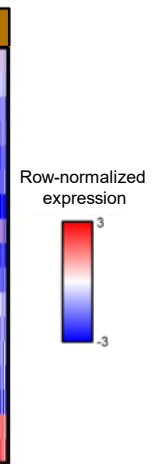

$\mathbf{F}$

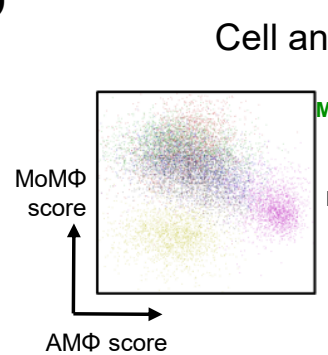

E CD14+ monocyte score

F
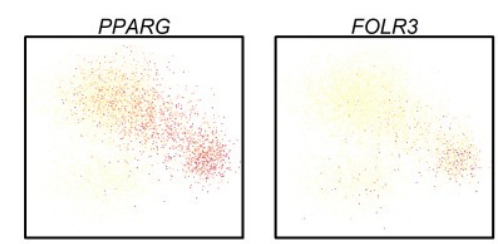

TREM1
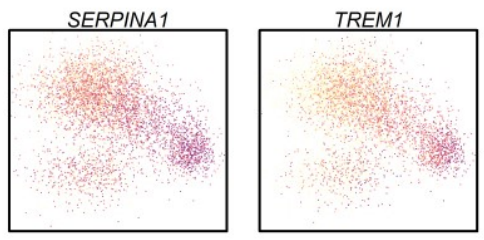

TREM2
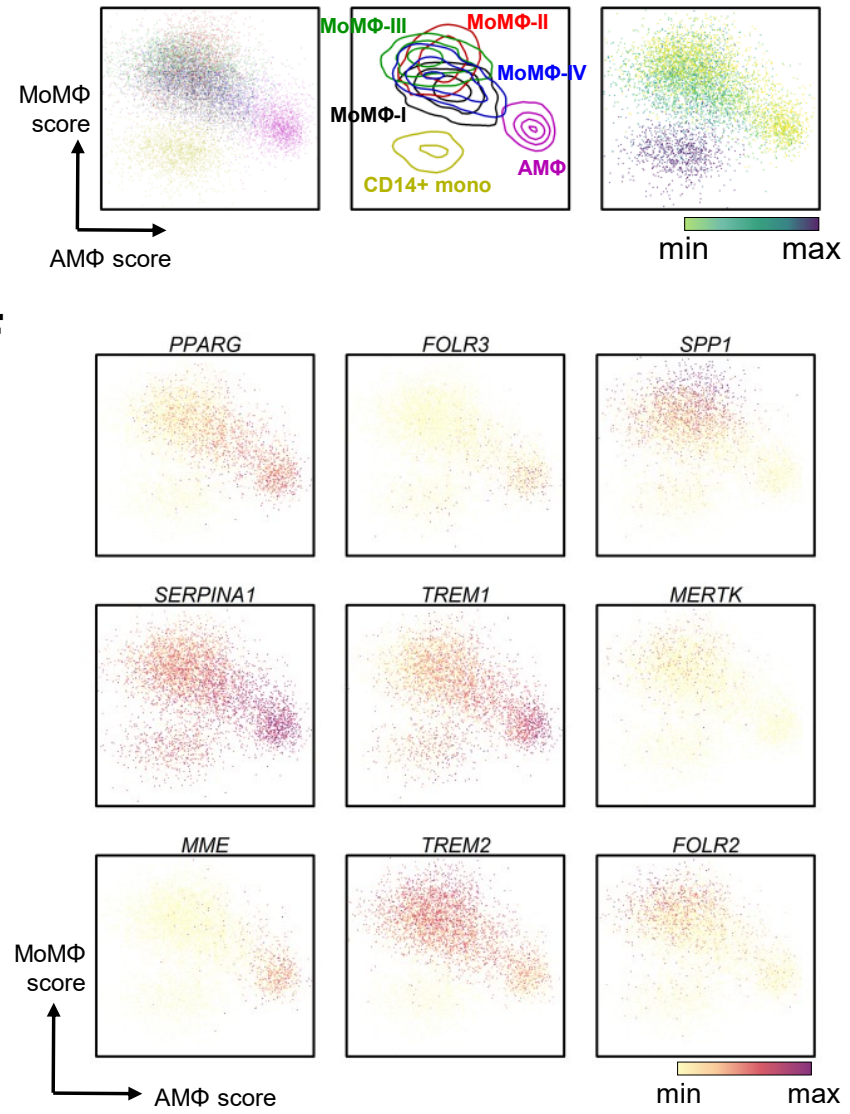

FOLR2

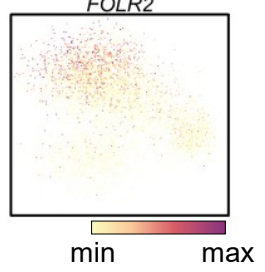




\section{A}

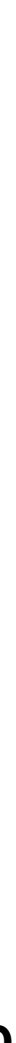

D

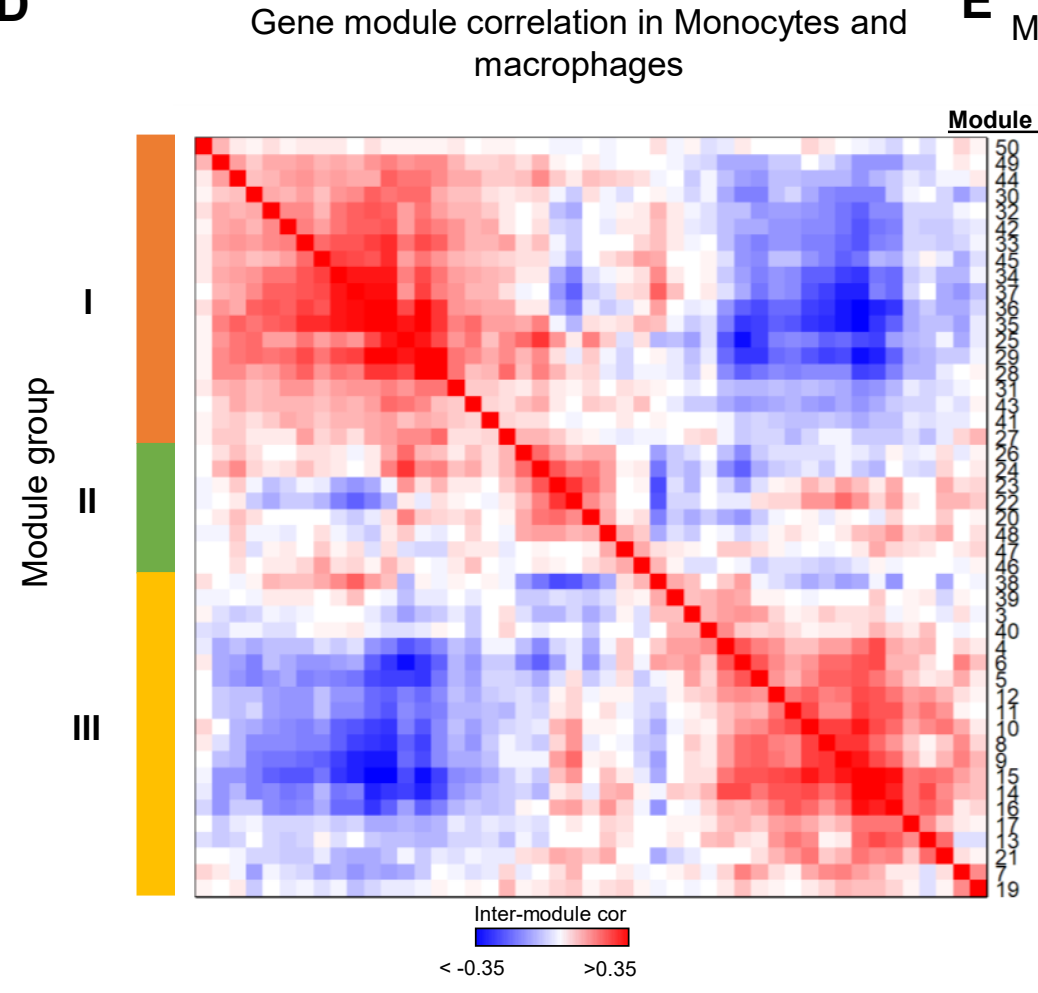

G

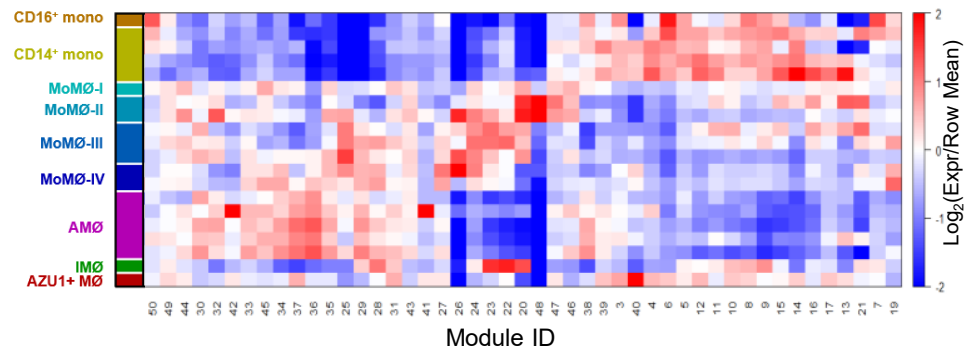

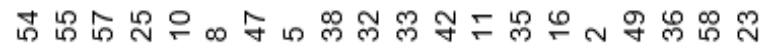

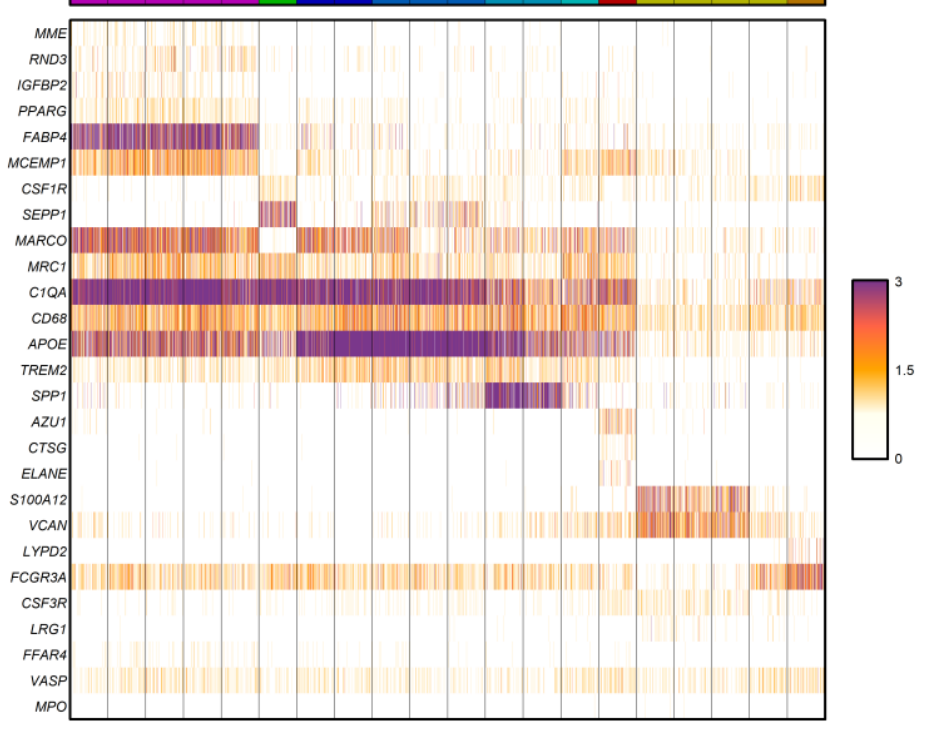

Single-cell expression

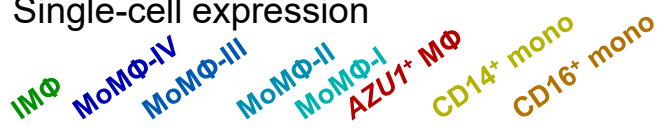

B
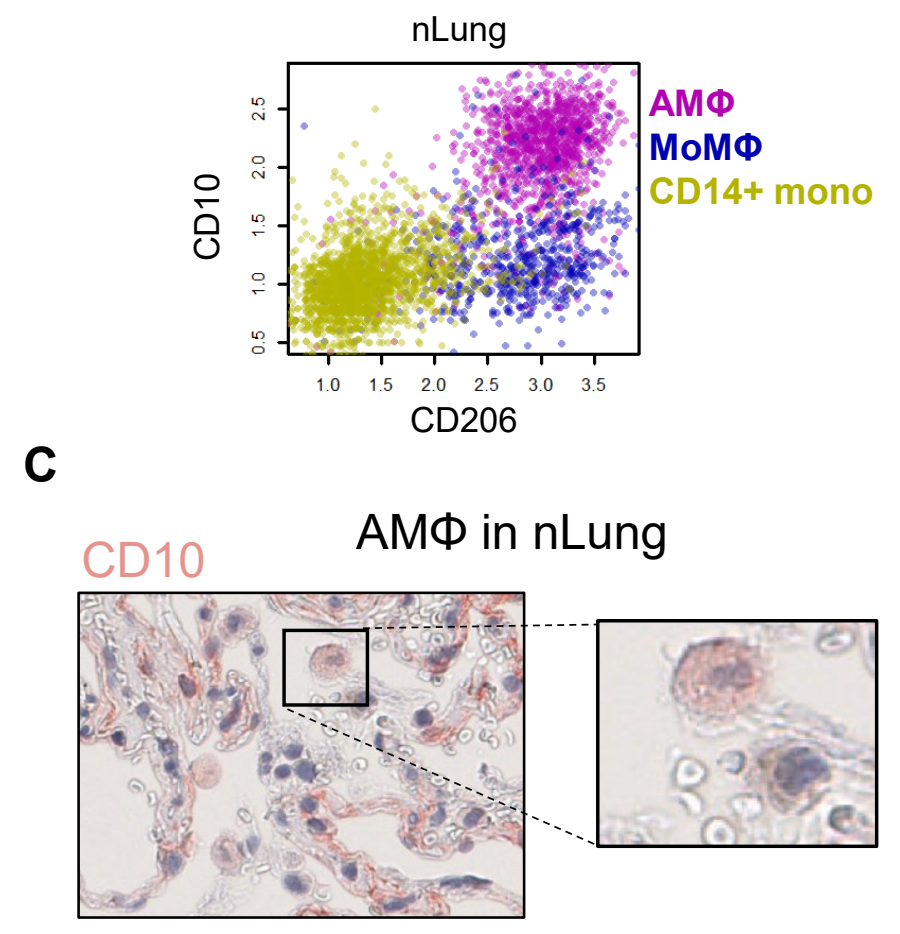

C

\section{E Most correlated genes within \\ F} each module

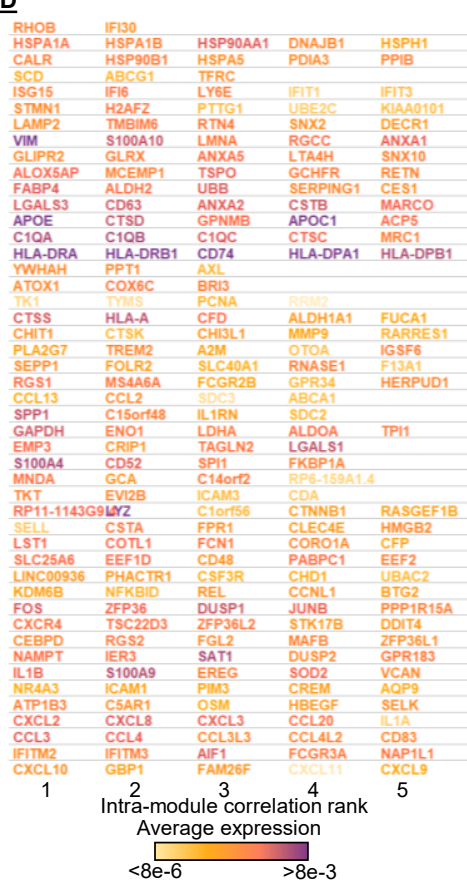

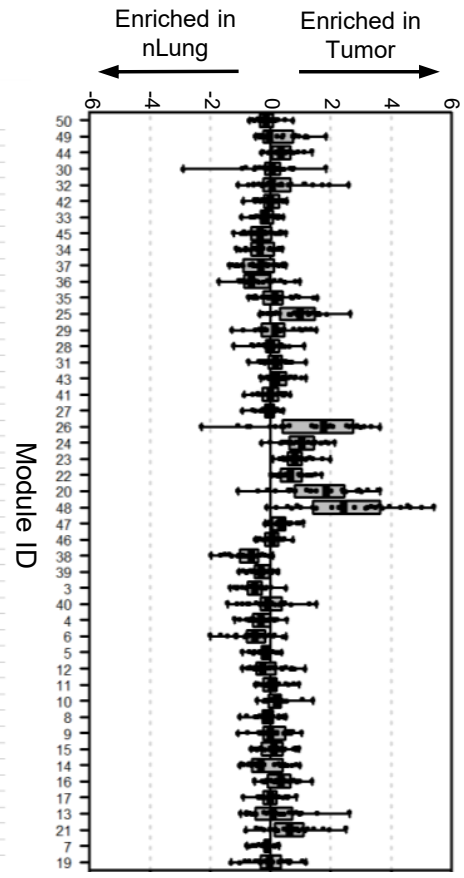

Log2FC module expression (Tumor/nLung)

H

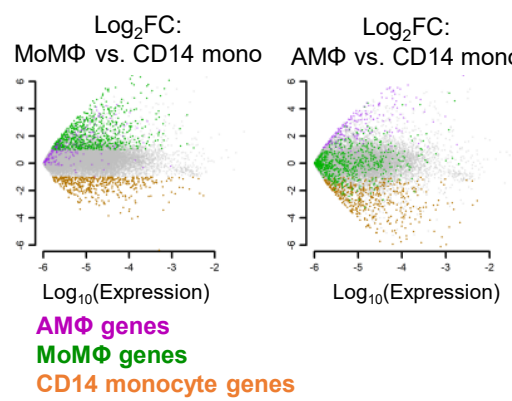

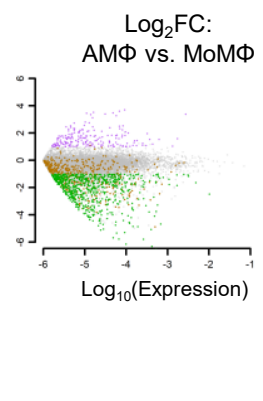


Figefreor4rint doi: https://doi.org/10.1101/2020.07.16.207605; this version posted July 22, 2020. The copyright holder for this preprint (which was not certified by peer review) is the author/funder. All rights reserved. No reuse allowed without permission.

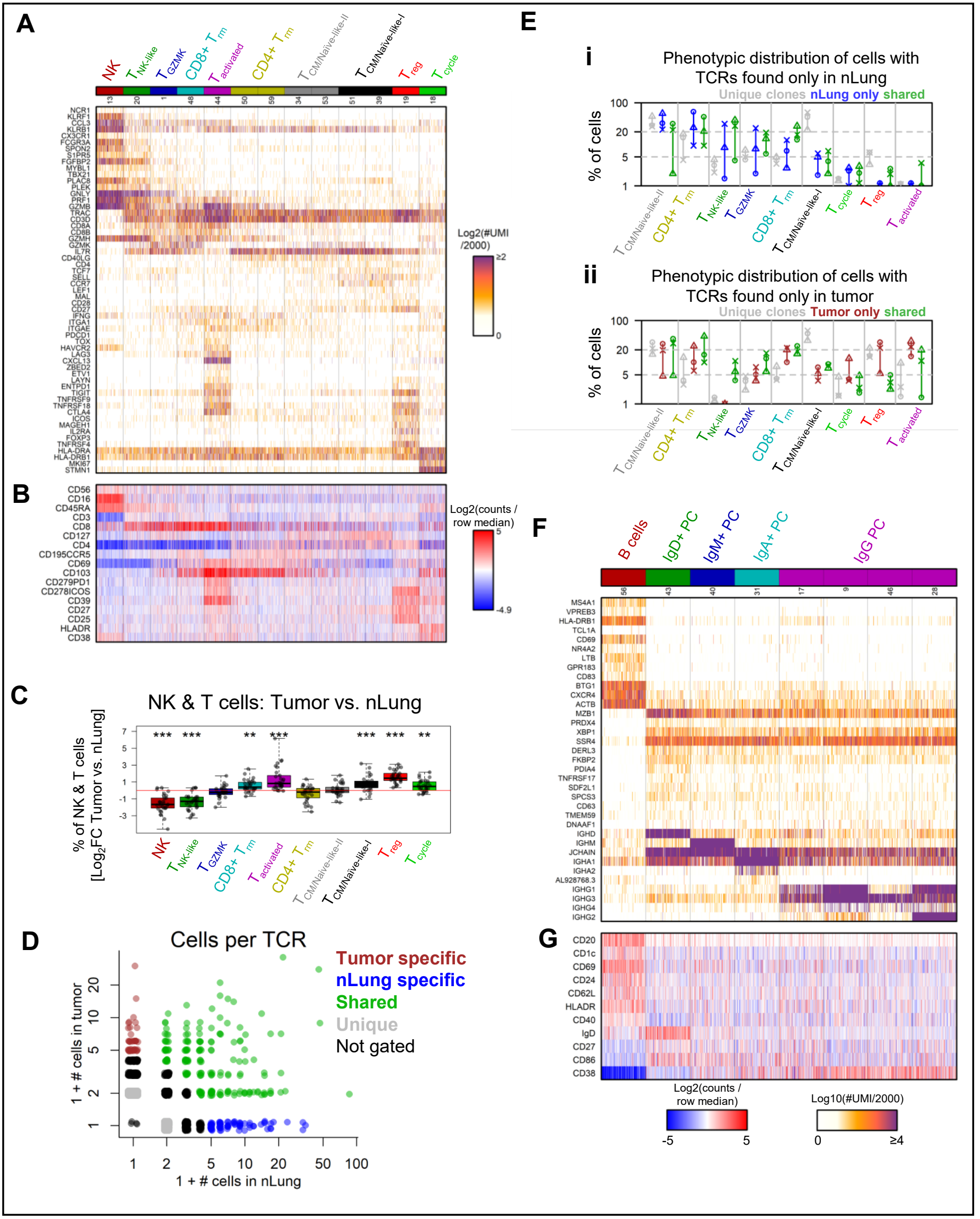




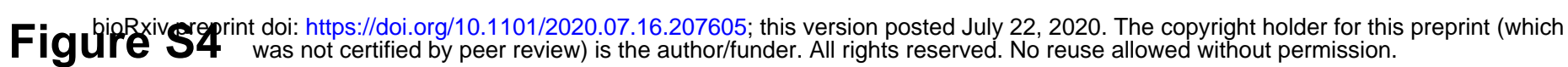

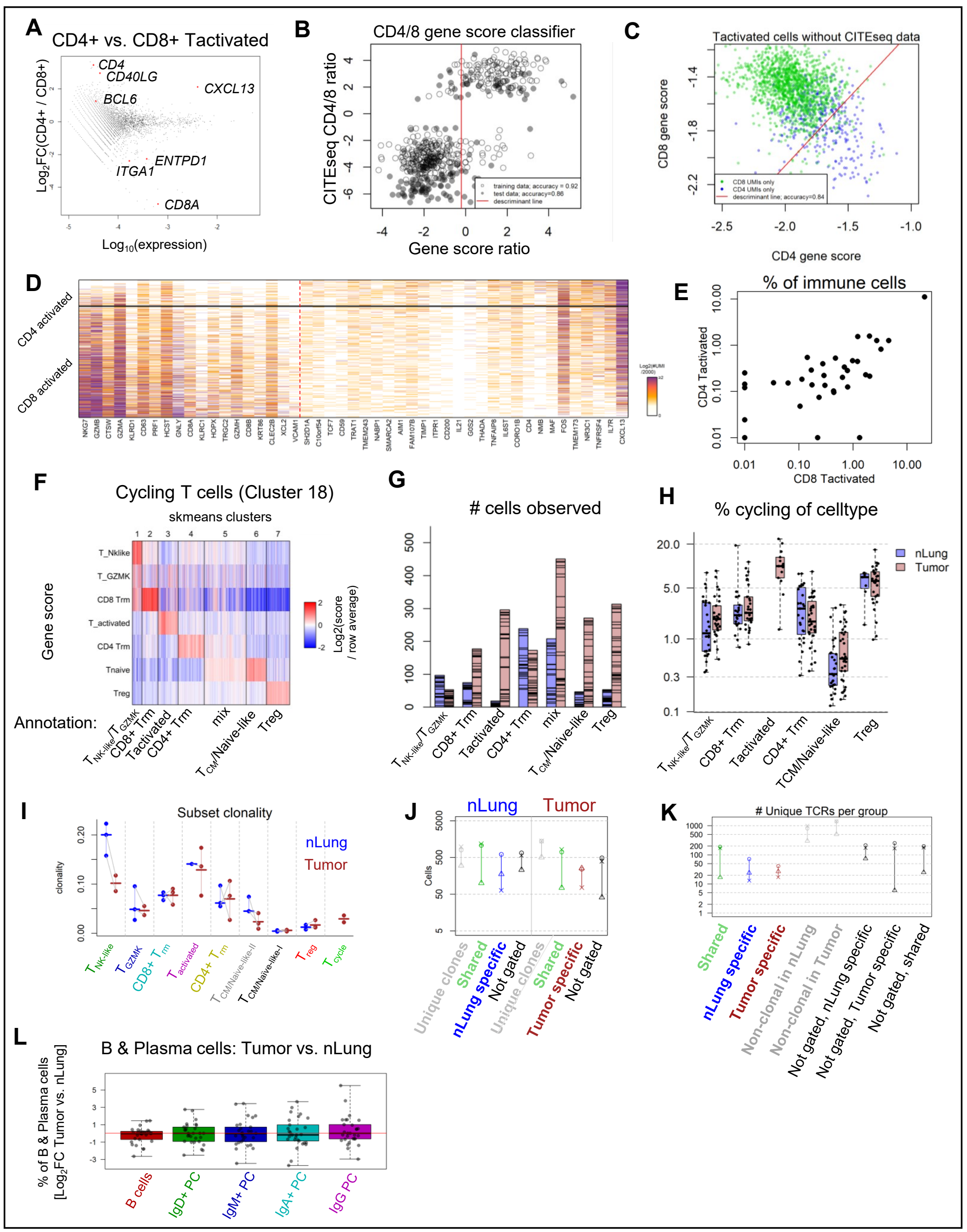


bioRxiv preprint doi: https://doi.org/10.1101/2020.07.16.207605; this version posted July 22, 2020. The copyright holder for this preprint (which

Figure 5

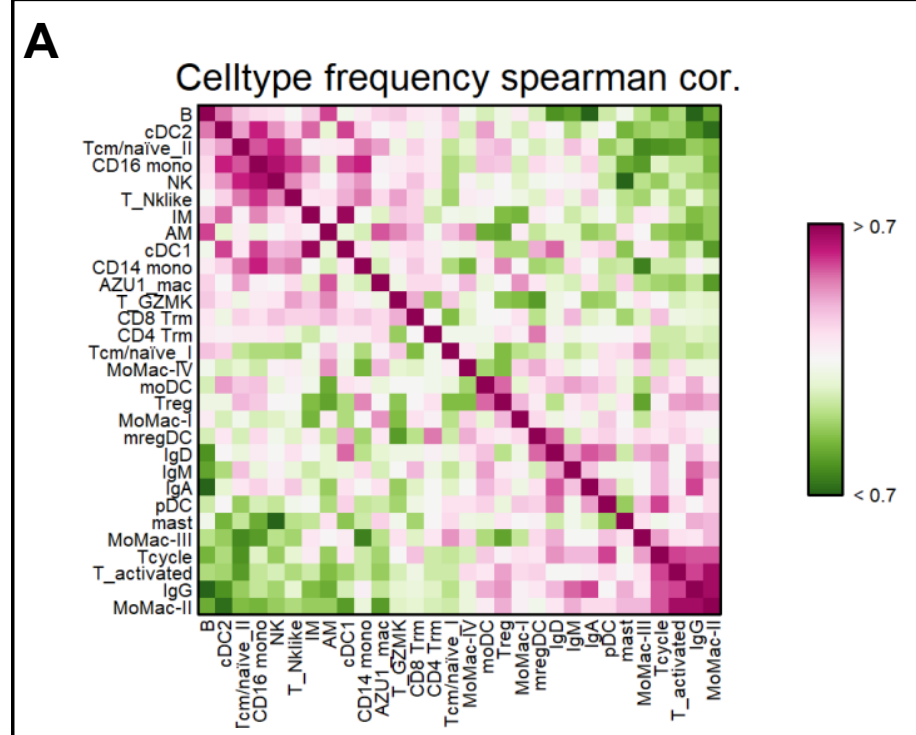

D

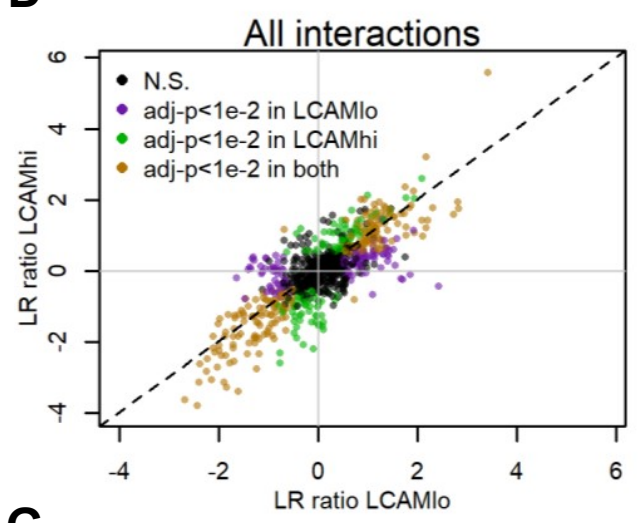

G

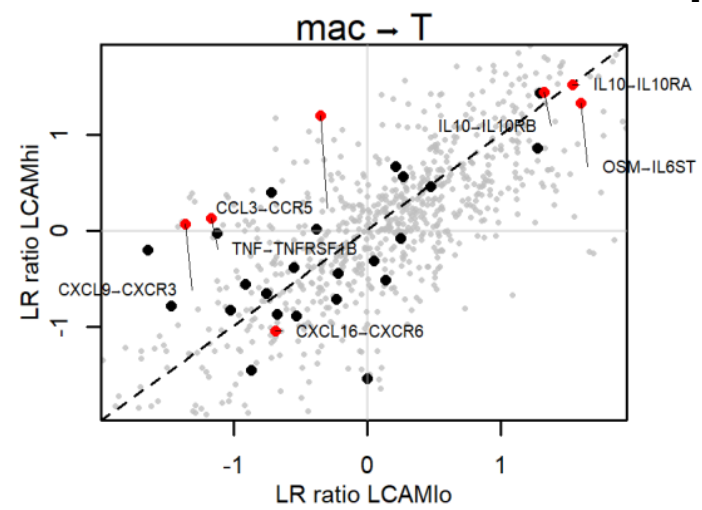

E

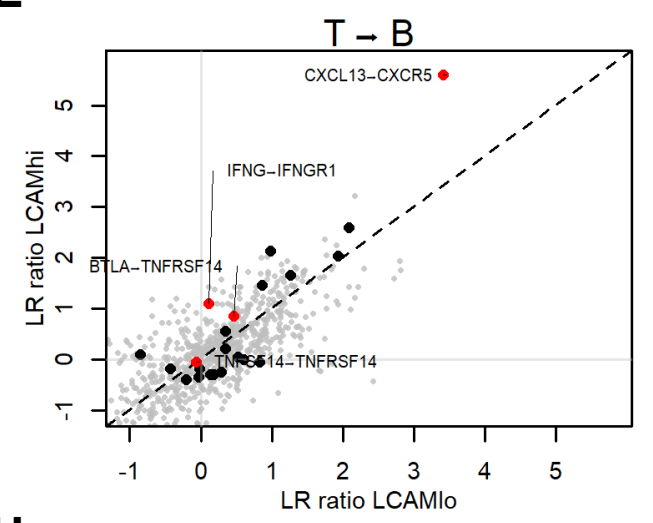

H

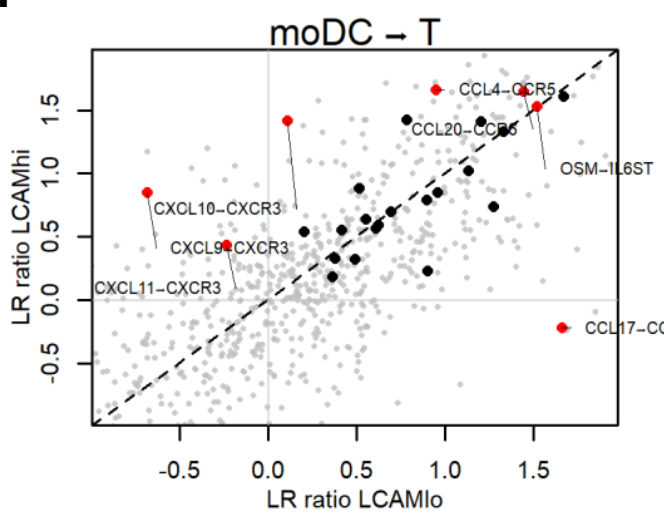

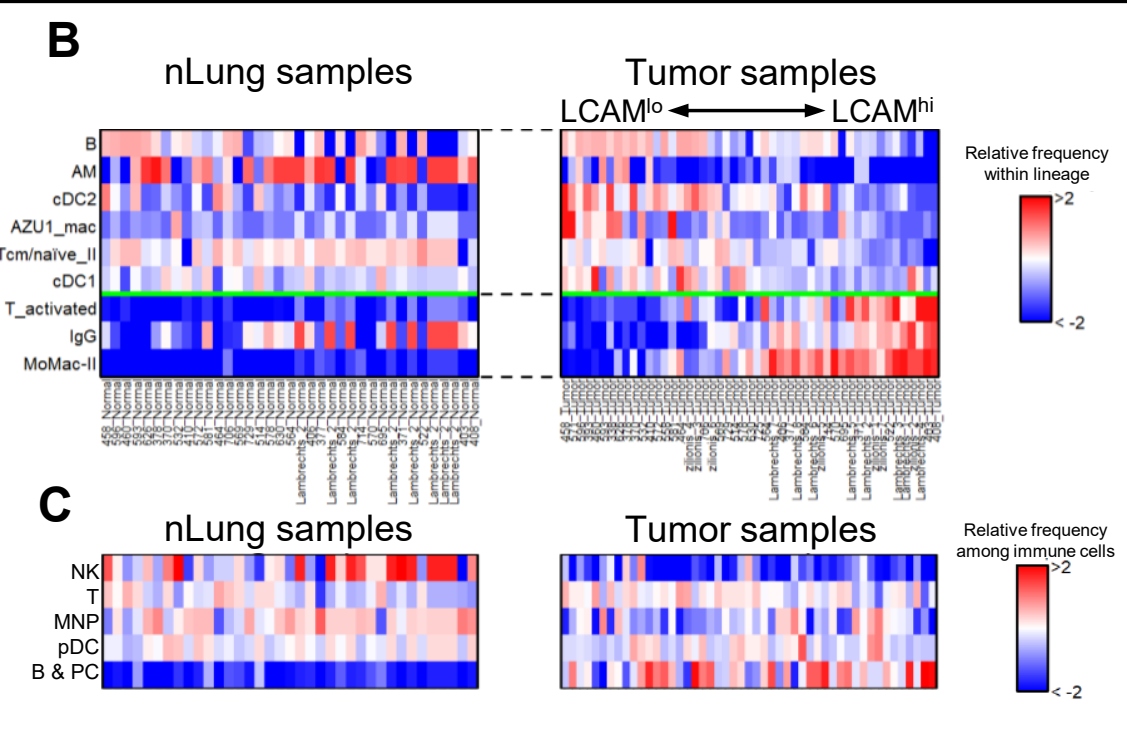

F

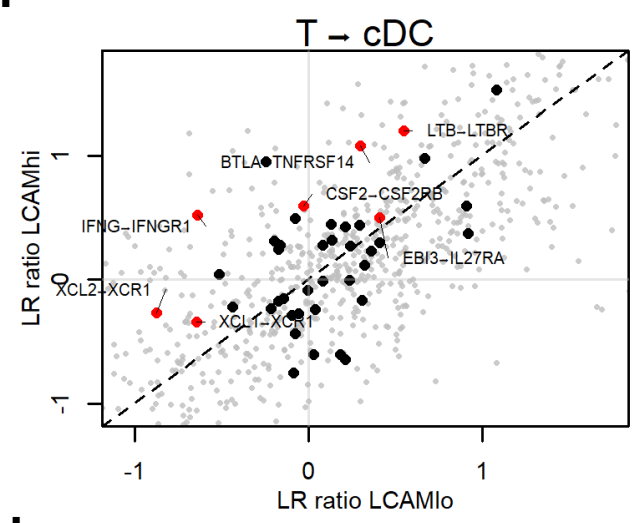

I

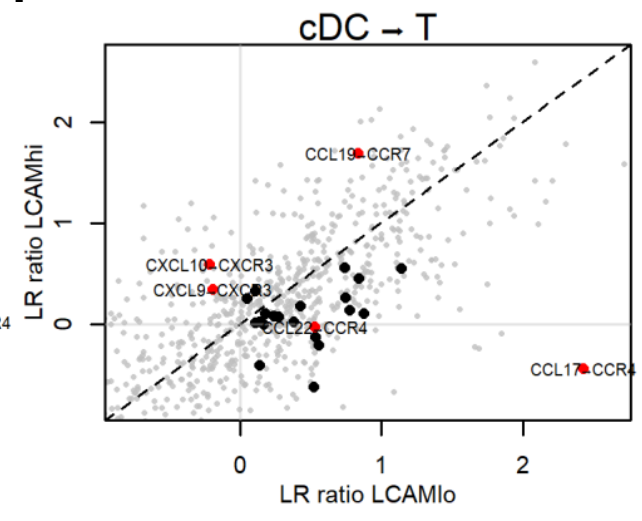


Figure S5

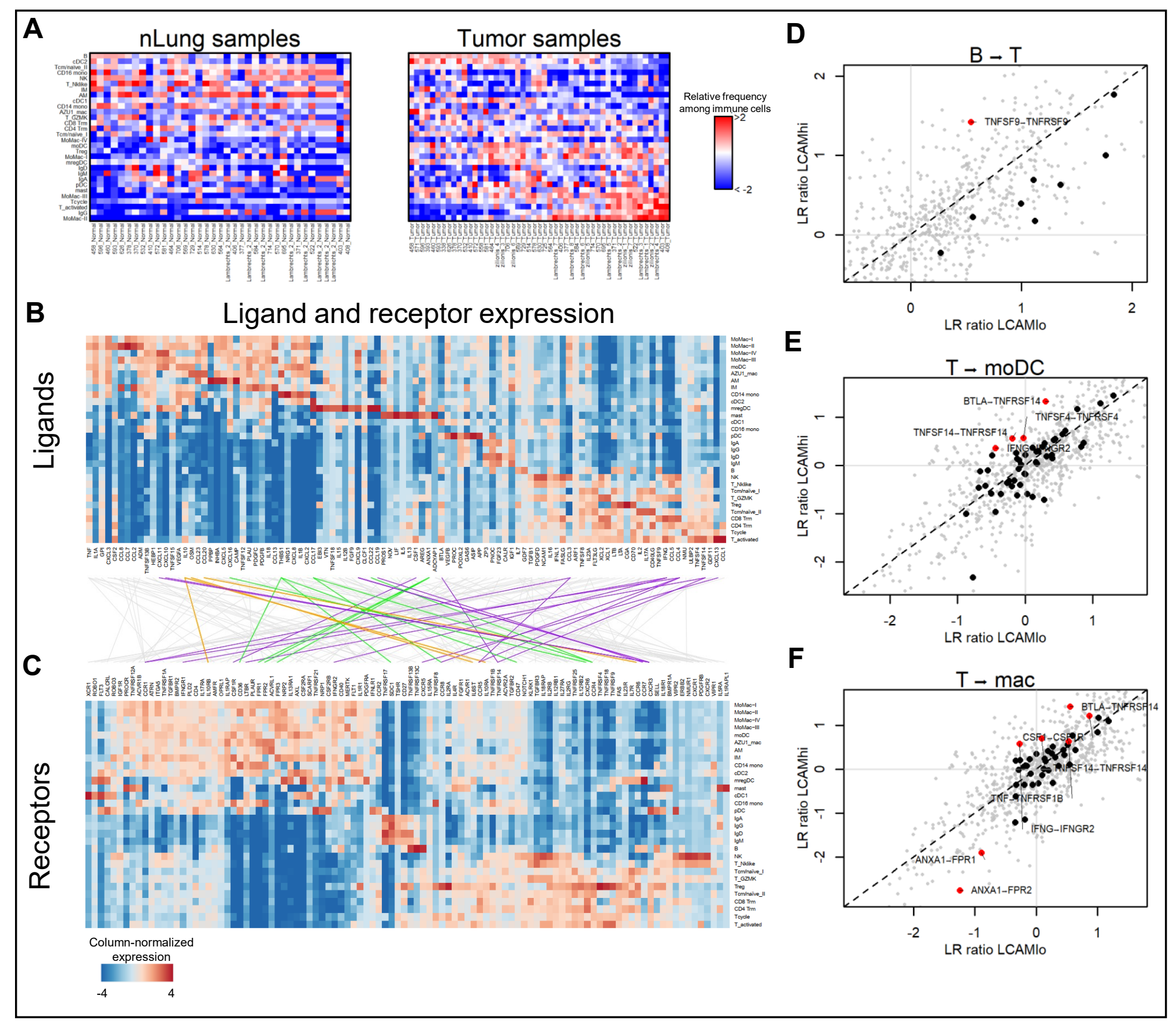


Figure 6

A

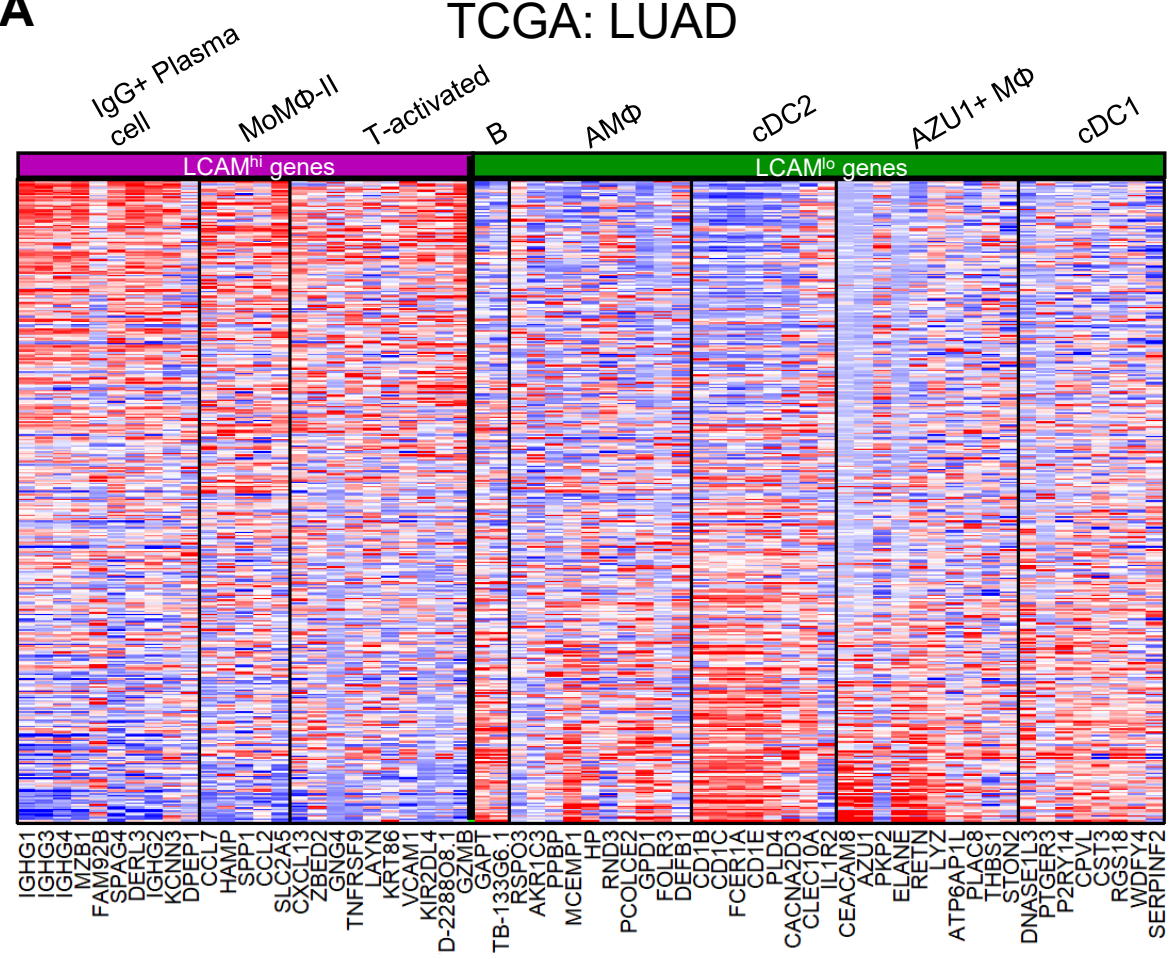

E Smoking signature present
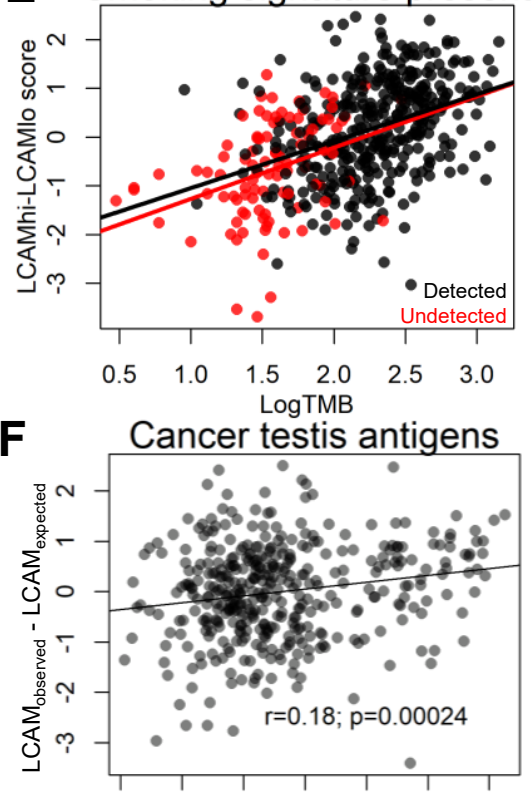

$\begin{array}{lllllll}0.3 & 0.4 & 0.5 & 0.6 & 0.7 & 0.8 & 0.9\end{array}$ CTA score
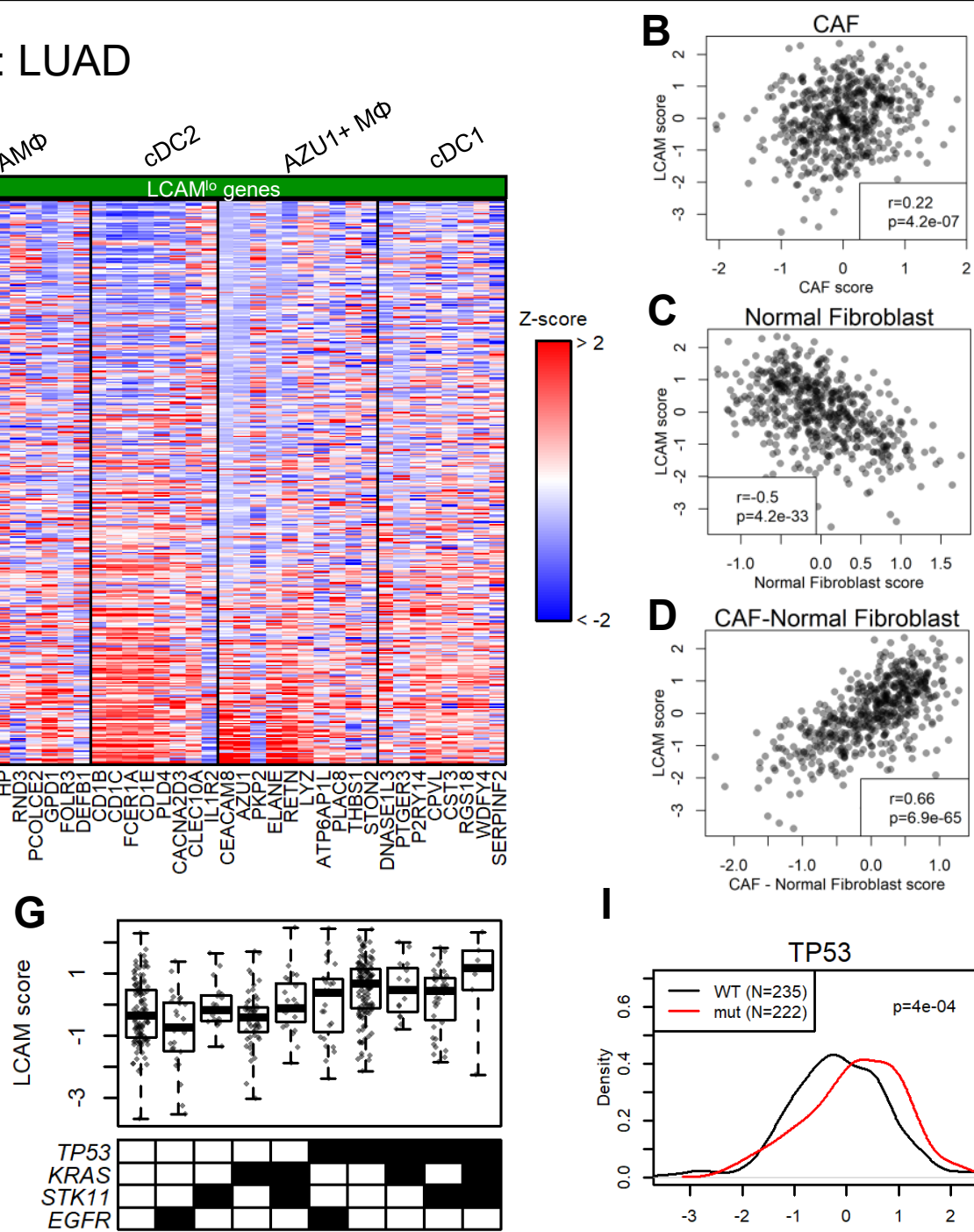

C Normal Fibroblast

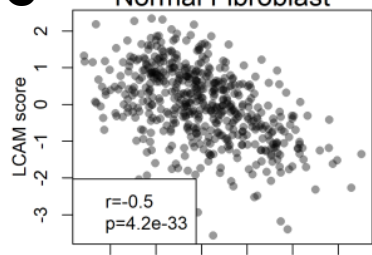

$\begin{array}{lllll}-1.0 & 0.0 & 0.5 & 1.0 & 1.5\end{array}$

D CAF-Normal Fibroblast

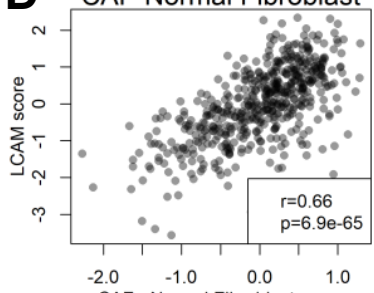

I

CAF - Normal Fibroble

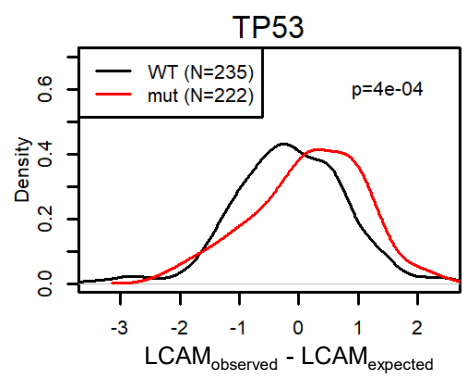

$J$

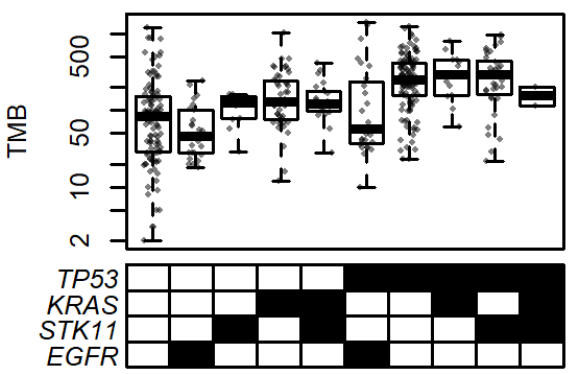

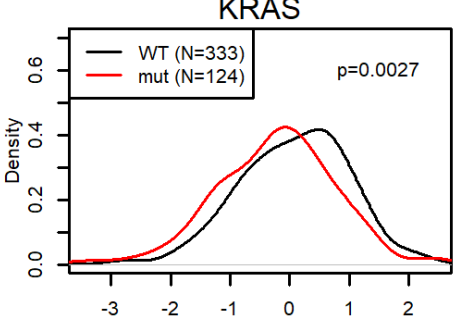

LCAM $_{\text {observed }}-$ LCAM $_{\text {expected }}$ 


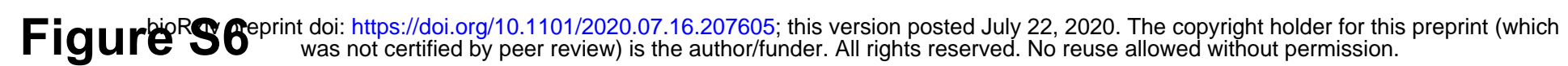

A LCAM ${ }^{\text {hi/lo }}$ DEGs B

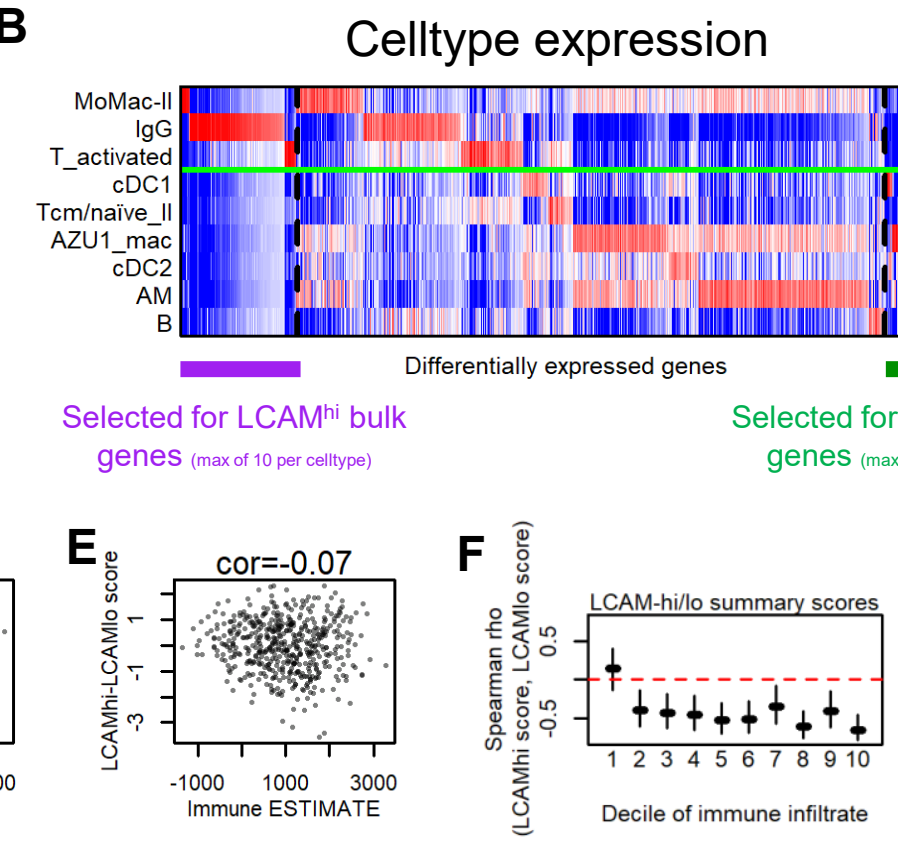

Celltype expression

Norm. expression
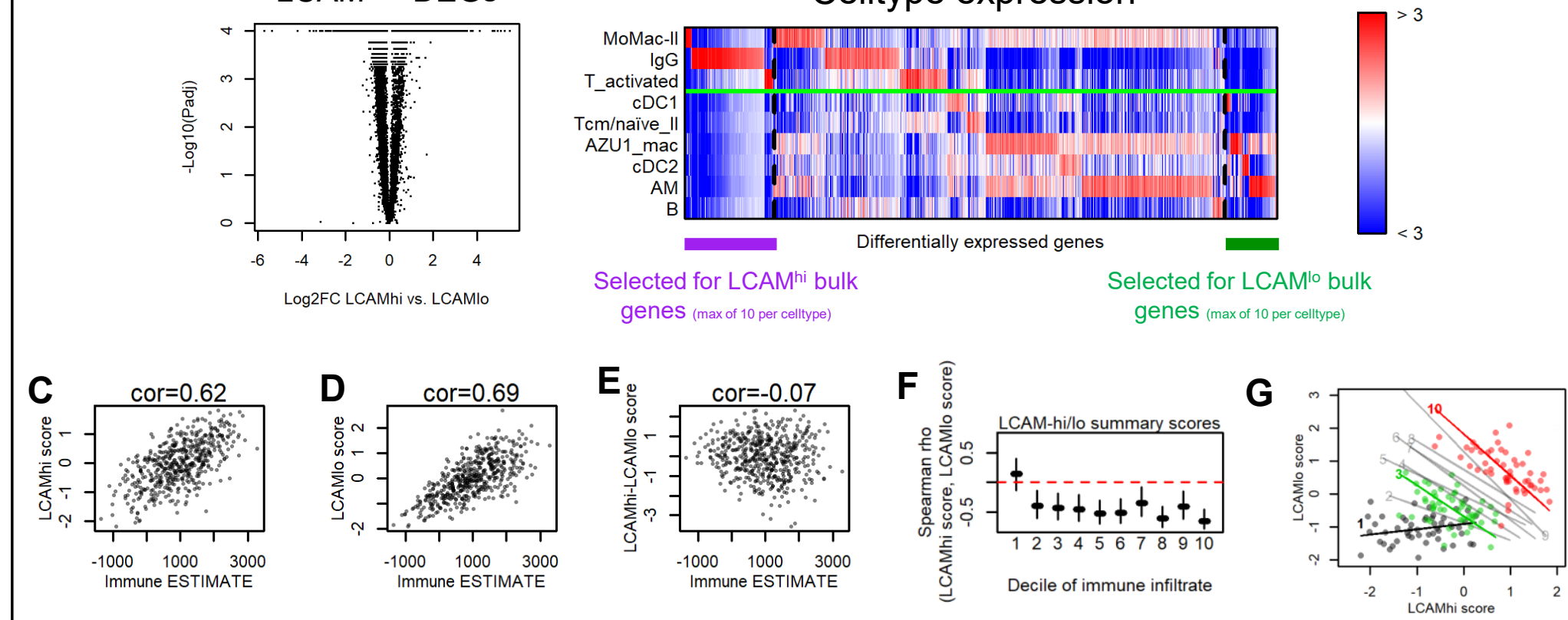

H
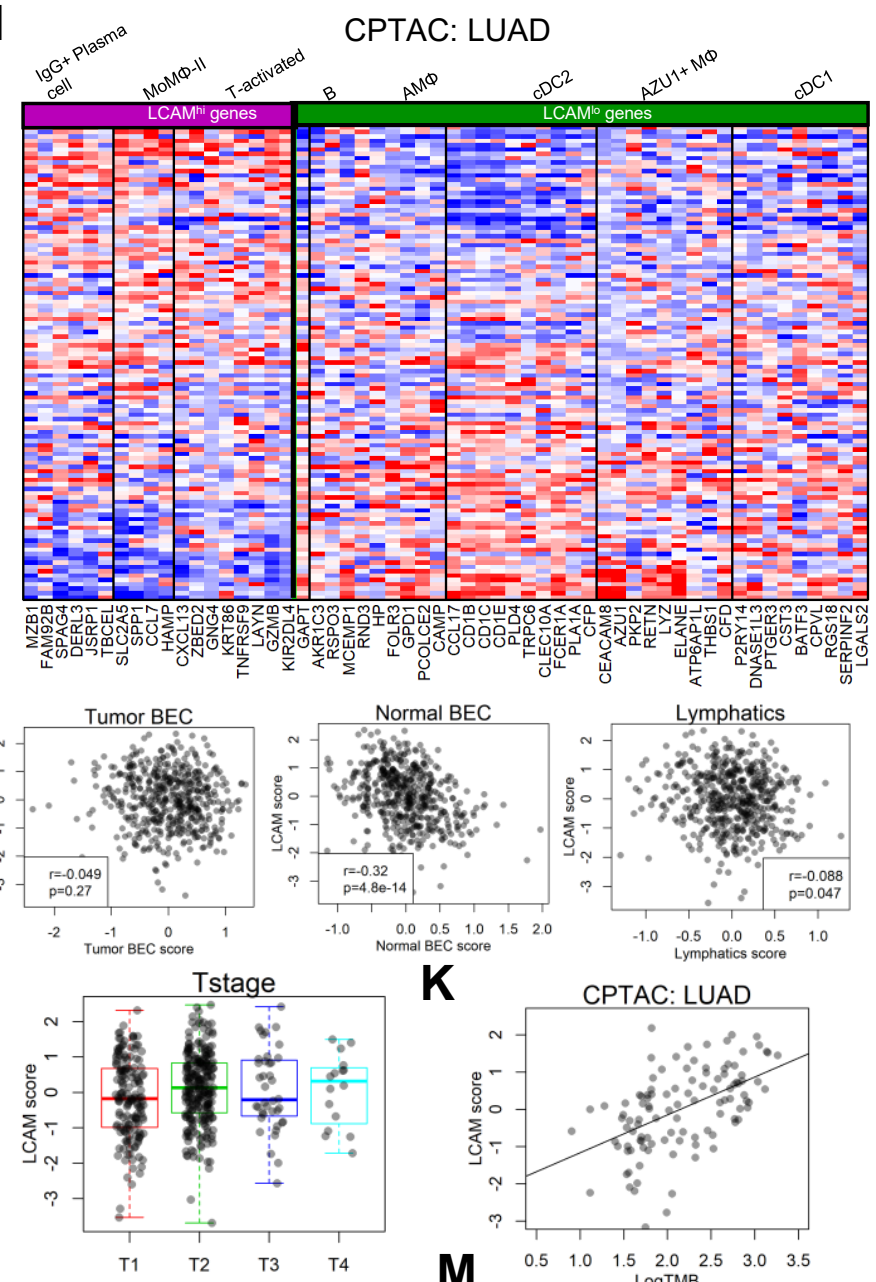

L

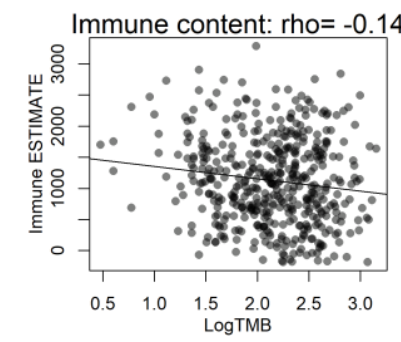

$\mathbf{N}$

Correlation with TMB

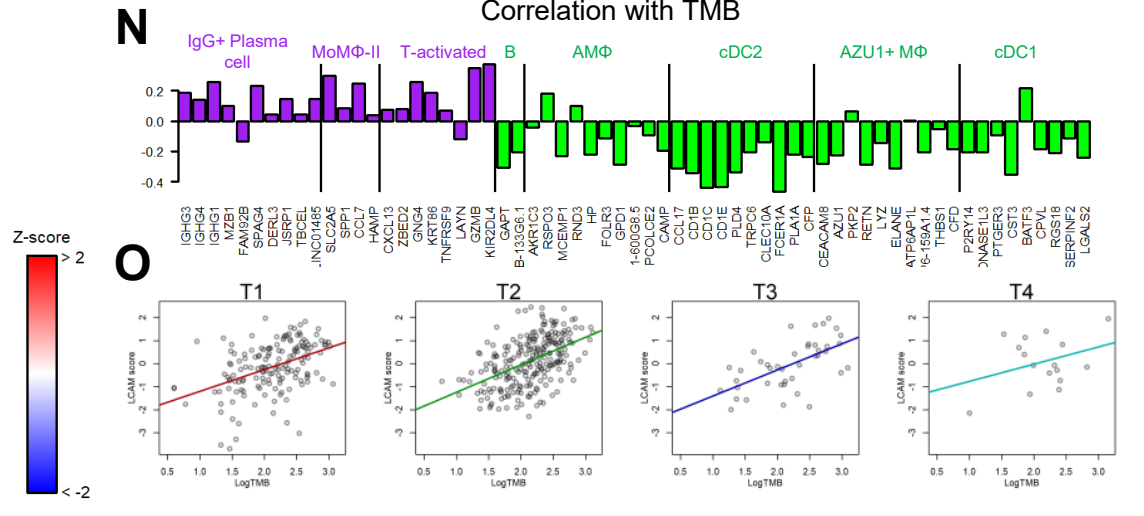

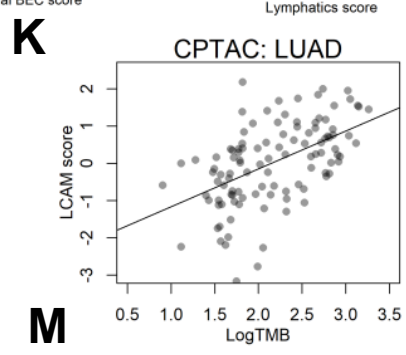

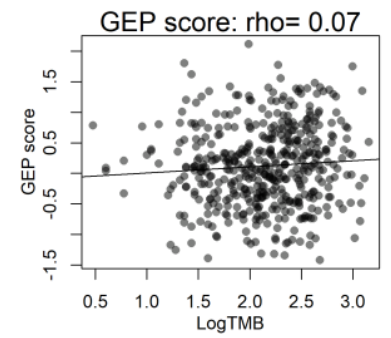

$\mathbf{P}$

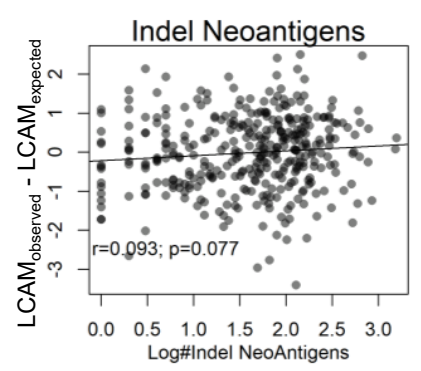

$\mathbf{R}$

Q

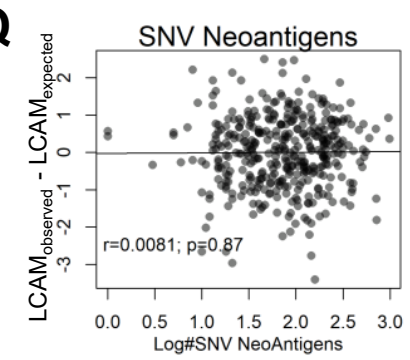

S CPTAC: LUAD

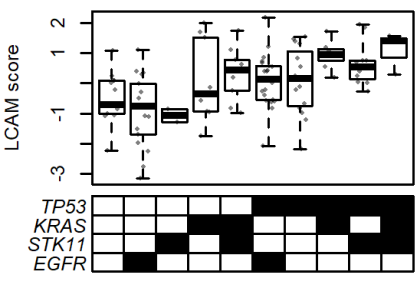

$\mathbf{T}$

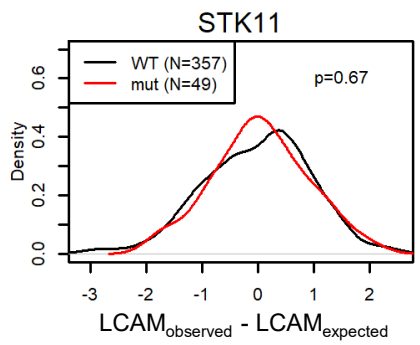

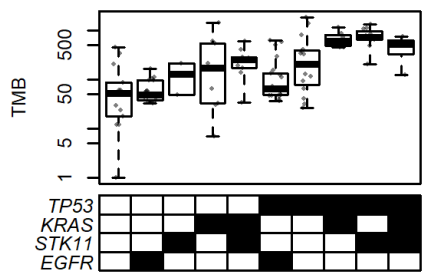

U

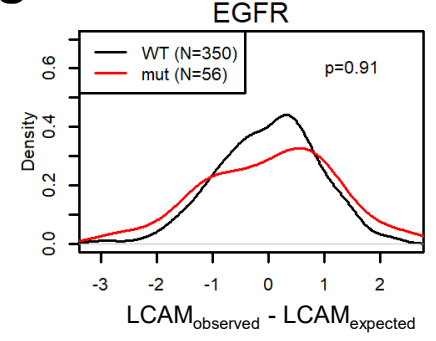

